
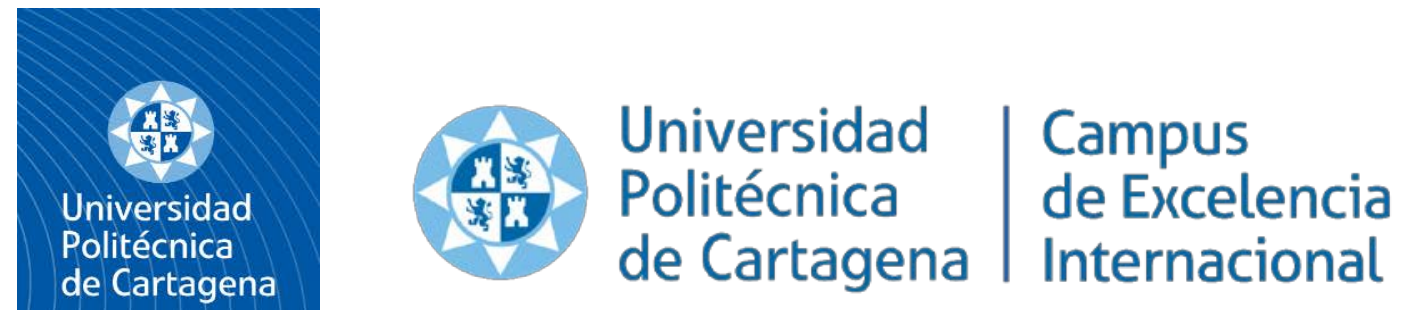

GESTIÓN DEL RIEGO BASADO EN LA VELOCIDAD DE INFILTRACIÓN DEL AGUA EN EL SUELO MEDIANTE LISIMETRÍA DE PESADA

Doctorado en Técnicas Avanzadas en Investigación y Desarrollo Agrario y Alimentario Autor: Laura Ávila Dávila

Directores: Dr. José Miguel Molina Martínez

Dr. Carlos Francisco Bautista Capetillo Dr. Julián González Trinidad Cartagena, 2021 


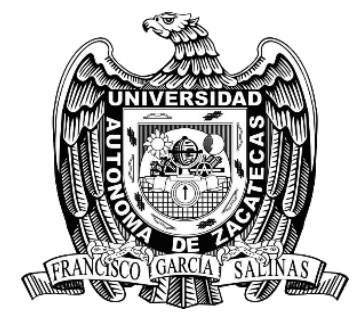

Universidad Autónoma de Zacatecas

"Francisco García Salinas"

Unidad Académica de Ingeniería Eléctrica

Doctorado en Ciencias de la Ingeniería

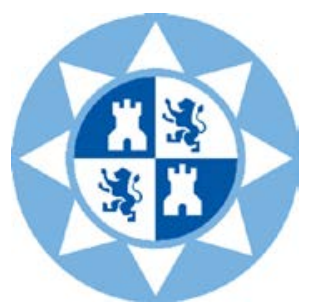

Universidad Politécnica de Cartagena Escuela Técnica Superior de Ingeniería Agronómica

Doctorado en Técnicas Avanzadas en Investigación y Desarrollo Agrario y

Alimentario

\title{
GESTIÓN DEL RIEGO BASADO EN LA VELOCIDAD DE INFILTRACIÓN DEL AGUA EN EL SUELO MEDIANTE LISIMETRÍA DE PESADA
}

\author{
Tesis Doctoral \\ en modalidad de cotutela
}

presentada por

Laura Ávila Dávila

para optar al Grado de Doctora por la Universidad Autónoma de Zacatecas y por la Universidad Politécnica de Cartagena

Director de Tesis en la UAZ

Dr. Carlos Francisco Bautista Capetillo
Director de Tesis en la UPCT Dr. José Miguel Molina Martínez

Codirector de Tesis

Dr. Julián González Trinidad 
Universidad

Politécnica

de Cartagena

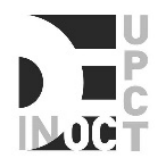

DT-16

\section{CONFORMIDAD DE SOLICITUD DEAUTORIZACIÓN DE DEPÓSITO DE}

TESIS DOCTORAL POR EL/LA DIRECTOR/A DE LA TESIS

$\mathrm{D} / \mathrm{D}^{\mathrm{a}}$. José Miguel Molina Martínez

Director de la Tesis doctoral

Gestión del riego basado en la velocidad de infiltración del agua en el suelo mediante lisimetría de pesada

\section{INFORMA:}

Que la referida Tesis Doctoral, ha sido realizada por D/D . Laura Ávila Dávila , dentro del Programa de Doctorado Técnicas Avanzadas en Investigación y Desarrollo Agrario y Alimentario , dando mi conformidad para que sea presentada ante el Comité de Dirección de la Escuela Internacional de Doctorado para ser autorizado su depósito.

$\checkmark$ Informe positivo sobre el plan de investigación y documento de actividades del doctorando/a emitido por el Director/Tutor (RAPI).

La rama de conocimiento en la que esta tesis ha sido desarrollada es:

$\bigcirc$ Ciencias

Ciencias Sociales y Jurídicas

( Ingeniería y Arquitectura

En Cartagena, a 20/01/2021

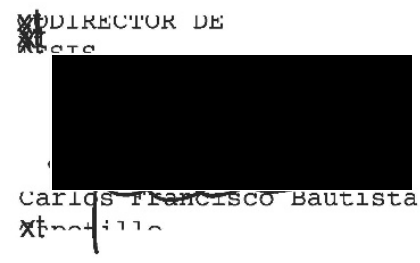

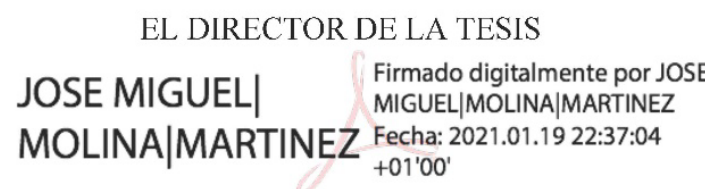

Fdo.:

$x t$

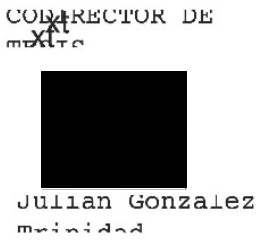

maini And

COMITÉ DE DIRECCIÓN ESCUELA INTERNACIONAL DE DOCTORADOXt 


\section{CONFORMIDAD DE DEPÓSITO DE TESIS DOCTORAL POR LA COMISIÓN ACADÉMICA DEL PROGRAMA}

D/Da. Francisco Artés Hernández Presidente/a de la

Comisión Académica del Programa de Doctorado en Técnicas Avanzadas en Investigación y Desarrollo Agrario y Alimentario.

\section{INFORMA:}

Que la Tesis Doctoral titulada, "Gestión del riego basado en la velocidad de infiltración del agua

en el suelo mediante lisimetría de pesada $"$, ha sido realizada, dentro del

mencionado Programa de Doctorado, por
$\mathrm{D} / \mathrm{D}^{\mathrm{a}}$. Laura Ávila Dávila_ , bajo la dirección y supervisión del Dr./ Dra. José Miguel Molina Martínez

En reunión de la Comisión Académica, visto que en la misma se acreditan los indicios de calidad correspondientes y la autorización del Director/a de la misma, se acordó dar la conformidad, con la finalidad de que sea autorizado su depósito por el Comité de Dirección de la Escuela Internacional de Doctorado.

Evaluación positiva del plan de investigación y documento de actividades por el Presidente de la Comisión Académica del programa (RAPI).

La Rama de conocimiento por la que esta tesis ha sido desarrollada es:

Ciencias

Ciencias Sociales y Jurídicas

- Ingeniería y Arquitectura

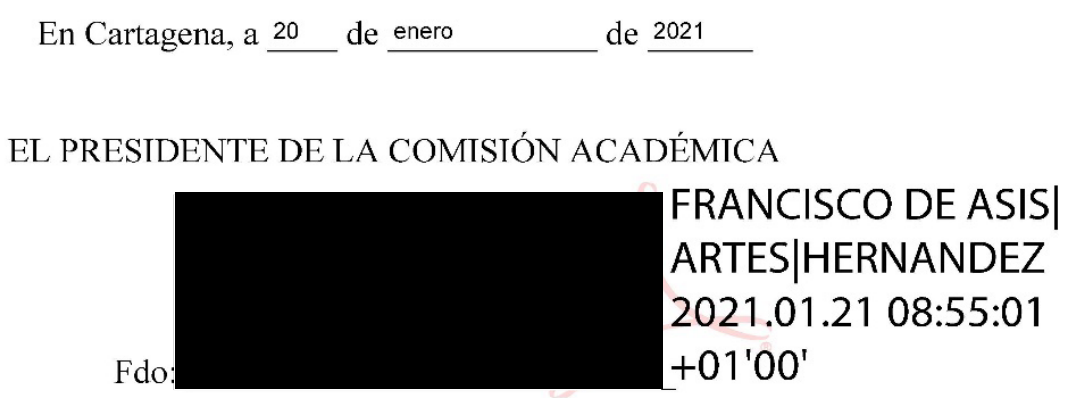

COMITÉ DE DIRECCIÓN ESCUELA INTERNACIONAL DE DOCTORADO 


\section{Agradecimientos}

Esta Tesis Doctoral es un fruto de la dedicación, esfuerzo y paciencia de un grupo de personas que han puesto los medios, conocimientos y tiempo necesarios. En las siguientes líneas expresaré mi agradecimiento a todas aquellas personas que han colaborado en el desarrollo de esta Tesis.

A los Dres. Carlos Francisco Bautista Capetillo y Julián González Trinidad, mis directores de Tesis, por darme la oportunidad de crecer y fortalecer mis conocimientos al permitirme realizar este trabajo bajo su dirección, Por su confianza desde el primer instante, sus consejos y enseñanzas y brindarme los recursos necesarios para lograr la meta.

Al Dr. José Miguel Molina Martínez, director de Tesis, por permitirme realizar mi estancia académica en su grupo de investigación y motivarme para obtener el grado de Doctora por ambas universidades. Por hacerme parte de sus investigaciones y permitirme usarlas para el desarrollo de este trabajo, su confianza puesta sobre mí y sus consejos.

Al Consejo de Nacional de Ciencia y Tecnología (CONACYT) por el apoyo económico durante los tres años del posgrado. 
Al Grupo Operativo GENHIDRO, a la empresa AGROSOLMEN y al Fondo Europeo de Desarrollo Regional (FEDER), cuya financiación mediante el proyecto “Desarrollo e implementación de un equipo de ferticontrol por lisimetría de pesada para uso en agricultura intensiva” con referencia IDI-20190146 han permitido la realización de los trabajos de investigación enmarcados en esta Tesis Doctoral.

A mis amigos, por su cariño y apoyo en toda mi vida académica, que tal vez sin ustedes no hubiera llegado hasta este momento. Por estar siempre al tanto de mis avances y logros, y alegrarse por ellos. Especialmente, por los buenos momentos vividos.

A David, por aparecer en mi vida, por alentarme a conseguir mis propósitos y no rendirme, por creer en mí, por sus consejos y por estar siempre que necesito de su apoyo o ser escuchada. Por los grandes momentos vividos, únicos e irrepetibles, y por ser una de las personas que me quiere ver feliz.

Y finalmente a mi familia, por su amor, apoyo incondicional y confianza en mí. A mis hermanos, por hacer más ameno mis momentos de estrés y desánimo con sus palabras de aliento. A mis padres, por alegrarse y estar orgullosos de mis logros académicos, aunque sin entender muy bien a qué me dedicaba me alentaban a luchar por mis propósitos. A mi mamá Martha, por estar siempre cuando la he necesitado y su cariño. 


\section{Índice}

Resumen

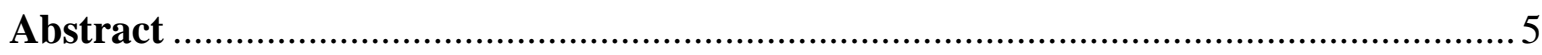

1. Introducción

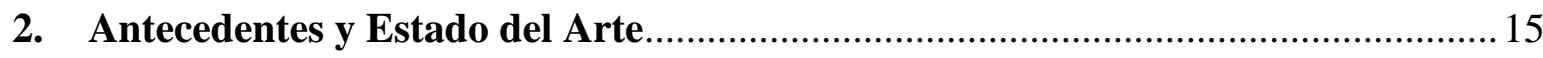

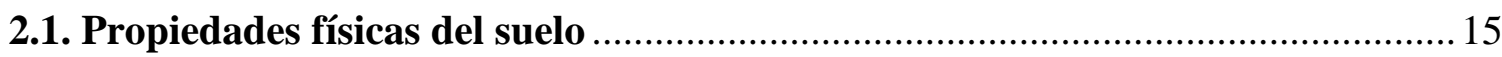

2.2. Propiedades hidrodinámicas del suelo ............................................................... 17

2.2.1. Movimientos del agua en el suelo .................................................................... 17

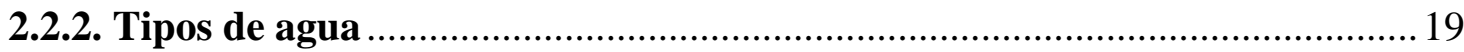

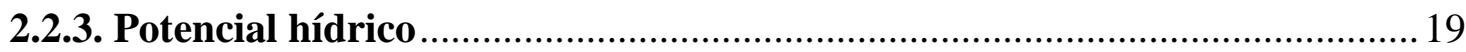

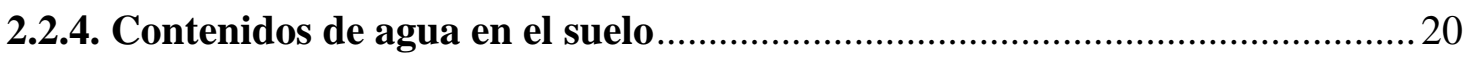

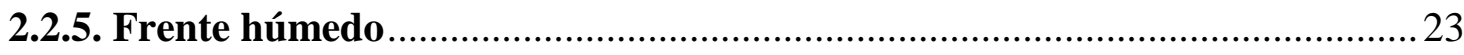

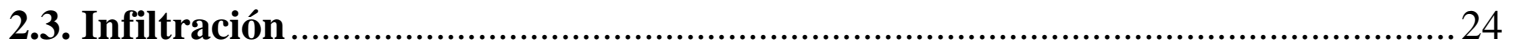

2.4. Evapotranspiración del cultivo ………………………….................................... 32

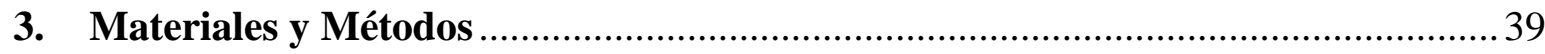

3.1. Determinación de la infiltración del agua del suelo .................................................. 39

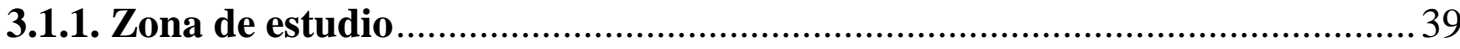

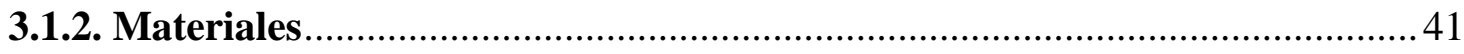

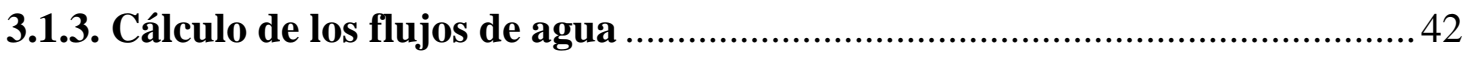

3.1.4. Cálculo de la velocidad de infiltración del agua en el suelo ........................... 44

3.1.5. Estimación del contenido de agua en el suelo …………………………….... 45

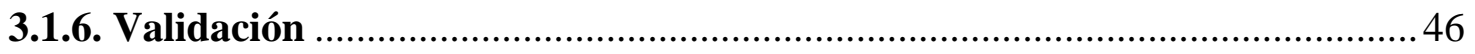

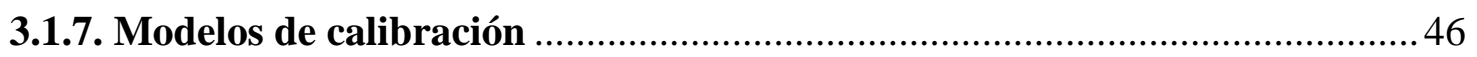

3.2. Evapotranspiración del pimiento y coeficiente de cultivo....................................... 49

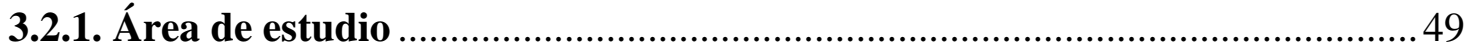


3.2.2. Descripción del lisímetro de pesada compacto ....................................... 49

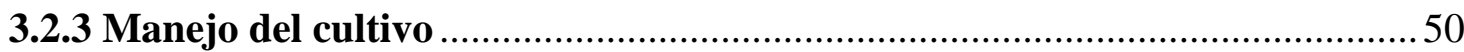

3.2.4. Determinación de la Evapotranspiración y coeficientes de cultivo .............. 51

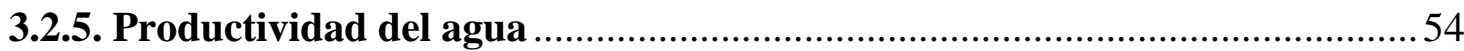

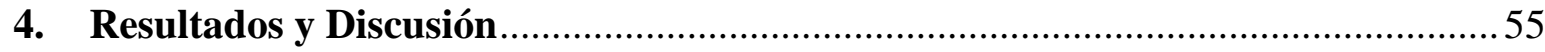

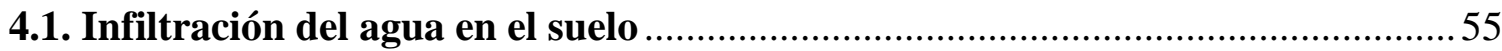

4.1.1. Estimación de la entrada de la lluvia..................................................... 57

4.1.2. Estimación del contenido de agua en el suelo ......................................... 58

4.1.3. Velocidad de infiltración del agua en el suelo ....................................... 60

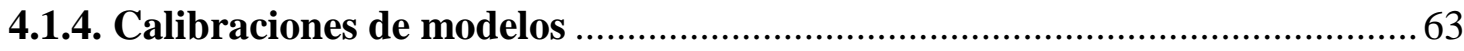

4.2. Evapotranspiración del pimiento y coeficiente de cultivo ................................. 65

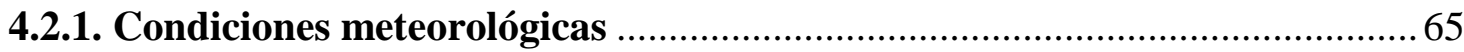

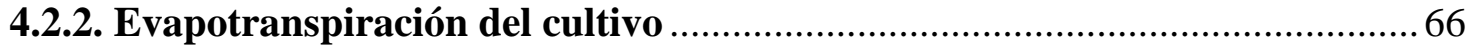

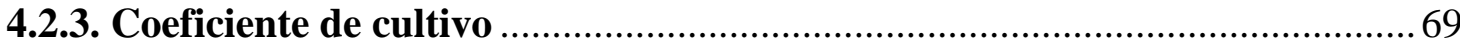

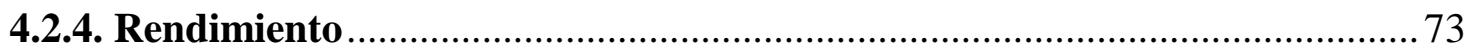

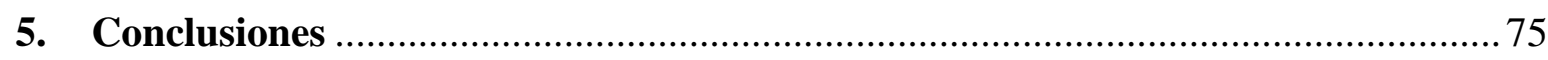

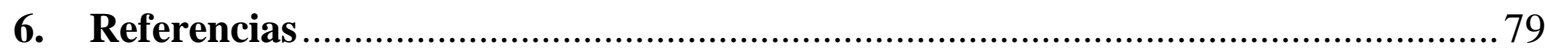

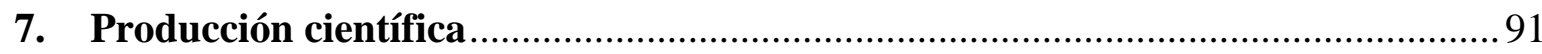

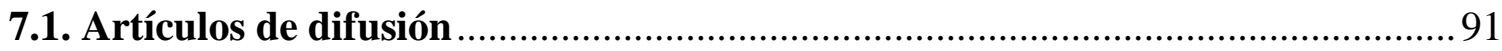

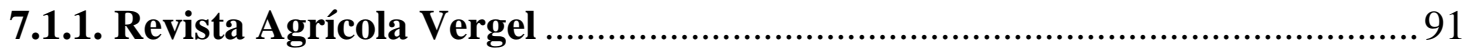

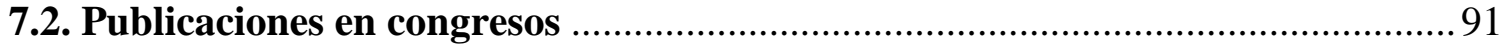

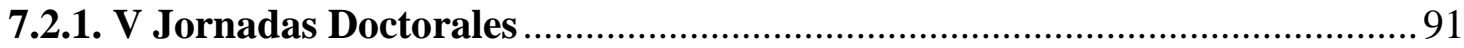

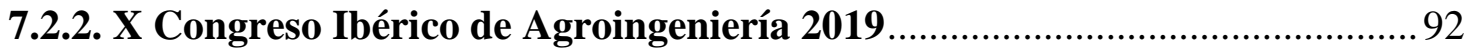

7.2.3. II Symposium Ibérico de Ingeniería Hortícola 2020 ............................... 93

7.2.4. II Congreso de Jóvenes Investigadores en Ciencias Agroalimentarias........ 94 
7.2.5. I Congreso Universitario en Innovación y Sostenibilidad Agroalimentaria

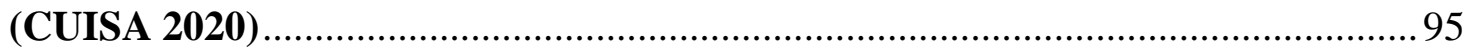

7.3 Artículos en revistas indexadas como coautora durante el doctorado ................95

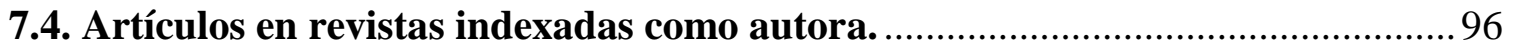

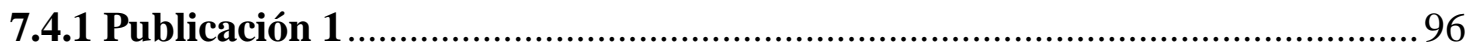

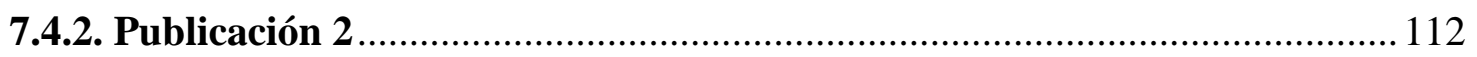

7.5. Índices de impacto de las revistas......................................................... 129

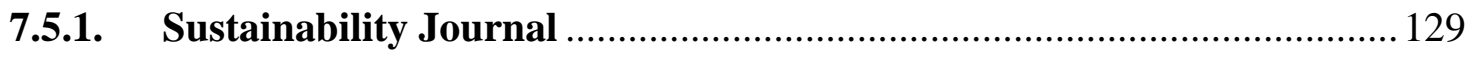

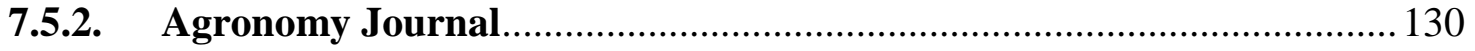





\section{Índice de Figuras}

Figura 1. Sistema trifásico del suelo. 16

Figura 2. Poros de un suelo de textura gruesa (arenoso) y un suelo de textura fina (arcilloso). 16

Figura 3. Representación de los movimientos del agua a través del suelo durante un evento de lluvia. .18

Figura 4. Contenidos de humedad en el suelo. 21

Figura 5. Contenido de agua, potencial hídrico y poros en función al tipo de agua en el suelo. 22

Figura 6. Avance del frente húmedo en diferentes horizontes del suelo: a) textura fina, b) textura gruesa y c) horizonte inferior de textura fina e inferior de textura gruesa. ................23

Figura 7. Experimentación para la ley de Darcy................................................................ 25

Figura 8. Infiltrómetro de doble anillo. ............................................................................. 31

Figura 9. Zona de estudio, finca Las Tiesas en Alicante España. .......................................... 40

Figura 10. Lisímetro de pesada compacto, a) vista general, b) vista detallada y c) movimiento del agua a través de los recipientes........................................................................................ 42

Figura 11. Área de estudio, a) Región de Murcia y b) localización de la parcela experimental.

Figura 12. Vista superior del lisímetro de pesada compacto durante la experimentación. . 50

Figura 13. Variaciones de masa de los dos recipientes del lisímetro de pesada compacto durante el 2017. .56

Figura 14. Contenido de agua en el suelo y variable hidrológicas obtenidas con el lisímetro de pesada compacto, a) Marzo y b) Abril.............................................................................. 58

Figura 15. Diagramas de dispersión de la velocidad de infiltración del agua en el suelo...61

Figura 16. Ajuste de modelos de infiltración a los valores de 1314 de marzo...................... 63

Figura 17. Datos meteorológicos promedio para períodos de 10 días, a) temperatura ambiente, b) humedad relativa, c) velocidad del viento y d) evapotranspiración de referencia ETO. 65

Figura 18. Evapotranspiración diaria del pimiento medido con el lisímetro de pesada (ETCLYS), riego, lluvia y drenaje, a) 2019 y b) 2020. 66 
Figura 19. Correlación entre ETCLYS medido por el lisímetro de pesada y ETCFAO determinado por la metodología de la FAO-56 y entre ETCLYS y ETO. 69

Figura 20. Coeficientes de cultivo del pimiento durante el ciclo de cultivo (valores del lisímetro y la metodología FAO-56).

Figura 21. Ajuste polinómico de los valores del coeficiente de cultivo $(K C)$ obtenidos para 2019 y 2020: a) Ajuste polinómico de segundo orden y b) ajuste polinómico de tercer orden. 


\section{Índice de Tablas}

Tabla 1. Datos de lluvia de los días analizados por los diferentes dispositivos ................. 57

Tabla 2. Balance hídrico de los días involucrados en la determinación de la $\theta F C$............ 59

Tabla 3. Comparación del contenido de agua a capacidad de campo obtenido en el suelo con

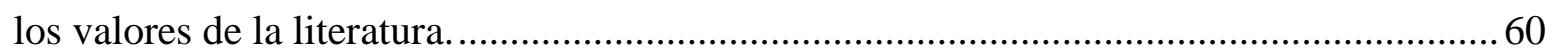

Tabla 4. Valores de la velocidad de infiltración del agua en un suelo franco limoso.........62

Tabla 5. Valores de los parámetros de los modelos de infiltración para el suelo franco limoso.

Tabla 6. Balance hídrico de temporada en milímetros. ................................................67

Tabla 7. Valores de $K C$ obtenidos en este estudio y otros trabajos................................. 73

Tabla 8. Datos de producción de ambas temporadas.................................................. 74 



\section{Resumen}

La agricultura es el mayor consumidor de agua en el mundo, y ante la creciente demanda del uso público e industrial, el uso de eficiente del agua en el riego es una necesidad que va cobrando mayor relevancia. En regiones áridas y semiáridas donde el agua es escasa, tal es el caso del sureste español, la implementación de las tecnologías ha permitido hacer un mejor manejo y gestión del agua. Las herramientas de precisión, como lo son los lisímetros de pesada, proporcionan información en tiempo real, por lo cual, es posible hacer una caracterización y definición del comportamiento de los parámetros involucrados en el movimiento del agua en el suelo, del desarrollo vegetativo del cultivo y el estado hídrico del cultivo.

En esta Tesis, dos enfoques para la medición de la velocidad de infiltración del agua en el suelo fueron propuestos a partir de los valores de masa reportados por los dos recipientes de un lisímetro de pesada, datos obtenidos bajo distintas condiciones de lluvia y diferentes contenidos de humedad en el suelo. Además, la evapotranspiración y los coeficientes de desarrollo vegetativo del pimiento (Capsicum annuum $L$ ) fueron determinados durante la temporada primavera verano de los años 2019 y 2020. Ambas investigaciones realizadas para ayudar en el uso eficiente del agua en la agricultura en climas semiáridos y con escases de agua generalizada. 
Usualmente, la infiltración del agua en el suelo es medida en campo mediante pruebas que requieren el uso de los cilindros concéntricos, sin embargo, estos dispositivos son propensos a errores ya que existe un movimiento lateral del agua bajo el anillo. En las últimas décadas se han desarrollado varias posibilidades para compensar estos errores, que se basan en principios físicos, electrónicos y matemáticos. Así que la primera línea de actuación de esta Tesis fue centrada en el desarrollo de dos enfoques que permitan la determinación de la velocidad de infiltración del agua en un suelo franco limoso, usando los datos de masa reportados por un lisímetro de pesada.

Partiendo del hecho de que con el lisímetro de pesada la determinación de los flujos actuantes en el suelo es más precisa, como se han demostrado con la evapotranspiración y el drenaje. Fue asumido un movimiento vertical descendente del agua en el suelo y con ayuda de la ley de conservación de la masa, 12 eventos de lluvia fueron analizados. Se ha monitoreado el comportamiento del contenido de humedad del suelo y se estableció el contenido a capacidad de campo a partir de los valores del lisímetro de pesada en el que ambos enfoques son basados. La velocidad de infiltración de estos eventos mostró una velocidad variable al comienzo de la lluvia hasta alcanzar un máximo, para descender a una velocidad estable o básica. Esta velocidad de infiltración básica fue de $1.49 \pm 0.36 \mathrm{~mm} \cdot \mathrm{h}^{-1}$, una velocidad dentro del rango de los suelos con texturas finas que reportan una baja capacidad de infiltración. Cuatro modelos empíricos o semiempíricos de infiltración de la literatura fueron calibrados con los valores obtenidos con nuestros enfoques, mostrando un mejor ajuste el modelo de Horton.

La Organización de las Naciones Unidas para la Alimentación y la Agricultura (FAO) ofrece coeficientes normalizados de diferentes cultivos para establecer las necesidades de hídricas de los mismos. Sin embargo, estos coeficientes pueden no representar las condiciones climatológicas, edafológicas, prácticas de cultivo del lugar donde se realiza la siembra. Por lo que, en la segunda línea de actuación de esta Tesis, la evapotranspiración $\left(\mathrm{ET}_{\mathrm{C}_{\mathrm{LYS}}}\right)$ y los coeficientes de cultivo $\left(\mathrm{K}_{\mathrm{C}_{\text {lys }}}\right)$ del pimiento fueron determinados con un lisímetro de pesada compacto para el clima Mediterráneo español, de la temporada primavera-verano, entre los meses de febrero y agosto de los años 2019 y 2020. 
$\mathrm{ET}_{\mathrm{C}_{\mathrm{LYS}}}$ fue determinada a partir de un balance de agua con los valores de masa del lisímetro y los valores de $\mathrm{K}_{\mathrm{C}_{\text {lys }}}$ se determinaron como la relación entre la evapotranspiración del cultivo medida con el lisímetro de pesada y la evapotranspiración de referencia. Los valores medios de $\mathrm{K}_{\mathrm{C}_{\text {lys }}}$ para el pimiento para las etapas inicial, media y final fueron 0.57, 1.06 y 0.80, respectivamente. Se obtuvieron modelos de regresión del coeficiente de cultivo $\left(\mathrm{K}_{\mathrm{C}}\right)$ en función de las unidades térmicas fraccionales, logrando una correlación máxima de $0.67\left(\mathrm{R}^{2}\right)$. En general, los valores de $\mathrm{K}_{\mathrm{C}}$ obtenidos en este trabajo de investigación fueron menores en las etapas inicial y final y mayores en la etapa intermedia, en comparación con los valores de FAO-56 y con los valores de otros países con condiciones semiáridas.

El rendimiento del pimiento también fue evaluado, encontrándose un aumento del 7.72\% en 2019 y un 3.49\% en 2020 en comparación con el rendimiento reportado por el Ministerio de Medio Ambiente y Medio Rural y Marino del Gobierno español en 2019, con pérdidas mínimas de agua por drenaje. Los resultados de trabajo de Tesis pueden ayudar a los agricultores a determinar las necesidades de agua de sus cultivos y a mejorar la eficiencia del agua en lugares semiáridos con condiciones similares a las del estudio. 



\begin{abstract}
Agriculture is the biggest consumer of the global water supplies, competing for the resource with the growing demand for public and industrial use. It is essential to implement technologies that help the better water management in irrigation; this gained relevance in arid and semi-arid regions where water is scarce, such as the southeast of Spain. Precision tools, such as weighing lysimeters, provide real time information for the characterization and definition of the behavior of the parameters involved in the vegetative development, the water status of crops and water movement in the soil, making efficient use of the vital liquid.
\end{abstract}

In this Thesis, two approaches were proposed to measure the water infiltration rate of the soil using the mass values reported by the two lysimeter vessels under different rain conditions and different soil moisture contents. In addition, the evapotranspiration and vegetative development coefficients of bell pepper (Capsicum annuum $L$ ) were determined during the spring-summer season of 2019 and 2020, Both investigations were conducted in the southeast of Spain in the regions of Murcia and Albacete, to contribute to the water efficiency in agriculture in semi-arid climates and widespread shortages of water.

Usually, the infiltration estimation is made by tests with the concentric cylinders, which are prone to errors due to the lateral movement under the ring. Several possibilities have been developed over the last decades to compensate these errors, which are based on 
physical, electronic and mathematical principles. So, the first action line of this Thesis was focused on the development of two approaches to determine the infiltration rate/speed in a silt loam soil by means mass values reported by a weighing lysimeter. Because with the lysimeter it is possible to determine acting soil flows very precisely; then with the help of mass conservation and assuming a downward vertical movement, 12 rain events were analyzed.

In addition, it was possible to monitor the behavior of soil moisture and to establish the content at field capacity from the values of the weighing lysimeter, from which both approaches are based. The infiltration rate of these events showed a variable rate at the beginning of the rainfall until reaching a maximum, to descend to a stable or basic rate. This basic infiltration rate was of $1.49 \pm 0.36 \mathrm{~mm} \cdot \mathrm{h}^{-1}$, this is because soils with fine textures have reported low infiltration capacity. Four empirical or semi-empirical models of infiltration were calibrated with the values obtained with our approaches, showing a better fit with the Horton's model.

The Food and Agriculture Organization of the United Nations (FAO) offers standardized crop coefficients to establish the water requirement of crops. However, these coefficients can change due to different conditions, such as climatic variations and cultivation practices. So, in the second action line of this Thesis, the actual evapotranspiration $\left(\mathrm{ET}_{\mathrm{C}_{\mathrm{lys}}}\right)$ and crop coefficients $\left(\mathrm{K}_{\mathrm{Clys}_{\mathrm{l}}}\right)$ of bell pepper were determined with a compact removable weighing lysimeter between February and August for two crop seasons 2019 and 2020 for the Spanish Mediterranean.

$\mathrm{ET}_{\mathrm{C}_{\text {lys }}}$ was determined from the water balance, and the $\mathrm{K}_{\mathrm{C}_{\text {lys }}}$ values were determined as the ratio of the actual evapotranspiration, measured on the removable weighing lysimeter, and the reference evapotranspiration. The average values of $\mathrm{K}_{\mathrm{C}_{\mathrm{lys}}}$ for the bell pepper for the initial, middle, and final stages were $0.57,1.06$, and 0.80 , respectively. $\mathrm{K}_{\mathrm{C}}$ regression models were obtained as a function of the fraction thermal units, achieving a maximum correlation of $0.67\left(\mathrm{R}^{2}\right)$. In general, the $\mathrm{K}_{\mathrm{C}}$ values obtained in this research work were lower in the initial 
and end stages and larger in the middle stage compared to the FAO-56 values and to values in other countries with semiarid conditions.

The bell pepper yield increased by 7.72\% in 2019 and by 3.49\% in 2020 compared to the yield reported by the Ministry of the Environment and Rural and Marine Areas of the Spanish Government in 2019 and with minimal water loss through drainage. The results in this work can help farmers to determine the crop water requirements and to improve system efficiency in semiarid locations with conditions like those of the study. 



\section{Introducción}

El 71\% de la superficie del planeta está cubierto por agua, de la cual el $97.5 \%$ es salada y está retenida en los océanos, mientras que el 2.5\% es agua fresca o dulce. El 68.7\% del agua dulce está congelada en glaciares y casquetes polares, el 30.1\% es agua subterránea y el 1.2\% está en la superficie en forma de lagos, ríos, vapor de aire, humedad en el suelo, pantanos, seres vivos e hielo terrestre (USGS, 1993).

El sector agrícola es el mayor usuario de agua dulce en el mundo, consumiendo cerca 85\% del total (Vico and Porporato, 2010). La agricultura compite con otros sectores por el vital líquido, tales como la industria, la minería, la demanda urbana, entre otros, haciendo que aumente la escases de agua para el sector agrícola (Sanchéz-Bravo et al., 2020). La agricultura de regadío es de vital importancia para la población mundial ya que produce el $40 \%$ de los alimentos en el mundo, en tan sólo el 18\% del total del tierras cultivas alrededor del mundo (García-Tejero et al., 2011).

La distribución desigual del agua y el impacto del cambio climático, causante de temperaturas elevadas y bajos niveles de precipitación, provocan mayor demanda hídrica de los cultivos y añaden incertidumbre al garantizar el abastecimiento de agua a la agricultura (Evans and Sadler, 2008). Esta problemática es reflejada en las regiones áridas y semiáridas, donde las fuentes de abastecimiento se ven reducidas a medida que el cambio climático va 
aumentando (Parra et al., 2020). En consecuencia, estas regiones demandan estrategias para mejorar la eficiencia del uso del vital líquido para obtener cultivos sostenibles y económicamente rentables, este es el caso del sureste de España (Fernández-Pacheco et al., 2014; Navarro-Hellín et al., 2016).

La eficiencia de la aplicación del agua en el riego es baja, alrededor del mundo se estima cerca del 40\%, significando que el 60\% del agua extraída de las fuentes de abastecimiento es desperdiciada. Al mejorar la eficiencia de aplicación coadyuvará a mejorar los beneficios económicos y ambientales (Ooi et al., 2010). Entre los diferentes métodos de riego que logran hacer un uso eficiente del agua es el localizado (Sandhu et al., 2019), su eficiencia de aplicación van de 70 a 95\% suponiendo un sistema de riego en buenas condiciones y bien diseñado (Pereira et al., 2010); sin embargo, si este no se cumple, el sistema derrochará agua debido a que la tasa o el volumen de aplicación del riego no es el adecuado, el sistema es defectuoso o ambos.

Para hacer un uso eficiente del recurso hídrico es necesario el entendimiento de la actuación de los flujos de agua en el sistema suelo-planta-atmosfera. A partir del balance de esos flujos es posible conocer los requerimientos hídricos de cualquier cultivo. Los flujos considerados como entrantes a dicho sistema son el riego y la lluvia; la evapotranspiración, el drenaje más allá de la zona de raíces y los escurrimientos superficiales son considerados como los flujos de salida o pérdidas de agua (Jensen, 2007).

Los requerimientos o necesidades hídricas del cultivos son definidos por la evapotranspiración, este parámetro incluye características genéticas del cultivo y condiciones climáticas a los que se está siendo sometidos (Allen et al., 1998). Antes de satisfacer dicho requerimiento hídrico es necesario conocer la capacidad que tiene el suelo en aceptar y almacenar el agua entrante, esto para no aplicar de manera arbitraria el agua y causar escurrimientos superficiales no deseados. Esto ayudará a controlar la disponibilidad de agua dentro de la zona de raíz, en orden para mejorar la calidad y productividad de los cultivos, y así, establecer una programación de los requerimientos hídricos del cultivo con una tasa de aplicación del riego adecuada. 
En este contexto, la velocidad de infiltración indicará la cantidad o el volumen de agua que entra en determinado tiempo (Kirkham, 2010). La infiltración del agua en el suelo varia en espacio y tiempo, debido a la textura del suelo, prácticas culturales, porosidad, densidad aparente, variaciones del clima, compactación, contenido de humedad inicial, entre otras (Mattar et al., 2015; Yuan et al. 2019). Cuando la intensidad de lluvia o la tasa de riego excede la capacidad de infiltración del suelo se producen encharcamientos y escurrimientos superficiales, causando bajas eficiencias de aplicación del riego y erosión del suelo por el arrastre de las partículas sólidas con el agua (Ali, 2010; Arriaga et al., 2010).

Existen modelos matemáticos que describen el movimiento del agua en el suelo a partir de variables que gobiernan e influyen en el proceso de infiltración que requieren de experimentación complicada y detallada para describirlo. A largo de los años estos modelos han sido simplificados a modelos empíricos o semiémpiricos resultantes de la experimentación en las diferentes texturas de suelo. Los dispositivos que usualmente han sido usado para medir in situ la velocidad de infiltración del agua debido a su simplicidad y fácil operación en una dimensión son los infiltrómetros (Ali, 2010; Mahmood and Latif, 2005; Teófilo-Salvador and Morales-Reyes, 2018).

El volumen infiltrado en estos dispositivos es medido con dos enfoques, volumen de agua necesario para mantener un nivel constante de encharcamiento en el anillo interior o volumen obtenido por la disminución del nivel de agua del anillo interior (Arriaga et al., 2010; Fatehnia et al., 2016). Sin embargo, los datos obtenidos con estos dispositivos pueden no representar un movimiento vertical, debido a que pueden presentarse flujos horizontales de agua debajo de los anillos y que representaría errores de medición; dichos anillos son de acero y crean una compactación en un suelo húmedo o destrucción de la estructura de un suelo seco ya que son instalados usando la fuerza (Bagarello and Sgroi, 2004).

Una vez caracterizado y entendido el comportamiento del agua en el suelo, una forma sencilla de lograr la programación de los riegos es mediante la utilización de manuales de riego. Estos ofrecen valores estándar de los coeficientes de desarrollo de diferentes cultivos y velocidades de infiltración de diferentes texturas de suelos. Sin embargo, al usarlos no se estaría representando las condiciones climáticas del lugar en donde se realiza la plantación, 
ya que dichos valores fueron calibrados en condiciones distintas, y esto puede causar un exceso en la aplicación del agua e ir arrastrando un sesgo en la planeación. Además, éstas metodologías no cuenta con una supervisión continua en el tiempo, y no es posible detectar alguna incidencia (Elaydi, 2017). Mediante el uso de tecnologías es posible medir los parámetros que intervienen en el riego, obteniendo información previa y haciendo un monitoreo continuo en el tiempo de estos parámetros que ayudarán en el diseño y corrección de la aplicación del agua (Katyara et al., 2017).

Entre las tecnologías que ayudan en la planeación del riego están los sensores electrónicos que monitorizan y miden las variables in situ presentes en el clima, el suelo y planta, teniendo en cuenta de ante mano cuáles variables serán las utilizadas en la toma de decisión del cuándo y cuánto regar, y así saber qué tipo de sensor utilizar (Pereira et al., 2010). En este contexto, los lisímetros de pesada han sido ampliamente usados para establecer y calibrar modelos matemáticos referentes al riego, ya que miden de manera directa las pérdidas de agua mediante la evapotranspiración o drenaje y los flujos entrantes de agua como la lluvia o el riego (Bello and Van Rensburg, 2017; Hannes et al., 2015). Por lo que muestran ser una herramienta factible y precisa de medir de forma directa la infiltración del agua en el suelo.

A partir de lo anteriormente descrito, se ha planteado la siguiente hipótesis, con la utilización de un lisímetro de pesada es posible hacer la gestión del riego mediante la caracterizar de la capacidad de infiltración de un suelo y la determinación de la evapotranspiración de cultivo, por su alta precisión de medición, y sus buenos resultados en la determinación de los flujos de agua actuantes en el suelo.

Para demostrar dicha hipótesis, el objetivo general de esta Tesis Doctoral fue hacer una gestión del riego y optimización del recurso hídrico en zonas con limitaciones de agua, como el sureste español, a través del desarrollo y aplicación de enfoques matemáticos que describan la velocidad de infiltración del agua en el suelo en función del contenido de humedad de un suelo franco-limoso y la determinación de la evapotranspiración y coeficientes de desarrollo del pimiento (Capsicum annuum L.) usando los datos de masa de un lisímetro de pesada. 
Para lograr el objetivo general fueron planteados los siguientes objetivos específicos:

1. Desarrollar dos enfoques para la determinación de la velocidad de infiltración del agua cuando el contenido de humedad en el suelo está por encima y por debajo de la capacidad de campo a partir de un balance hídrico de los datos de masa del lisímetro de pesada.

2. Establecer los contenidos de humedad del suelo franco-limoso a lo largo del periodo de tiempo analizado con los datos del lisímetro de pesada, estableciendo principalmente el contenido de agua a capacidad de campo del suelo.

3. Aplicar los enfoques desarrollados de la velocidad de infiltración en un suelo francolimoso durante lluvias inestables.

4. Calibrar los modelos de infiltración de Kostiakov, Kostiakov Modificado, Horton y Philip con los datos obtenidos en los dos enfoques de velocidad de infiltración determinados en el objetivo anterior.

5. Medir la evapotranspiración y determinar los coeficientes de desarrollo del pimiento durante las dos temporadas de plantación del 2019 y 2020 con los datos del lisímetro de pesada, adaptados a las condiciones climáticas del sureste español y técnicas de cultivo propias.

6. Determinar la evapotranspiración del pimiento usando la metodología y valores de la FAO para comparar los resultados obtenidos con el lisímetro de pesada.

7. Determinar las unidades térmicas fraccionales para obtener modelos del coeficiente de desarrollo del cultivo.

8. Evaluar el rendimiento del pimiento durante las temporadas del 2019 y 2020 mediante indicadores de productividad de uso del agua y el valor promedio de producción en la región de Murcia. 



\section{Antecedentes y Estado del Arte}

Se hará una breve definición de los principales conceptos que intervienen en la relación agua-suelo-planta y su estudio a lo largo de los años, para entender el movimiento del agua en el suelo, el proceso de infiltración, y la determinación del requerimiento hídrico del cultivo.

\subsection{Propiedades físicas del suelo}

El suelo es un sistema trifásico poroso (Fig. 1) que consta de una fase sólida, formada por minerales de distintos tamaños y materia orgánica, una fase liquida representada por el agua y una fase gaseosa constituida por aire u otros gases (Ali, 2010). La suma de la masa del agua $\left(\mathrm{M}_{\mathrm{A}}\right)$ y de la masa las partículas sólidas $\left(\mathrm{M}_{\mathrm{S}}\right)$ representan la masa total del suelo $\left(\mathrm{M}_{\mathrm{T}}\right)$, ya que la masa del aire ( $\mathrm{M}_{\mathrm{aire}}$ ) es considerada despreciable. En cambio, el volumen total del suelo $\left(\mathrm{V}_{\mathrm{T}}\right)$ es igual a la suma de los volúmenes de las tres fases del suelo; el volumen de vacíos $\left(\mathrm{V}_{\mathrm{V}}\right)$ representa la suma del volumen de aire $\left(\mathrm{V}_{\text {aire }}\right)$ y el volumen de agua ( $\left.\mathrm{V}_{\mathrm{agua}}\right)$, todo esto es reflejado en la Fig. 1. 


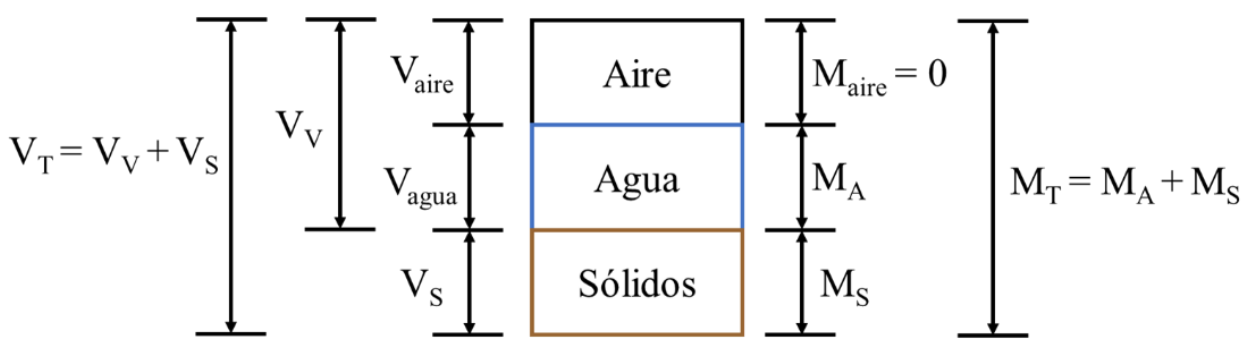

Figura 1. Sistema trifásico del suelo.

La textura o granulometría del suelo indica el contenido de las partículas sólidas en función a su diámetro y se definen tres clases: las partículas gruesas con diámetro entre $2 \mathrm{~mm}$ y $50 \mu \mathrm{m}$ son denominadas arenas, diámetros de partículas de $50 \mu \mathrm{m}$ a $2 \mu \mathrm{m}$ son limos y menores de $2 \mu \mathrm{m}$ son arcillas (Staff, 1951). El tamaño de las partículas sólidas define el tamaño y el número de poros o vacíos del suelo. Un suelo arcilloso cuenta con gran cantidad de espacios porosos, en cambio en un suelo arenoso tiene menos huecos, debido a que sus partículas sólidas son más grandes y ocupan mayor espacio, como se aprecia en la Fig. 2 (Tarjuelo Martín-Benito, 1991).
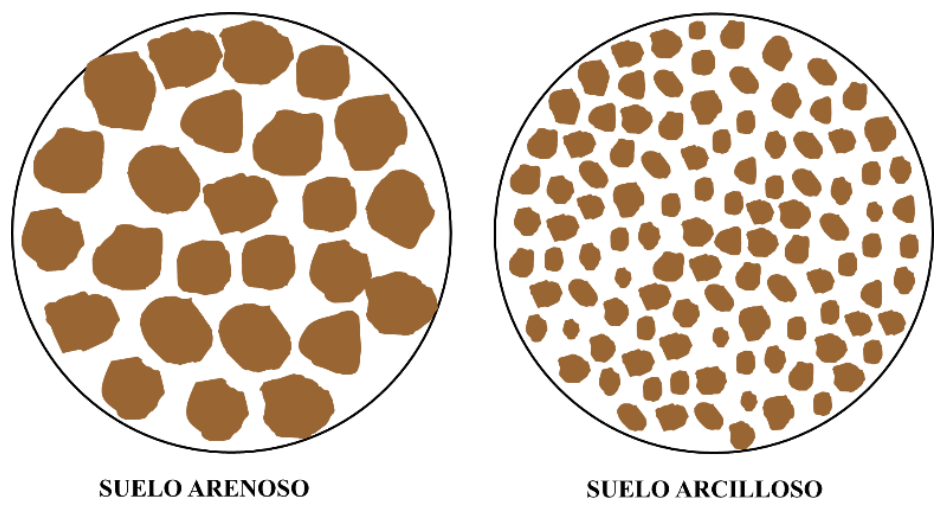

Figura 2. Poros de un suelo de textura gruesa (arenoso) y un suelo de textura fina (arcilloso).

Por ende, la porosidad de un suelo (P) es medida como el cociente entre el volumen de vacíos $\left(\mathrm{V}_{\mathrm{V}}\right)$ y el volumen total que ocupa el suelo $\left(\mathrm{V}_{\mathrm{T}}\right)$; ésta también puede ser estimada a partir de la densidad aparente $\left(\rho_{a}\right)$ y real del suelo $\left(\rho_{r}\right)$. La densidad aparente es definida como la relación entre la masa del suelo secado a estufa $\left(\mathrm{M}_{\mathrm{S}}\right)$ y el $\mathrm{V}_{\mathrm{T}}$, y para obtener la densidad real de un suelo se tiene que dividir la Ms sobre el volumen que ocupa los sólidos $\left(V_{S}\right)$, ésta última densidad tiene un valor promedio de 2.65 a $2.7 \mathrm{~g} \cdot \mathrm{cm}^{-3}$ en partículas 
minerales como las del suelo (Koorevaar et al., 1999). Todo lo antes señalado puede ser observado en la siguiente Ecuación 1 y teniendo en cuenta la Fig. 1:

$$
\mathrm{P}(\%)=\frac{\mathrm{V}_{\mathrm{v}}}{\mathrm{V}_{\mathrm{T}}}=\frac{\mathrm{V}_{\mathrm{T}}-\mathrm{V}_{\mathrm{S}}}{\mathrm{V}_{\mathrm{T}}}=1-\frac{\mathrm{V}_{\mathrm{s}}}{\mathrm{V}_{\mathrm{T}}}=1-\frac{\mathrm{V}_{\mathrm{s}} \times \mathrm{M}_{\mathrm{S}}}{\mathrm{M}_{\mathrm{S}} \times \mathrm{V}_{\mathrm{T}}}=\left(1-\frac{\rho_{\mathrm{a}}}{\rho_{\mathrm{r}}}\right) \cdot 100
$$

Una alta densidad aparente indica un suelo con porosidad pequeña y por lo tanto compactado; la compactación afecta a la aeración, permeabilidad, velocidad de infiltración del agua y drenaje del suelo, además, dificulta el desarrollo de la raíces y la emergencia de la semilla o plántula (Koorevaar et al., 1999).

\subsection{Propiedades hidrodinámicas del suelo}

\subsubsection{Movimientos del agua en el suelo}

Los movimientos de agua a través del espacio poroso en el suelo pueden clasificarse en función en su dirección (Duchaufour, 1998; Plaster, 2013; Wang et al., 2017):

i. Escorrentía superficial, se le domina al agua que circula paralelamente a la superficie del suelo arrastrando las partículas finas de éste.

ii. Movimientos descendentes, es el agua que se mueve de forma descendente a través del suelo debido a la fuerza de gravedad. La velocidad del agua dentro del suelo es rápida en las primeras horas después de la lluvia, ya que el agua primero circula entre los grandes poros; el agua nunca se detiene por lo que seguirá moviéndose lentamente hasta alcanzar la capa permeable del suelo alimentando a las corrientes subterráneas.

Es su paso, el agua va quedando retenida en los poros medianos y pequeños por acción de la capilaridad; es decir, mediante las fuerzas de adhesión y cohesión que son lo suficientemente elevadas para oponerse a la gravedad, las partículas sólidas van creando una película fina de agua alrededor de cada una de ellas y meniscos de agua entre ellas. 
La fuerza de adhesión es la fuerza de atracción ente las moléculas de agua y la partícula del suelo; una vez que la partícula del suelo ya fue recubierta por las moléculas del agua las fuerzas de cohesión comienzan a actuar, es decir las moléculas de agua empieza atraerse entre sí ya que no pueden adherirse a las partículas sólidas. Por lo que únicamente, el agua presente en los meniscos puede ser absorbida por las raíces de la planta.

iii. Movimientos ascendentes, son las pérdidas de agua a través de la evaporación o evapotranspiración en periodos secos donde no hay ingresos de agua en el suelo, por lo que, si el suelo es cercando al manto freático o alguna corriente subterránea tratará de compensar estas pérdidas con agua subterránea a través del efecto de la capilaridad, a esto se le denomina acenso capilar.

En la Fig. 3 se representa el comportamiento del agua de un evento de lluvia dentro del suelo, basado en los tres movimientos anteriormente descritos.

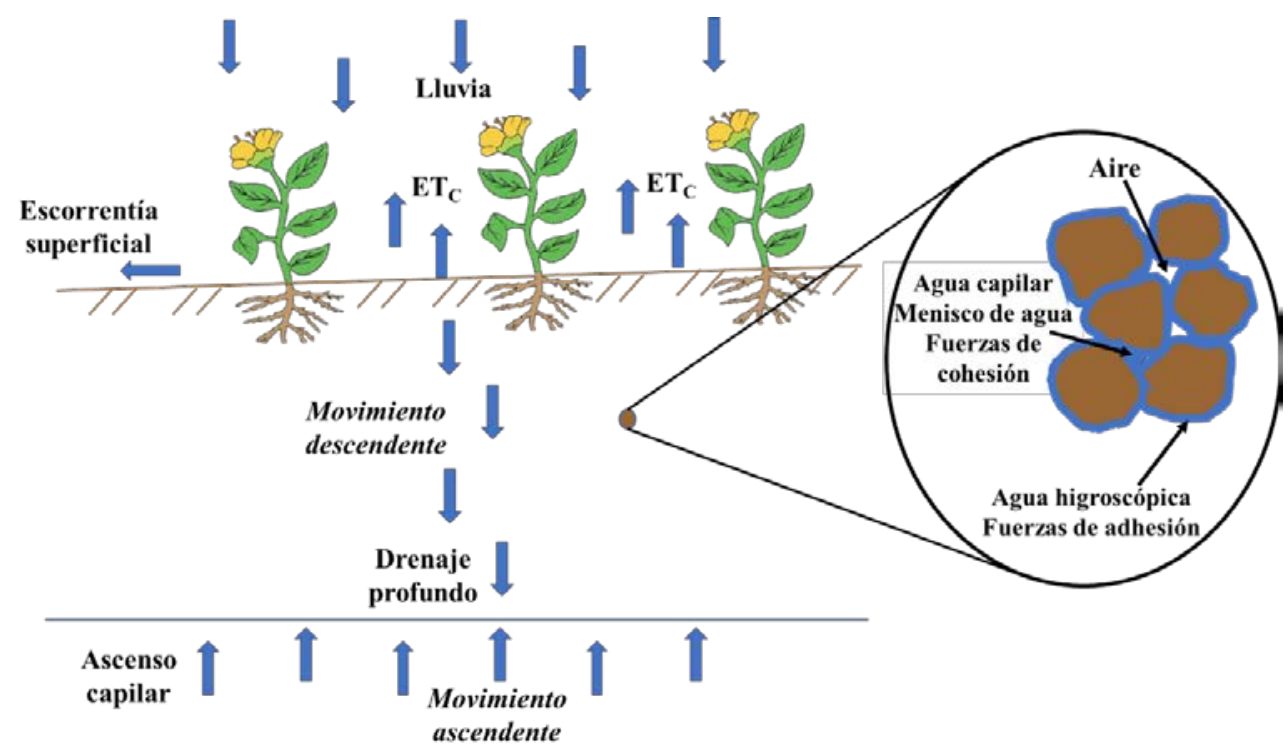

Figura 3. Representación de los movimientos del agua a través del suelo durante un evento de lluvia. 


\subsubsection{Tipos de agua}

A partir de la descripción del movimiento descendiente del agua en el suelo, el agua en el suelo puede agruparse en tres grupos en función a su unión de la partícula del suelo y disponibilidad para las plantas, (Tarjuelo Martín-Benito, 1991), los cuales son:

i. Agua gravitacional, es el agua que ocupa brevemente los grandes poros y que es drenada por la acción de la fuerza de la gravedad.

ii. Agua capilar, es el agua que logra vencer la fuerza de gravedad y es retenida entre las partículas sólidas del suelo, formando un menisco de agua disponible para las plantas, ver Fig.3.

iii. Agua higroscópica, es el agua que está fuertemente ligada a las partículas del suelo y que no puede ser usada por las plantas, ver Fig.3.

\subsubsection{Potencial hídrico}

La tendencia del movimiento descendente del agua desde la superficie del suelo a través de las partículas sólidas, las raíces, macroorganismos, semillas, entre otros, es medida mediante el potencial hídrico del suelo (Foth, 1990). El potencial hídrico ( $\Psi$ ) mide la energía necesaria para que las moléculas de agua realicen el trabajo del movimiento descendente de más a menos potencial (Duchaufour, 1998). El $\Psi$ es el resultado de las contribuciones individuales de los diferentes niveles de energía del agua en el suelo y es expresado como la suma del potencial de gravedad $\left(\Psi_{\mathrm{g}}\right)$, el potencial mátrico $\left(\Psi_{\mathrm{m}}\right)$ y el potencial osmótico $\left(\Psi_{\mathrm{o}}\right)$. Usualmente, la unidad de los potenciales es el bar. 1 bar equivale a $0.1 \mathrm{MPa}, 100 \mathrm{kPa}$ o $3 \mathrm{pF}$ en escala logarítmica (Plaster, 2013).

El $\Psi_{\mathrm{g}}$ es el movimiento que gobierna la infiltración del agua de lluvia, definido como el movimiento desde elevaciones altas a bajas, este potencial tiene un valor positivo y es el primer movimiento en un suelo saturado. El $\Psi_{\mathrm{m}}$ es el resultante de la atracción de agua con 
las partículas del suelo, su valor es negativo y está presente en un suelo insaturado. Por último, el $\Psi_{0}$ se presenta únicamente en suelos con alto contenido en sales, las moléculas de agua son atraídas por los iones de sal, este es el más bajo de los potenciales y tiene un valor negativo. En la mayoría de los suelos, el potencial hídrico sólo está dado por la suma de $\Psi_{\mathrm{g}}$ y $\Psi_{\mathrm{m}}$, representado en la Ecuación 2 (Duchaufour, 1998; Plaster, 2013):

$$
\Psi=\Psi_{\mathrm{g}}+\Psi_{\mathrm{m}}
$$

Cuanto más bajo es el potencial de agua del suelo, el agua es atraída más estrechamente a las partículas del suelo y puede moverse con menos libertad. Usualmente, el valor del potencial hídrico es negativo, porque el valor mayor representa el $\Psi_{\mathrm{m}}$. Por lo tanto, mientras mayor sea el valor absoluto del número negativo, menor será el potencial hídrico. Cuando se habla de un valor potencial hídrico alto, indica que el agua se mantiene libre, se mueve fácilmente y es disponible para las plantas. El agua se mueve de un alto potencial, es decir de un suelo húmedo, a un potencial bajo donde el suelo está seco. Este proceso permite la infiltración, en el cual el agua se mueve fácilmente hasta un estado de bajo potencial. Cuando la lluvia cae a un suelo seco, el agua tiene un alto potencial por lo que tiene un gran movimiento en el suelo (Plaster, 2013).

\subsubsection{Contenidos de agua en el suelo}

A partir de los tipos de agua y de los potenciales definidos anteriormente, tres contenidos de agua o humedad en el suelo son definidos a partir de su disponibilidad para las plantas y retención en el suelo (ver Fig. 4):

i. Contenido de agua a saturación $\left(\theta_{S}\right)$, este contenido de humedad es alcanzado cuando todos los espacios vacíos del suelo se encuentran llenos de agua; en este nivel, el suelo ya no es capaz de aceptar más agua por lo que comienza a desplazar o drenar agua por la parte inferior durante 24 a 48 horas hasta alcanzar un estado de estabilidad. El agua también comienza a almacenarse en la parte superficial, causando el denominado escurrimiento superficial. Un suelo saturado no permite 
la respiración de la planta por los bajos niveles de oxígeno afectando el desarrollo de la planta (Villalobos et al., 2002). En este contenido de agua el potencial mátrico es cero y el gravitacional es el dominante (Plaster, 2013). Según Villalobos et al. (2002) and Delleur (2006), el $\theta_{\mathrm{S}}$ corresponde al 85\% de porosidad del suelo.

ii. Contenido de agua a capacidad de campo $\left(\theta_{\mathrm{CC}}\right)$, según su definición este es alcanzado dos o tres días después de un evento de lluvia o riego que llevo el suelo a saturación, es decir, cuando el drenado del agua se haya detenido, evitando las pérdidas de agua por evapotranspiración. (Duchaufour, 1998). En este nivel, el potencial mátrico y el gravitacional están en equilibrio, dando un potencial hídrico cercano a 1/3 bar. Este contenido de agua es el ideal para el crecimiento de la planta, ya que existe suficiente aire y el agua es más libre debido a su alto potencial (Plaster, 2013).

iii. Contenido de agua en el punto de marchitamiento permanente ( $\left.\theta_{\mathrm{PMP}}\right)$, es el contenido en el cual la planta muere de forma irreversible, debido a que la poca agua almacenada en el suelo está fuertemente ligada a las partículas del suelo y no puede ser absorbible por las raíces de la planta (Duchaufour, 1998) ver Fig. 4. El valor del potencial hídrico es usualmente de -15 bars, variando acorde a las plantas. El agua sigue agotándose hasta alcanzar el agua higroscópica donde es retenida entre los - 31 y -10,000 bars y sólo puede ser removida cuando el suelo es secado en horno (Plaster, 2013).

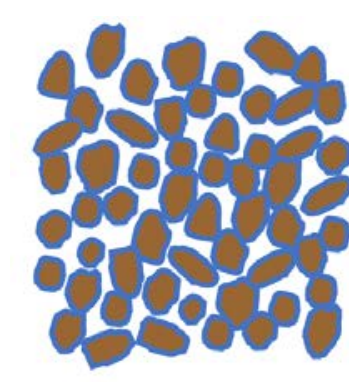

Punto de marchitamiento permanente

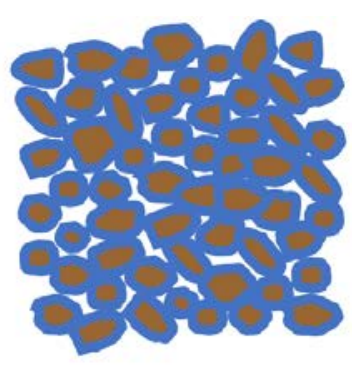

Capacidad de campo

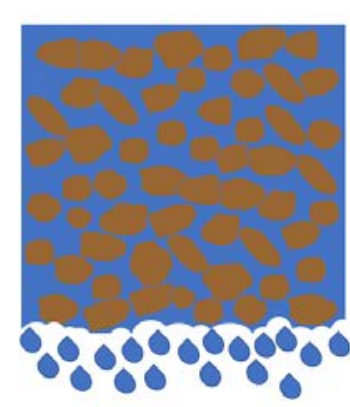

Saturación

Figura 4. Contenidos de humedad en el suelo. 
El contenido de humedad útil del suelo en la cual el cultivo pueda extraer el agua está entre $\theta_{\mathrm{CC}}$ y $\theta_{\mathrm{PMP}}$ y es denominado como humedad aprovechable del suelo (HA); en suelo arenosos, la HA es baja debido a su textura gruesa, mayor para los limos y arcillas (Duchaufour, 1998; Fuentes Ruíz et al., 1997). Sin embargo, no todos los cultivos son capaces de extraer todo ese contenido de agua resultante, es decir, algunos cultivos perecen en limites superiores al $\theta_{\text {PMP }}$ por lo que es necesario agregarle un factor que fraccione la HA del suelo, este factor indica cuando el cultivo comienza a mostrar síntomas de clorosis, afecciones en el crecimiento, marchitez, entre otros (Fuentes Ruíz et al., 1997).

En la Fig. 5 se muestra un resumen de los apartados anteriores que describen los estados del agua en el suelo.

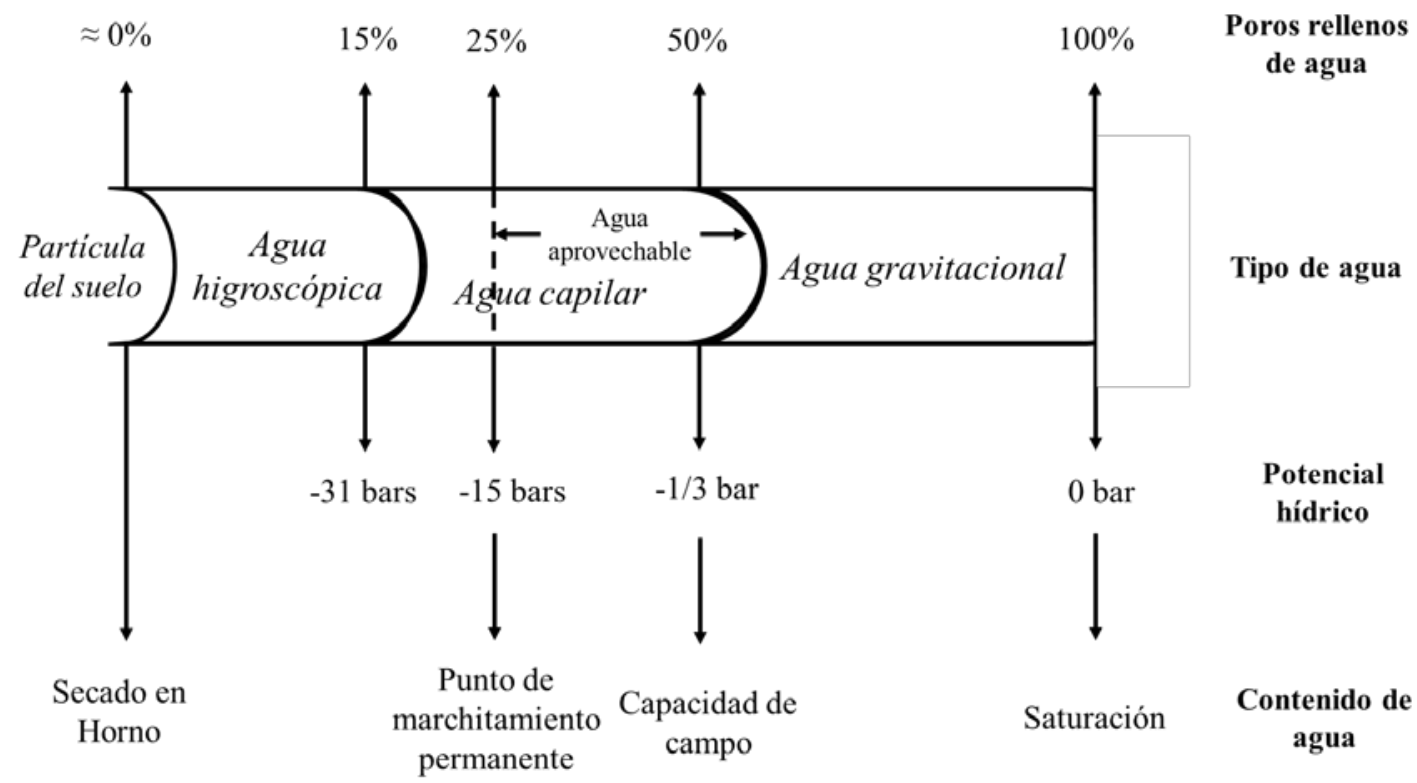

Figura 5. Contenido de agua, potencial hídrico y poros en función al tipo de agua en el suelo.

Moreno et al. (1978) establecieron una relación entre la retención de humedad y la textura del suelo en el margen del agua no disponible (agua higroscópica entre 4.2 y 6.0 pF). Ellos han encontrado una fuerte dependencia del contenido de humedad y las partículas finas. Cuando el suelo está entre 4.2 y 4.6 pF, las partículas menores a $0.02 \mathrm{~mm}$ son las que dominan la retención de humedad, y para tensiones que van de 4.6 a 6.0 pF las partículas que controlan la retención de humedad son las menores a $0.002 \mathrm{~mm}$. 


\subsubsection{Frente húmedo}

El agua que está ligada a la partícula sólida del suelo se va haciendo más grande con el paso del tiempo, por lo que un determinado momento suelta el agua y permite el avance de ésta, gracias al empuje de la gravedad y arrastre de la capilaridad que hace que el flujo de agua sea mueva en todas direcciones, definiendo a su paso un frente húmedo y dejando atrás un suelo casi saturado. Durante, el avance del agua a través del suelo va sufriendo pérdidas por fricción, en suelos con poros pequeños como los suelos arcillosos sufre más pérdidas, por lo que el avance del agua es lento, en cambio en suelos arenosos con poros más grandes el movimiento del frente húmedo es más rápido (Plaster, 2013), esto es reflejado en la Fig. $6 a$ y $6 b$.

En la Fig. 6c, el agua es fuertemente retenida en los pequeños poros de los suelos arcillosos por lo que al llegar al horizonte arenoso el frente húmedo tiende expandirse a los costados en lugar de ser arrastrado a los grandes poros de suelo arenoso, usualmente el agua se mueve de los grandes a los pequeños poros por el potencial matricial. Cuando el horizonte superior este saturado, el potencial gravitacional será lo suficientemente alto para arrastrar el agua por gravedad a la arena (Plaster, 2013).

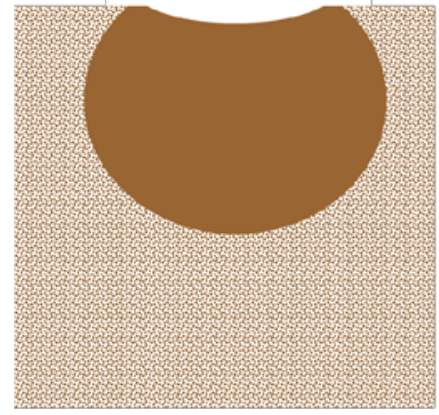

a) Suelo arcilloso

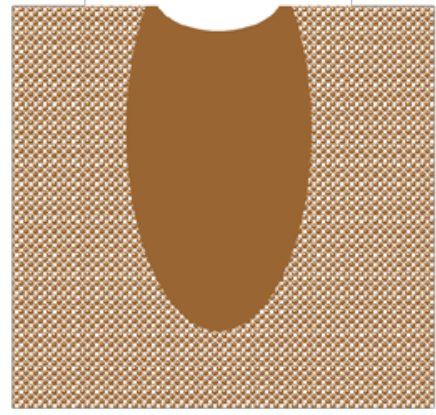

b) Suelo arenoso

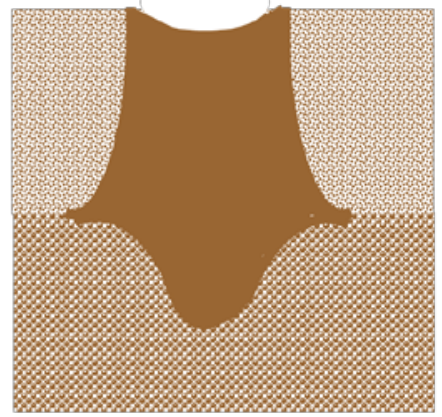

c) Suelo arcilloso y arenoso

Figura 6. Avance del frente húmedo en diferentes horizontes del suelo: a) textura fina, b) textura gruesa y c) horizonte inferior de textura fina e inferior de textura gruesa. 


\subsection{Infiltración}

Al proceso de entrada de agua en el suelo se le conoce como infiltración, así que la cantidad de agua infiltrada en un determinado tiempo se le denomina infiltración acumulada (I), y la infiltración por unidad de tiempo es dada por la velocidad de infiltración, (VI) (Kirkham, 2010; Smith et al., 2002), esta relación es descrita en la siguiente Ecuación 3:

$$
V I=\frac{d I}{d t}, \quad I=\int_{0}^{t} V I d t
$$

En las primeras etapas de la entrada del agua en un suelo relativamente seco domina el gradiente matricial sobre la gravitacional (Kirkham, 2010; Smith et al., 2002). A medida que el frente húmedo va avanzando el potencial mátrico va disminuyendo, ya que el potencial gravitacional va aumentado en la zona húmeda (casi saturada) del frente húmedo, por lo que la infiltración puede considerarse un movimiento vertical descendente (Ferguson, 1994; Smith et al., 2002). Conforme el tiempo pasa la infiltración se estabiliza a una velocidad básica o constante inducida por la gravedad que se aproxima a la conductividad hidráulica (Ferguson, 1994). La infiltración no ocurre en condiciones de saturación (Smith et al., 2002).

La infiltración del agua en el suelo es variable en espacio y tiempo, debido a la textura del suelo, conductividad hidráulica saturada, existencia de diferentes estratos o horizontes con diferente textura, prácticas culturales, porosidad, densidad aparente, variaciones del clima, grado de compactación, contenido inicial de agua en el suelo, materia orgánica, entre otras (Mattar et al., 2015; Tarjuelo Martín-Benito, 1991; Yuan et al., 2019).

La velocidad de infiltración del agua está condicionada al contenido de humedad presente en el instante que comience el ingreso de agua en el perfil del suelo, un suelo seco tendrá mayor velocidad de infiltración ya que el agua se mueve hacia a una parte donde el suelo tiene un potencial más bajo. La velocidad de infiltración (dI) básica es mayor para suelos de textura gruesa y baja en suelos de textura fina (Villalobos et al., 2002). La compactación disminuye la VI, ya que se obstruyen los grandes poros que sirven de canales 
por donde el agua se mueve. La materia orgánica mejora la estructura del suelo y por lo tanto la VI (Plaster, 2013).

La infiltración puede ser determinada indirectamente mediante la solución numérica de las ecuaciones del flujo en el medio poroso (Smith et al., 2002). El primer modelo en describir velocidad del agua en el suelo fue la ley Darcy obtenida experimentalmente en suelo saturado en 1856, usando una columna de suelo vertical de longitud L y de área transversal A, ver la Fig. 7. Esta ley establece que el flujo de agua entrante a un suelo saturado es linealmente proporcional a la sección por la cual se está moviendo y al gradiente hidráulico (dh/L), entendiéndose por este último como la pendiente entre los dos puntos en los cuales se está midiendo la altura del agua (Ecuación 4). Cada tipo de suelo tiene una constante de proporcional lineal especifico (K), Darcy lo denominó como conductividad hidráulica e indica la habilidad que tiene el suelo a transmitir el agua en un suelo saturado (Ferguson, 1994; Kirkham, 2010).

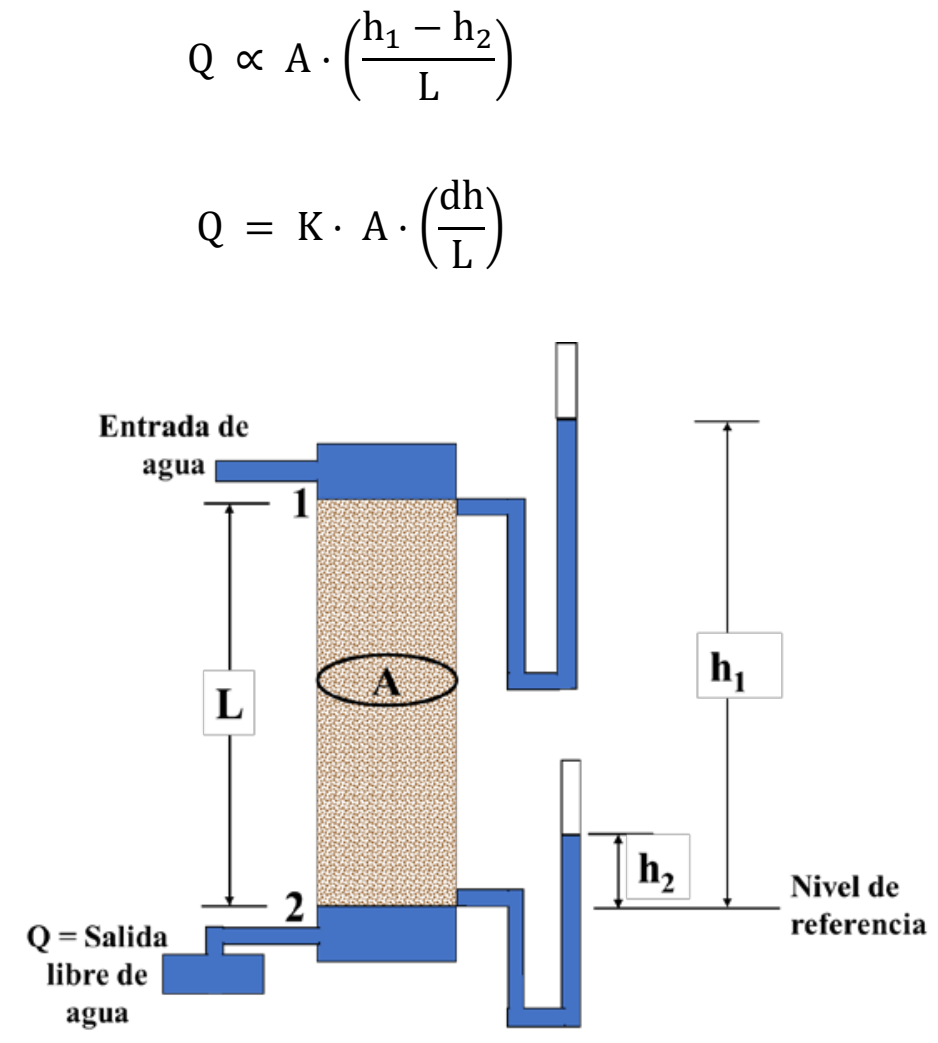

Figura 7. Experimentación para la ley de Darcy. 
En términos de velocidad del flujo por unidad de área, la ecuación de Darcy puede ser rescrita usando la equivalencia de la ecuación de continuidad (Ecuación 5), resultando la Ecuación 6 (Ferguson, 1994; Koorevaar et al., 1999; Smith et al., 2002):

$$
\begin{gathered}
\mathrm{Q}=\mathrm{v} \cdot \mathrm{A} \\
\mathrm{v}=\frac{\mathrm{Q}}{\mathrm{A}}=\frac{\mathrm{K} \cdot \mathrm{A} \cdot\left(\frac{\mathrm{dh}}{\mathrm{L}}\right)}{\mathrm{A}}=\mathrm{K} \cdot \frac{\mathrm{dh}}{\mathrm{L}}
\end{gathered}
$$

donde v es la velocidad del agua, $\mathrm{Q}$ es el caudal de agua ingresado, $\mathrm{h}$ representa el potencial de energía comprendido entre el potencial mátrico más el potencial gravitacional y L es medido en dirección del flujo vertical con z positivo hacia abajo, tenemos que (AnguloJaramillo et al., 2016; Smith et al., 2002):

$$
\mathrm{h}=\Psi-\mathrm{z}
$$

La conductividad hidráulica debe estar en función del contenido de humedad, para ser usada en flujo no saturado (Delleur, 2006; Tarjuelo Martín-Benito, 1991), la ecuación resultante es denominada la ecuación de Darcy-Buckingham quedando de la siguiente manera:

$$
\mathrm{v}=-\mathrm{K}(\theta)\left(\frac{d \Psi}{d z}-1\right)
$$

Cuando un suelo no está saturado los valores del $\Psi$ son menores que cero, esto porque en un suelo no saturado domina más el potencial matricial que el gravitacional (Delleur, 2006; Tarjuelo Martín-Benito, 1991).

Años después Richards (1931) estableció su modelo del movimiento de agua en suelo no saturados (Ecuación 10), conjugando la ley de Darcy-Buckingham (Ecuación 8) y la ecuación de continuidad en dirección vertical (Ecuación 9). Se establece un flujo de agua vertical descendente con coordenadas conocidas que parten de la superficie del suelo. En este modelo son implicados los contenidos de humedad $(\theta)$, el cabezal de succión y la 
conductividad hidráulica del suelo (K). Asumiendo un suelo homogéneo, uniforme, material poroso inerte y con un efecto del movimiento en el aire insignificante (Ali, 2010; AnguloJaramillo et al., 2016; Smith et al., 2002).

$$
\begin{gathered}
\frac{\partial \theta}{\partial \mathrm{t}}=-\frac{\partial \mathrm{q}}{\partial \mathrm{z}} \\
\frac{\partial \theta}{\partial \mathrm{t}}=\frac{\partial}{\partial \mathrm{z}}\left(\mathrm{K}(\theta)\left(\frac{\partial \Psi}{\partial \mathrm{z}}-1\right)\right)
\end{gathered}
$$

Algunos de los parámetros del modelo de Richards requieren de experimentación detallada, por lo que modelos simplificados basados en propiedades físicas del suelo, fueron propuesto para describir la infiltración del agua unidireccional del suelo; entre los cuales destacan Kostiakov, Kostiakov Modificado, Lewis (1937), Philip (1957), Horton (1941), Mahmood and Latif (2005), Mattar et al. (2015), entre otros.

El modelo de Kostiakov fue desarrollado a partir de las observaciones del volumen infiltrado y el tiempo, por lo que no tiene un significado físico con respecto al suelo (Kirkham, 2010). A partir de los datos obtenidos en la experimentación fue observado que tendían a un valor final constante, así que al modelo original de Kostiakov le fue agregado una constante al final y se le denomino Kostiakov Modificado. Horton (1941) estableció su modelo de infiltración para nivel de cuenca, este modelo es resultado de simplificaciones realizadas a la ecuación de continuidad. En el modelo de Philip es asumido una condición inicial de encharcamiento (Kirkham, 2010).

Existen diferentes trabajos de investigación donde hacen mejoras a las ecuaciones típicas de determinación de infiltración de agua en el suelo. Yang, et al. (2020) establece una relación ente la capacidad de infiltración y el contenido de humedad para mejorar la ecuación de Horton. También existen trabajos donde se evalúan estos modelos típicos de infiltración como el trabajo de Babaei et al. (2018), el cual fue enfocado a encontrar el mejor modelo representativo para las tierras áridas de Irán y al mismo tiempo analizar la variabilidad espacial del proceso de infiltración. En este trabajo utilizan los modelos de Philip, Horton, Kostiakov, Kostiakov Modificado y Kostiakov Modificado Revisado mediante el uso de un 
infiltrómetro de tensión. El mejor modelo de predicción fue el de Kostiakov Modificado Revisado y además de ser el mejor para determinar la infiltración acumulada.

El efecto que tiene la vegetación en la superficie o el tipo de estructura del suelo sobre la velocidad de infiltración del agua de fue evaluado por de Almeida et al. (2018). En este trabajo fue analizada la VI de una intensidad de lluvia constante en un suelo desnudo, otro cultivado con labranza, otro sin labrar, y uno último con pasto. La infiltración acumulada fue determinada como la diferencia ente la lluvia artificial y el escurrimiento superficial. Este último parámetro fue calculado a partir de relación entre el volumen recolectado y el área experimental. Los datos de infiltración observados fueron ajustados a las ecuaciones de Kostiakov Modificada, Horton y Philip. La mayor velocidad de infiltración estable o básica encontrada fue cuando el suelo estaba sembrado sin labranza, en cambio las velocidades de un suelo cultivado con labranza y un suelo desnudo no difieren mucho. El modelo que mejor se ajusta es el de Horton. Concluyen que la cubierta vegetal es la que más influye en la velocidad de infiltración independientemente del suelo y no tanto por el tipo de labranza.

De manera parecida, Li et al. (2018) determinaron la infiltración en suelos francolimosos, arenosos y una mezcla de ambos, en algunos de estos suelo les fue agregado una capa de octadecilamina para simular una capa hidrofóbica (repelente al agua). Los perfiles de suelo fueron construidos dentro de una botella de Marriot ya que entrega una tasa contante de agua. Así que la infiltración acumulada fue determinada a partir de la diminución del volumen de agua en un tiempo específico dividido por el área de la sección transversal de la botella. El avance del frente húmedo fue determinado visualmente con respecto al tiempo. El frente húmedo alcanzó los $45 \mathrm{~cm}$ en suelos sin capa repelente y $35 \mathrm{~cm}$ en aquellos suelos que si tenían dicha capa. Los perfiles de suelo con textura franco-limosa y arenosa produjeron zonas de transición bruscas del contenido de agua. La infiltración acumulada y la VI en un suelo franco limoso con capa repelente al agua disminuyeron conforma aumentaba el tiempo, concluyen que la posición de las capas del suelo tiene menor importancia con respecto a la capa de repelencia al agua en el efecto al comportamiento de la infiltración.

Cui et al. (2019) encontraron que la velocidad de infiltración de agua en el suelo (VI) es afectada por el contenido de humedad del suelo y principalmente por el volumen de raíces. 
Las raíces con diámetro mayores a 4.5 mm tuvieron una correlación negativa con la VI, en cambio la VI inicial era afectada por el contenido de agua en el suelo, disminuyendo conforme el tiempo de infiltración va aumentando. Las VI fueron determinadas de forma automática a través de un sistema que consistía en una computadora, una cámara, un trípode y una bomba peristáltica, en cinco diferentes pastizales artificiales. Una bomba suministraba agua al suelo a una tasa constante, y la cámara controlada por la computadora capturaba imágenes cada 3 minutos. El algoritmo de determinación de VI estaba basado en el cambio de humedad del área, el gasto entrante y el tiempo. Todo esto fue realizado en una región de la meseta de China y en un suelo franco areno-limoso con una temperatura anual promedio de $9{ }^{\circ} \mathrm{C}$ y precipitaciones anuales de $584 \mathrm{~mm}$

Al igual que en el trabajo anterior, Liu et al. (2019) encontraron la relación entre el contenido de humedad del suelo y la raíces de tres diferentes pastizales en un suelo arenoso y bajo condiciones frías de la Región Autónoma de Mongolia en China. El contenido de humedad es el principal factor que influye en la velocidad de infiltración inicial y para la velocidad estable influyen la humedad y las raíces. La influencia de ambos disminuye con la duración del tiempo de infiltración. La velocidad de infiltración fue determinada con el mismo sistema que utilizo Cui et al. (2019) y el mismo algoritmo.

Una simulación de la infiltración en un suelo heterogéneo con contenido inicial arbitrario, lluvia inestable y de drenaje libre fue realizada por Herrada et al. (2014). Utilizaron el método unidimensional de Richards para el cálculo de la VI. En condiciones de drenaje libre, asumen una variación del contenido de agua con respecto a la profundidad es igual a cero y la profundidad es igual a L en el fondo. Por lo tanto, la velocidad de infiltración es igual a la intensidad de la lluvia en el límite superior donde z es igual a cero y las condiciones de humedad menores a la saturación. Cuando el suelo está saturado la infiltración es determinada como lluvia menos escurrimiento superficial. La humedad del suelo en cualquier profundidad es determinada a partir del cambio de los flujos que atraviesa los limites superior e inferior del perfil del suelo. Los flujos son determinados a partir de las mediciones de la conductividad hidráulica del suelo y del cabezal de presión en saturación. 
Un sistema para simular los procesos de infiltración, ascenso capilar, evapotranspiración y escurrimiento superficial fue desarrollado por del Valle Paniagua, et al. (2009) y sus resultados fueron validados con valores reales de arcilla y arena. La infiltración fue modelada con la ecuación de Richards, el ascenso capilar con la ley de Darcy ya que el agua asciende desde el manto freático y se comporta como un suelo saturado, para la modelación de la evapotranspiración potencial se utilizó la ecuación de Penman y de manera independiente con datos de precipitaciones se modelo el escurrimiento superficial. Su sistema desarrollado responde preguntas como: ¿cuál es la cantidad de escurrimiento superficial? y ¿cuándo se produce?, ¿para qué tipos de superficie se producen estancamientos?, ¿de qué magnitud son? y ¿cuándo suceden?, ¿cómo se comportan las capas del suelo? y ¿qué sucede cuando una de ellas se satura? y ¿cómo cambia la distribución del agua en el suelo a través del tiempo?

Entre los dispositivos más usados para obtener información de la infiltración in situ por su fácil manejo son los infiltrómetros, y estos pueden ser de uno o dos anillos (Fig. 8). Los infiltrómetros trabajan en condiciones de encharcamiento, es decir, los anillos o cilindros de metal son hincados dentro del suelo a poca profundidad (hasta $10 \mathrm{~cm}$ ), después son rellenados de agua y la velocidad con que el agua se va moviendo dentro del suelo es registrada (Ali, 2010; Bagarello and Sgroi, 2004), a partir de la Ecuación 11:

$$
\text { VI }=\left(\text { Lámina Infiltrada }_{\mathrm{i}-1}-\text { Lámina Infiltrada }_{\mathrm{i}}\right) /\left(\text { Tiempo }_{\mathrm{i}-1}-\text { Tiempo }_{\mathrm{i}}\right)
$$

donde la lámina infiltrada representa el volumen de agua que ha entrado en un tiempo determinado.

Al paso del tiempo en estas pruebas in situ, la velocidad de infiltración se establece en una velocidad estable denominada conductividad hidráulica (Seiler and Gat, 2007). El flujo del agua por debajo del anillo no es únicamente vertical, esto por no haber paredes que obliguen al movimiento vertical, así que las fuerzas de capilaridad del suelo circundante hacen que el agua se mueva de manera lateral. En el caso del infiltrómetro de doble anillo trata de eliminar el error antes mencionado, al colocar un anillo externo con agua entre los 
dos anillos que forzará el movimiento vertical del agua que está dentro del anillo central (Ali, 2010).

Sin embargo, durante el hincado de los anillos de metal en el suelo se crea una compactación en suelos húmedos y fracturación de la macroestructura en suelos secos (Bagarello and Sgroi, 2004).

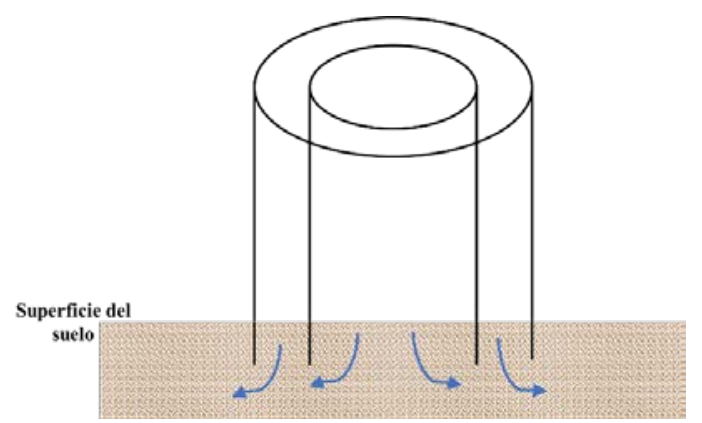

Figura 8. Infiltrómetro de doble anillo.

En el trabajo de Arriaga et al. (2010) se determinó la VI y conductividad hidráulica saturada de dos diferentes suelos, franco-arenoso y arcilloso, por medio de un infiltrómetro de doble anillo con condiciones de caída de cabezal, es decir, el nivel del agua medido dentro del anillo central disminuye en función del tiempo. Atribuyen las diferencias de los resultados en las propiedades hidráulicas, a la densidad aparente del suelo y al tamaño y distribución de las partículas. Los suelos con mayor contenido de arena y baja densidad aparente tienen mayor velocidad de infiltración casi-estable, infiltración acumulada y conductividad hidráulica a saturación. La conductividad a saturación fue determinada a partir de una ecuación que toma valores del radio del cilindro, profundidad de inserción del anillo, longitud de capilaridad macroscópica del suelo que es de $0.12 \cdot \mathrm{cm}^{-1}$ para la mayoría de los suelos agrícolas y dos constantes empíricas adimensionales que depende de la profundidad de inserción y de encharcamiento. Otros trabajos acerca de la determinación de la infiltración usando diferentes infiltrómetros son los de Mahmood and Latif (2005) y Fatehnia et al. (2016).

En al algunas investigaciones utilizan el lisímetro para medir el flujo de drenado del agua del perfil de un suelo no saturado, como el trabajo de Masarik et al. (2004). A través de un lisímetro de equilibrio de tensión ajustan continuamente la tensión del lisímetro al 
potencial mátrico del suelo circundante mediante un sensor disipador de calor que miden el potencial mátrico y una bomba que aplica succión al lisímetro. La succión comenzaba a los $2 \mathrm{kPa}$ y paraba a los $-35 \mathrm{kPa}$. El ajuste automático de la tensión del lisímetro permite mantener un equilibrio casi constante entre el suelo dentro del lisímetro y el suelo circundante, lo que proporciona una recogida más precisa del drenaje sin ser subestimada.

\subsection{Evapotranspiración del cultivo}

El método más eficaz en la programación del riego es basarlo en el consumo hídrico del cultivo, es decir, en la evapotranspiración (ET). El objetivo es compensar con riego las pérdidas de agua por ET del cultivo, que engloba dos procesos: i) pérdidas de agua por evaporación del suelo donde está creciendo el cultivo y ii) pérdidas de agua por la transpiración del cultivo, ambos procesos ocurren simultáneamente y es difícil distinguir entre uno y otro. Al hacer esto, se trata de mantener las mismas condiciones humedad en el suelo antes de sufrir dichas pérdidas de agua (Allen et al., 1998; Shukla et al., 2013), claro está que dichas condiciones de humedad deben estar dentro de la humedad aprovechable para el cultivo.

A medida que aumenta el área foliar del cultivo la evaporación del suelo va disminuyendo, esto se debe a que es principalmente determinada por la radiación solar que impacta sobre el suelo; en cambio la transpiración aumenta a medida que el cultivo se va desarrollando (Allen et al., 1998)

La evapotranspiración puede clasificarse en función a las condiciones en la que es determinada:

i. Evapotranspiración de referencia $\left(\mathrm{ET}_{\mathrm{O}}\right)$, refiere a la evapotranspiración de un cultivo hipotético similar a un pasto verde de gran extensión, abordando la demanda evaporativa del clima independientemente del tipo de cultivo. El cultivo hipotético asume una altura uniforme, bien regado, creciendo activamente y 
uniformemente y causando sombra sobre toda la superficie del suelo (Pereira et al., 2010).

El método estándar para determinar la $\mathrm{ET}_{\mathrm{O}}$ es el de FAO Penman-Monteith, éste utiliza datos de temperatura del aire, humedad relativa del aire, velocidad del viento, horas luz y radiación solar. La $\mathrm{ET}_{\mathrm{O}}$ también puede ser determinada a partir de datos de un tanque evaporímetro, condicionado a un buen manejo del tanque (Allen et al., 1998).

ii. Evapotranspiración del cultivo $\left(\mathrm{ET}_{\mathrm{C}}\right)$, es la ET referida a cualquier cultivo que este bajo buenas condiciones, es decir, buena fertilización, óptimas condiciones de humedad en el suelo, sin enfermedades, creciendo en grandes extensiones de tierra y que alcanza su máximo rendimiento, tomando en cuenta las afectaciones por condiciones climáticas. $\mathrm{La}_{\mathrm{ET}} \mathrm{C}$ es la relación entre la $\mathrm{ET}_{\mathrm{O}} \mathrm{y}$ un coeficiente de cultivo $\left(\mathrm{K}_{\mathrm{C}}\right)$, éste último caracteriza el crecimiento del cultivo en sus diferentes etapas de fenológicas y resume las diferencias fisiológicas entre cultivos (Allen et al., 1998).

iii. Evapotranspiración real o actual, $\left(\mathrm{ET}_{\mathrm{a}}\right)$, es la ET del cultivo producida bajo condiciones diferentes a las antes mencionadas. El valor de $\mathrm{ET}_{\mathrm{a}}$ se determina de la misma forma que la $\mathrm{ET}_{\mathrm{C}}$, sin embargo, hay que afectarlo por un coeficiente de estrés hídrico $\mathrm{K}_{\mathrm{S}}$ o hacer un ajuste al $\mathrm{K}_{\mathrm{C}}$ si presenta condiciones adversas a las óptimas, las cuales pueden ser la presencia de salinidad del suelo, plagas o enfermedades, estrés hídrico o exceso de agua, entre otras (Allen et al., 1998).

La programación del riego a partir de la $\mathrm{ET}_{\mathrm{C}}$ es buena si existe una medición precisa de este parámetro. A partir de las variaciones másicas de un lisímetro de pesada de precisión, y con un balance hídrico es posible determinar la evapotranspiración con muy buena exactitud (Allen et al., 1998). Este método es ampliamente utilizado alrededor del mundo como parámetro determinante en el requerimiento hídrico del cultivo. A continuación, se verán algunos trabajos referentes al cálculo de la $\mathrm{ET}_{\mathrm{C}}$ para distintos cultivos y diferentes condiciones climatológicas, esto para entender la importancia que tienen esas variables en la 
determinación de $\mathrm{ET}_{\mathrm{C}}$. Además de otros trabajos donde son determinadas diferentes variables consideradas en el balance hídrico del suelo.

En los trabajos de Herbrich and Gerke (2016), Hannes et al. (2015), Peters et al. (2014) y Schrader et al. (2013) determinaron la evapotranspiración del cultivo y la lluvia a partir de los datos de masa de los dos recipientes de un lisímetro de pesada. El flujo de agua dentro de los recipientes es determinado como la diferencia de la masa en dos puntos de tiempo. Cuando la suma de la variación de masa de los dos recipientes con respecto al tiempo es mayor a cero indica que ha ingresado agua de lluvia, en cambio, cuando la suma es menor a cero indica que está ocurriendo pérdidas de agua por parte de la evapotranspiración del cultivo.

La lluvia es un parámetro presente en el balance hídrico del cultivo, en el trabajo de Hoffman et al. (2016) determinan la lluvia como se indica en el trabajo de Peters et al. (2014) en tres lisímetros que tiene área de $1 \mathrm{~m}^{2}$ y la comparan con la registrada en tres pluviómetros Hellman simples con área de captación de $100 \mathrm{~cm}^{2}$ y una altura de $33 \mathrm{~cm}$, y con un pluviómetro de cubo basculante de $200 \mathrm{~cm}^{2}$. Encontrando que los datos de precipitación obtenidos con la metodología de Peters et al. proveen una alta exactitud posible si son evitados errores sistemáticos (distorsiones por el viento o pérdida por evaporación). Los pluviómetros Hellman que fueron colocados directamente en la superficie del suelo tiene una comparable captación y precisión de medición de la lluvia como la del lisímetro por lo que podría ser una opción para grandes periodos. Sin embargo, Ducho and Biddle (2010) determinaron que los pluviómetros de cubo basculante llegan a ser insuficientes cuando la lluvia excede una intensidad de $50 \mathrm{~mm} \cdot \mathrm{h}^{-1}$ y cuando el viento supera una velocidad de $5 \mathrm{~m} \cdot \mathrm{s}^{-1}$. De manera similar en el 2007, Meissner et al. midieron la precipitación que se produce en forma de rocío, niebla y escarcha a través de un lisímetro. Estos eran determinados por los pequeños incrementos de masa y cuando las condiciones climáticas eran propicias.

En cuanto a la escorrentía superficial, en el trabajo de Adekalu, et al. (2007) analizaron el efecto de acolchonado producido por la hierba en la infiltración, la escorrentía superficial y erosión del suelo. Probaron tres tipos de suelo con pendiente, con la intensidad 
de lluvia típica de la zona y con diferentes volúmenes de hierba. El volumen de agua infiltrada se calculó como la diferencia entre el volumen de agua añadida y la suma de la escorrentía y el volumen interceptado por el mantillo de residuos de hierba. Existió una alta correlación entre las tres variables, la escorrentía y la erosión del suelo disminuyeron con la cantidad de hierba, pero aumentaron con la pendiente. La escorrentía tenía una correlación negativa con el contenido de arena, pero positivamente con el contenido coloidal (materia orgánica y arcilla). La infiltración aumentó y la erosión se redujo grandemente mientras mayor era la cobertura se hierba. Los residuos de hierba de elefante aumentan el almacenamiento de agua en el suelo y ayudar a reducir la erosión.

Entre las metodologías indirectas en la determinación de la $\mathrm{ET}_{\mathrm{C}}$ está el método estándar propuesto por Allen et al. (1998), en el Manual 56 de la FAO (Food and Agriculture Organization of the United Nations). El método consta de dos pasos, primero es determinado la evapotranspiración de cultivo de referencia $\left(\mathrm{ET}_{\mathrm{O}}\right)$, en el cual se asume un cultivo hipotético similar al pasto que creciendo en buenas condiciones de humedad y con una altura uniforme. $\mathrm{La}_{\mathrm{ET}_{\mathrm{O}}}$ es calculada a partir de datos climáticos, como la humedad relativa del aire, horas luz, radiación solar, velocidad del viento y temperatura del aire; este método es llamado FAO Penman-Monteith y representa el efecto del clima en el requerimiento hídrico del cultivo. El segundo paso refiere a un coeficiente de cultivo $\left(\mathrm{K}_{\mathrm{C}}\right)$, que representa el efecto del cultivo sobre la $\mathrm{ET}_{\mathrm{C}}$ que integra propiedades físicas y fisiológicas del cultivo. Este parámetro puede ser determinado a partir de dos enfoques, el primero de ellos es denominado coeficiente único y es el cociente entre $\mathrm{ET}_{\mathrm{C}}$ y la $\mathrm{ET}_{\mathrm{O}}$; en el segundo enfoque, el $\mathrm{K}_{\mathrm{C}}$ es la suma del efecto de la evaporación $\left(\mathrm{K}_{\mathrm{e}}\right)$ y transpiración $\left(\mathrm{K}_{\mathrm{cb}}\right)$ sobre el cultivo.

Existen diferentes maneras de determinar la $\mathrm{K}_{\mathrm{C}}$, una de ellas es considera el estrés de la cosecha a través de las unidades térmicas fraccionales (FTU) o días grados de crecimiento de la temperatura media del aire. Las FTU miden el calor acumulado por encima de la temperatura de mínima (conocida como basal) que varía entre las especies de cultivos; el crecimiento de las plantas es nulo cuando la temperatura está por debajo de la temperatura basal. En el trabajo de Martínez-Cob (2008) se desarrollaron dos ecuaciones de segundo y tercer orden polinomial de $\mathrm{K}_{\mathrm{C}}$ diarios para el maíz en función de las FTU a partir de ET $_{\mathrm{C}} \mathrm{y}$ $\mathrm{ET}_{\mathrm{O}}$ medidos con el lisímetro. Ellos encontraron que los valores de $\mathrm{K}_{\mathrm{C}}$ determinados a partir 
de la metodología FAO ( $\mathrm{ET}_{\mathrm{C}}$ del lisímetro sobre la $\mathrm{ET}_{\mathrm{O}}$ de Penman-Monteith) son ligeramente peores a los que ellos validaron. Recomiendan evitarla para la programación del riego en tiempo real, esto porque con antelación la temporada fenológica del maíz debe ser divida en cuatro etapas, por lo que no se tendrían en cuenta las posibles variaciones que causarían las condiciones climáticas sobre el cultivo. Con el uso de las FTU se mejoraría la incertidumbre causada con la metodología de la FAO.

En este sentido, en la investigación de Mateos et al. (2013) fue determinada la evapotranspiración de diferentes campos con hortalizas (ajo y algodón) y huertas de árboles (melocotón, olivos y mandarina) en el sureste de España a partir de los índices vegetativos (IV) y la $\mathrm{ET}_{\mathrm{O}}$. Usando imágenes satelitales determinan la fracción de cobertura vegetal para estimar el $\mathrm{K}_{\mathrm{cb}}$ y el $\mathrm{K}_{\mathrm{e}}$ a partir de la metodología propuesta en el Manual 56 da la FAO. Los modelos obtenidos fueron validados con la ET medida con un sistema de Eddy covarianza que consiste de varios dispositivos que miden la velocidad del viento, la densidad de vapor de agua y temperatura del aire. El método $\mathrm{IV}-\mathrm{ET}_{\mathrm{O}}$ es bueno para determinar la variabilidad de ET para grandes zonas de riego. La parte débil del método es la determinación de la componente de la evaporación del suelo, ya que únicamente el enfoque del coeficiente doble puede ser aplicado cuando se conoce la fecha de humectación del suelo. Otro trabajo que utiliza las imágenes satelitales para determinar los índices vegetativos del cultivo y así calcular la evapotranspiración de los cultivos fue realizado por Kullberg et al. (2017)

En el trabajo de Fernández-Pacheco et al. (2014) utilizan el procesamiento de imágenes digitales para estimar los $\mathrm{K}_{\mathrm{C}}$ de la lechuga en una provincia de Murcia, España. Tomaban fotografías de la cobertura vegetal de una superficie de $1 \mathrm{~m}^{2}$, las imágenes eran procesadas para separar el suelo de la vegetación, usando el número de pixeles correspondientes a la vegetación $(\mathrm{V})$, al suelo y la suma de ambos $(\mathrm{T})$ era calculado el porcentaje de la cobertura del suelo, PGC. El $\mathrm{K}_{\mathrm{C}}$ fue determinado con la metodología de la $\mathrm{FAO}$, así que la $\mathrm{ET}_{\mathrm{C}}$ fue medida con una estación de razón de Bowen que mide el flujo de calor latente necesario para causar la evaporación del agua en el suelo calculado mediante el método de la Razón de Bowen-Balance de Energía y la $\mathrm{ET}_{\mathrm{O}}$ fue determinada con el método FAO Penman-Monteith a partir de los datos de una estación climatológica, ambas estaciones instaladas en el lugar. 
Una función de doble sigmoide fue obtenida para relación de PGC y la altura de la planta (h) ya que mostraban una distribución similar. De igual manera, el $\mathrm{K}_{\mathrm{C}} \mathrm{y}$ la relación de PGC/h mostraron una similar distribución, así que fue obtenida una función logarítmica de ambos. Esta metodología propuesta se ajusta a las condiciones climáticas del lugar y a las etapas de desarrollo de los cultivos. Esta investigación sirvió de base para el trabajo de Escarabajal-Henarejos et al. (2015), donde fue estimado el requerimiento hídrico de la lechuga a partir de los componentes del balance hídrico. La humedad aprovechable del suelo fue establecida con una fracción de agotamiento permisible de 0.15 de la capacidad de campo del suelo. Por lo tanto, los riegos fueron programados para para mantener en umbrales próximos la capacidad de campo, evitando llegar a niveles por debajo del máximo agotamiento permisible.

El método propuesto por Fernández-Pacheco et al. (2014) mostró ser una técnica sencilla que permitió la determinación uno de los parámetros del balance hídrico y así tener una óptima gestión de la lechuga como lo demostraron Escarabajal-Henarejos et al. (2015), obteniendo mayor productividad del cultivo y menor consumo de agua 



\section{Materiales y Métodos}

Las distintas regiones ubicadas en del Mediterráneo Español son caracterizadas por su creciente escasez de agua, sobreexplotación del recurso hídrico, y contaminación de los acuíferos a causa de los nitratos y salinización. Estas regiones exigen una apropiada gestión del riego para obtener producciones óptimas y económicas rentables, evitando daños al medio ambiente con aplicaciones excesivas de riego (Gallardo et al., 2013). A continuación, se mostrados los dos trabajos realizados para el desarrollo de esta Tesis y ayudar en la mejora del uso hídrico en la agricultura para estas regiones.

\subsection{Determinación de la infiltración del agua del suelo}

\subsubsection{Zona de estudio}

La zona de estudio fue la finca Las Tiesas cuya extensión es de 1 ha, localizada en Albacete, España (39 3' 31" N, 2 6' 04" O y con una elevación de 695 msnm) durante el año 2017 (Fig. 9). Albacete es caracterizado con un clima semiárido, con una lluvia anual 
promedio de $384 \mathrm{~mm}$, temperaturas altas en verano de hasta de $40{ }^{\circ} \mathrm{C}$, mínimas de $-6{ }^{\circ} \mathrm{C}$ y con una temperatura promedio de $30^{\circ} \mathrm{C}$.

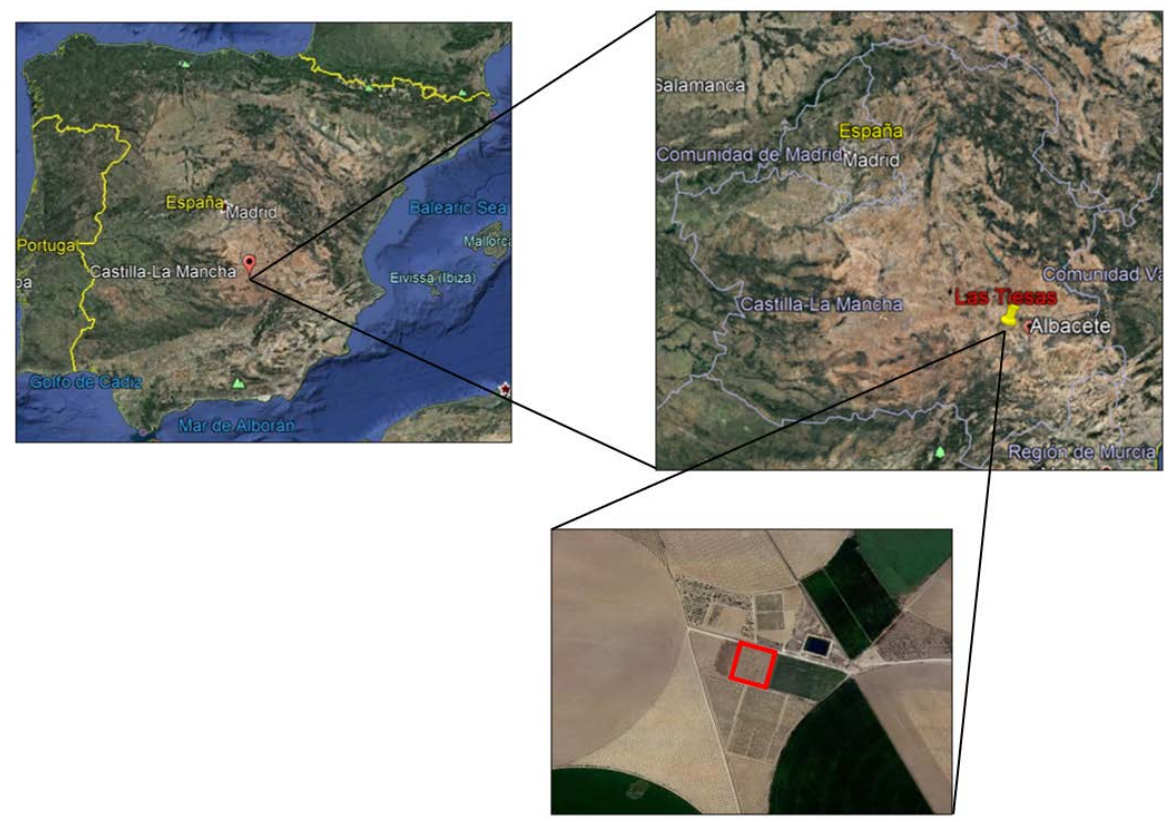

Figura 9. Zona de estudio, finca Las Tiesas en Alicante España.

El suelo fue clasificado con textura franco limosa a partir de los porcentajes del suelo obtenidos con un tamizado, el método del hidrómetro de Bouyoucos (27.1\% de arena, 51.5\% de limo y 21.4\% de arcilla) y el triángulo de textura de la USDA (Departamento de Agricultura de E.U.A.) (Staff, 1951); el contenido de materia orgánica fue de 2.77\% y una densidad aparente de $1.38 \mathrm{~g} \cdot \mathrm{cm}^{-3}$. Los contenidos de agua a capacidad de campo y punto de marchitez permanente fueron obtenidos por los métodos de la membrana y olla a presión resultando en $0.35 \mathrm{~m}^{3} \cdot \mathrm{m}^{3}$ y $0.16 \mathrm{~m}^{3 \cdot} \mathrm{m}^{-3}$, respectivamente. El contenido de humedad inicial del suelo, del día 8 de febrero cuando inicia el experimento, fue inferido de la densidad aparente del suelo y del volumen total que ocupa el suelo en el lisímetro, quedando en $0.23 \mathrm{~m}^{3} \cdot \mathrm{m}^{-3}$. 


\subsubsection{Materiales}

El lisímetro de pesada compacto usado en esta investigación, modelo LisITN-AC-E 1 (Telenatura, Alicante, España) consta de dos recipientes. El recipiente superior es denominado recipiente de suelo y tiene dimensiones de $0.56 \mathrm{~m} \times 0.96 \mathrm{~m}$ y una profundidad de $0.30 \mathrm{~m}$ en los extremos y $0.35 \mathrm{~m}$ en el centro, el cual almacena un volumen de suelo reconstituido de la parcela experimental intentado mantener las mismas condiciones de donde fue extraído (Fig. 10). El suelo dentro del recipiente está aislado de su ambiente de tal manera que los flujos laterales y flujos del ascenso capilar son cero (Ruiz-Peñalver et al., 2015).

El segundo recipiente, denominado depósito de drenaje, está localizado por debajo del recipiente del suelo, el cual sirve para almacenar el exceso de agua drenado por el perfil del suelo. El recipiente del suelo tiene un agujero en la parte central del fondo que permite el flujo del agua hacia a una electroválvula, después a un embudo y finalmente llega al depósito de drenaje (ver Fig. 10c). El sistema de medición del depósito de drenaje fue configurado para que cuando un almacenamiento de $3000 \mathrm{~g}$ de agua fuera reportado, la electroválvula localizada debajo de este depósito se abriera y comenzara el vaciado del agua; mientras esto ocurre la electroválvula del recipiente del suelo es cerrada para para evitar pérdidas de cuantificación de agua.

Las variaciones de masa de ambos recipientes son detectadas por células de carga, cuatro para el recipiente del suelo y una para depósito de drenaje. Las células de carga envían una señal eléctrica al datalogger (modelo CR300, Campbell Scientific, Logan, UT, USA) acorde a la deformación causada por el objeto que ellas soportan, esta señal eléctrica es convertida a un valor de masa por el propio datalogger. La resolución de medición de recipiente del suelo es de 20 g (0.033 mm) y para el depósito de drenaje es de 1 g (0.002 mm). Basados en la norma española UNE 500520 (AENOR, 2002) los datos de masa de ambos recipientes fueron muestreados cada segundo y el promedio de 60 segundos (un minuto) era registrado como un valor instantáneo. Esto garantiza la máxima calidad y representatividad de los datos obtenidos. Para más detalles constructivos del este lisímetro de pesada compacto revisar el trabajo de Nicolás-Cuevas et al., (2020). 

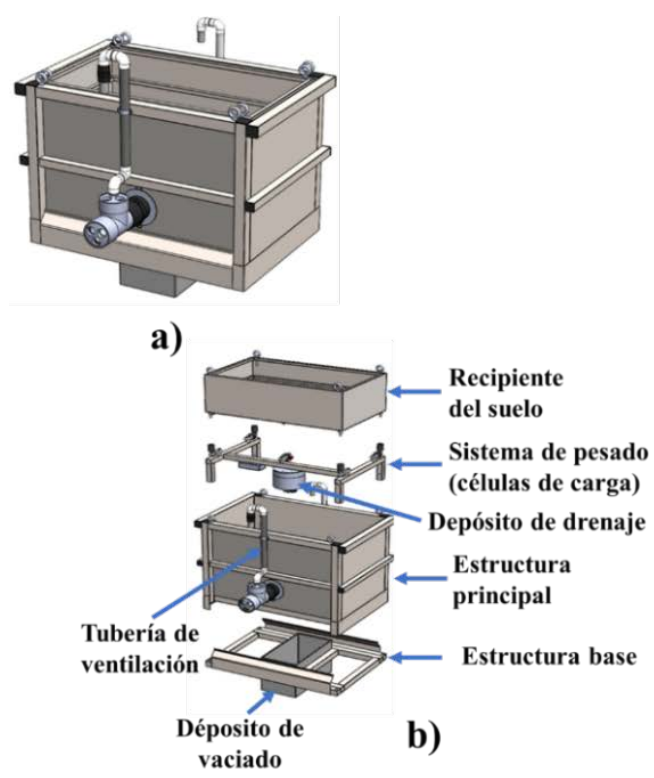

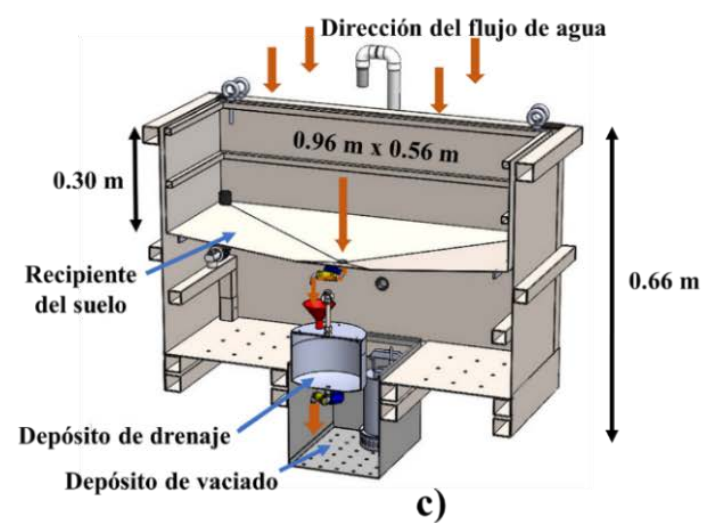

c)

Figura 10. Lisímetro de pesada compacto, a) vista general, b) vista detallada y c) movimiento del agua a través de los recipientes.

\subsubsection{Cálculo de los flujos de agua}

El lisímetro contiene un suelo aislado, por lo que es posible determinar las entradas y salidas de agua del suelo basados en los registros de los datos de masa, mediante la ecuación de balance de masa, con la Ecuación 12:

$$
\frac{\mathrm{dS}}{\mathrm{dt}}=\frac{\mathrm{dP}}{\mathrm{dt}}+\frac{\mathrm{dIR}}{\mathrm{dt}}-\frac{\mathrm{dD}}{\mathrm{dt}}-\frac{\mathrm{dET}}{\mathrm{dt}}-\frac{\mathrm{dSr}}{\mathrm{dt}}
$$

donde $\frac{\mathrm{dS}}{\mathrm{dt}}$ es el cambio de agua almacenada en el suelo (g), $\frac{\mathrm{dP}}{\mathrm{dt}}$ es la cantidad de agua que ingresa por lluvia (g), $\frac{\mathrm{dIR}}{\mathrm{dt}}$ es la cantidad que ingresa por riego (g), $\frac{\mathrm{dD}}{\mathrm{dt}}$ es la cantidad de agua drenada más allá de la zona de raíces (g), $\frac{\mathrm{dET}}{\mathrm{dt}}$ es la cantidad de agua pérdida por la evapotranspiración del cultivo (g), y $\frac{\mathrm{dSr}}{\mathrm{dt}}$ es el agua pérdida por escurrimiento superficial (g), todo los parámetros evaluados con respecto al tiempo. 
Únicamente, los eventos de lluvia fueron analizados para conocer la velocidad de entrada de agua dentro del suelo. Las lluvias tienen una duración más larga de aplicación y por lo tanto dan una mejor exactitud de los resultados. Las variables de riego y evapotranspiración no fueron consideradas durante los eventos de lluvia, excepto en la determinación de los contenidos de humedad, ya que hay lapsos de tiempo sin lluvia (Hannes et al., 2015; Peters et al., 2014), así que la Ecuación 12 fue reformulada a la ecuación 13:

$$
\frac{\mathrm{dS}}{\mathrm{dt}}=\frac{\mathrm{dP}}{\mathrm{dt}}-\frac{\mathrm{dD}}{\mathrm{dt}}-\frac{\mathrm{dSr}}{\mathrm{dt}}
$$

El escurrimiento fue despreciado debido a que no fue observado en este experimento. Las variables conocidas del experimento son las masas acumuladas de los recipiente del suelo (S) y depósito de drenaje (D), así que el flujo de ambos recipientes es determinado por el incremento de la masa (m) del recipiente sobre el tiempo (Hannes et al., 2015), como la Ecuación 14:

$$
\frac{\mathrm{dS}_{\text {lis }}}{\mathrm{dt}} \text { o } \frac{\mathrm{dD}_{\text {lis }}}{\mathrm{dt}}=\frac{\mathrm{m}_{\mathrm{i}+1}-\mathrm{m}_{\mathrm{i}}}{\mathrm{t}_{\mathrm{i}+1}-\mathrm{t}_{\mathrm{i}}}
$$

donde $\frac{\mathrm{dD}_{\text {lis }}}{\mathrm{dt}}$ es el flujo de agua del depósito de drenaje sobre el tiempo y $\frac{\mathrm{d} \mathrm{S}_{\text {lis }}}{\mathrm{dt}}$ es el flujo de agua en recipiente de suelo sobre el tiempo.

La lluvia es calculada a partir de la metodología reportada por Haselow et al. (2019), Hannes et al. (2015), Gebler et al. (2015) y Peters et al. (2014), cuando se cumple la condición de $\frac{d S_{\text {lis }}}{d t}+\frac{d D_{\text {lis }}}{d t}>0$; por lo que la Ecuación 15 fue usada:

$$
\frac{\mathrm{dP}}{\mathrm{dt}}=\frac{\mathrm{dS}_{\text {lis }}}{\mathrm{dt}}+\frac{\mathrm{dD}_{\text {lis }}}{\mathrm{dt}}
$$




\subsubsection{Cálculo de la velocidad de infiltración del agua en el suelo}

La infiltración del agua tiene un comportamiento vertical descendente en el suelo dentro del lisímetro (Ruiz-Peñalver et al., 2015) y en condiciones libres de drenaje (Herrada et al., 2014). Cuando el contenido de humedad del suelo está por debajo del contenido de agua a capacidad de campo, el aumento del agua almacenada en el recipiente del suelo representa la velocidad de infiltración $\frac{\mathrm{d} \mathrm{I}}{\mathrm{dt}} \sin$ la componente de drenaje. La velocidad de infiltración fue entonces representada con la Ecuación 16, la cual fue llamada Enfoque 1 (Wang and Chu, 2020):

$$
\frac{\mathrm{dI}}{\mathrm{dt}}=\frac{\mathrm{dS}_{\mathrm{lis}}}{\mathrm{dt}}
$$

Sin embargo, una vez alcanzado el contenido de agua a capacidad de campo, el suelo no es capaz de almacenar más agua y por lo tanto comenzará a drenar agua (Wang and Chu, 2020). En este sentido, la suma del agua almacenada y drenada representa la velocidad de infiltración del agua en el perfil del suelo; el modelo que representa la $\frac{\mathrm{dI}}{\mathrm{dt}}$ es representado en la Ecuación 17 el cual es llamado Enfoque 2:

$$
\frac{\mathrm{dI}}{\mathrm{dt}}=\frac{\mathrm{dS}_{\mathrm{lis}}}{\mathrm{dt}}+\frac{\mathrm{dD}_{\mathrm{lis}}}{\mathrm{dt}}
$$

Ambos enfoques son válidos si el contenido de agua a saturación no es alcanzado y si la intensidad de lluvia no sobrepasa la capacidad de infiltración básica del suelo. En este experimento el suelo nunca llego a sobrepasar a ambos, por lo que no hubo escorrentía superficial. Estas ecuaciones cumplen con el principio de la conservación de la masa y ecuación de continuidad.

Los flujos y velocidades de infiltración resultantes representan el área del lisímetro $\left(0.54 \mathrm{~m}^{2}\right)$, es decir $1 \mathrm{~kg} \cdot \mathrm{min}^{-1}$ del lisímetro es igual a $1.85 \mathrm{~kg} \cdot \mathrm{min}^{-1} \mathrm{o} 1.85 \mathrm{~mm} \cdot \mathrm{min}^{-1} \mathrm{de} 1 \mathrm{~m}^{2}$ de superficie, asumiendo que un $1 \mathrm{~L}$ de agua es igual a $1 \mathrm{~kg}$ de masa de agua y que $1 \mathrm{~mm}$ es igual a $1 \mathrm{~L} \cdot \mathrm{m}^{-2}$ (Haselow et al., 2019). 
Los eventos de riego no fueron analizados porque su tiempo y volumen de aplicación fueron pequeños y no permiten la visualización de la curva de infiltración; la velocidad de infiltración fue constante a través de los dos o tres horas de riego.

\subsubsection{Estimación del contenido de agua en el suelo}

El contenido de agua en el perfil del suelo dentro del recipiente del suelo fue determinado por el método gravimétrico, la masa del suelo húmedo era conocida en cualquier instante a partir de los datos registrados del recipiente del suelo. Una vez, que el valor del contenido de agua gravimétrico era conocido fue convertido a un valor volumétrico $\left(\theta_{\mathrm{v}}\right)$ con la Ecuación 18:

$$
\theta_{\mathrm{v}}=\left(\frac{\mathrm{m}_{\mathrm{i}}-\mathrm{m}_{\text {seca }}}{\mathrm{m}_{\text {seca }}}\right) \cdot \rho_{\mathrm{b}}
$$

donde $\theta_{\mathrm{v}}$ es el contenido volumétrico del instante $\mathrm{i}\left(\mathrm{cm}^{3} \cdot \mathrm{cm}^{-3}\right), \rho_{\mathrm{b}}$ es la densidad aparente del suelo, $\mathrm{m}_{\mathrm{i}}$ es la masa húmeda del suelo dentro del recipiente del suelo en el instante i (g) $\mathrm{y}_{\text {seca }}$ es la masa seca del suelo dentro del recipiente del suelo. Este último valor es inferido de la densidad aparente del suelo y el volumen total del recipiente del suelo $\left(0.161 \mathrm{~m}^{3}\right)$ (Villalobos et al., 2002). La masa húmeda en el instante i representa la suma de suelo y agua; la masa de la estructura del recipiente no es considerada ya que este fue tarado a cero antes de insertar el suelo dentro del recipiente.

El contenido de humedad a capacidad de campo $\left(\theta_{\mathrm{CC}}\right)$ fue establecida dos o tres días después de los eventos de lluvia, cuando el suelo paro de drenar y el recipiente del suelo tuvo un valor constante, indicando que el suelo ya era capaz de retener el agua (Kirkham, 2010). La evapotranspiración fue calculada por su efecto en la redistribución del agua en el suelo, la cual fue obtenida usando el método de balance de masas. (Ecuación 12), teniendo en cuenta que la evapotranspiración y la lluvia no ocurren al mismo tiempo. 


\subsubsection{Validación}

Un total de 12 eventos de lluvia del 2017 fueron analizados desde el 8 de febrero hasta el 30 de mayo, los cuales fueron i) 13 de febrero, ii) 18 de febrero, iii) 19 de febrero, iv) 24 de febrero, v) 13 de marzo, vi) 14 de marzo, vii)27 de abril, viii) 28 de abril, ix) 29 de abril, x) 30 de abril, xi) 10 de mayo y xii) 30 de mayo. En el caso del Enfoque 2, solamente fueron usados los eventos v) hasta $\mathrm{x}$ ) ya que en esos eventos ocurre el drenaje.

\subsubsection{Modelos de calibración}

Los resultados de la velocidad de infiltración $\left(\frac{\mathrm{d} I}{\mathrm{dt}}\right)$ del suelo fueron usados para calibrar modelos de infiltración empíricos y físicos de autores previamente mencionados. Uno de ellos es el modelo Kostiakov, el cual puede ser representado por la Ecuación 19:

$$
\frac{d I}{d t}=a \cdot b \cdot t^{b-1}
$$

donde t es el tiempo de infiltración y a y b son constantes empíricas (Ali, 2010; Fok, 1986; Hartley, 1992; Kirkham, 2010; Lewis, 1937). Esta ecuación tiene una condición límite, $\lim _{\mathrm{t} \rightarrow \infty} \mathrm{dI} / \mathrm{dt}=0$ (Haverkamp et al., 1988; Philip, 1957). Los dos parámetros empíricos fueron determinados a partir de un ajuste iterativo de datos obtenidos experimentalmente con nuestros dos enfoques (Hartley, 1992).

El parámetro b de la ecuación de Kostiakov está limitado a ser más grande que cero y menor a uno; por lo que el valor inicial de la velocidad de infiltración tiende a ser infinito y una velocidad básica a cero en un tiempo infinito. En eventos de riego o lluvias grandes, Lewis, (1937) observó que la velocidad de infiltración tiende a un valor constante antes del final del riego o lluvia. Por consiguiente, la ecuación de Kostiakov en los eventos largos usualmente sobreestima la infiltración (Haghiabi et al., 2011; Smerdon et al., 1988), para solucionar esto una constante fue añadida a la Ecuación 20 para representar la velocidad de infiltración básica, resultando en la ecuación de Kostiakov Modificada: 


$$
\frac{d I}{d t}=a \cdot b \cdot t^{b-1}+c
$$

donde c es una constante empírica que representa la velocidad de infiltración básica (Ali, 2010; Furman et al., 2006; Strelkoff et al., 2009). Esta ecuación también es conocida como Kostiakov-Lewis o modelo de Mezencev. Su condición limite es $\lim _{\mathrm{t} \rightarrow \infty} \mathrm{dI} / \mathrm{dt}=\mathrm{c}$ (Haverkamp et al., 1988).

El modelo de Philip para la velocidad de infiltración de un medio poroso homogéneo es una simplificación del modelo de Richards. El primer término de la ecuación establece la absorción capilar del agua dentro del suelo (S) y el segundo término representa una serie truncada de un pequeño tiempo de encharcamiento (A), representado en la Ecuación 21:

$$
\frac{\mathrm{dI}}{\mathrm{dt}}=\frac{1}{2} \cdot \mathrm{S} \cdot \mathrm{t}^{-1 / 2}+\mathrm{A}
$$

donde S es la sorptividad y A es un valor constante (Philip, 1957; Smerdon et al., 1988). Su condición límite es $\lim _{\mathrm{t} \rightarrow \infty} \mathrm{dI} / \mathrm{dt}=\mathrm{A}$ (Haverkamp et al., 1988).

Horton establece un modelo basado en el comportamiento natural de la ley de exponencial inversa, es decir la velocidad de infiltración que disminuye exponencialmente a un valor contante y es proporcional al volumen infiltrado como sigue:

$$
\frac{d I}{d t}=V_{f}+\left(V_{0}-V_{f}\right) e^{-\beta \cdot t}
$$

donde $V_{f}$ es la velocidad de infiltración final o básica. $V_{0}$ es ala velocidad de infiltración inicial y $\beta$ es una constante empírica de cambio desde velocidad inicial a final (Horton, 1941). La condición limite es $\lim _{\mathrm{t} \rightarrow \infty} \mathrm{dI} / \mathrm{dt} \geq 0$ (Philip, 1957).

Cuando comparamos los cuatro modelos anteriormente descritos puede ser observado que los parámetros del modelo de Kostiakov no tienen significado físico y no es apto para largos tiempos ya que se pierde su representatividad y exactitud de la velocidad de infiltración del agua en el suelo, además para un tiempo igual a cero, la velocidad de 
infiltración parece indeterminada. En el modelo de Kostiakov Modificado una constante fue añadida para representar la velocidad básica de en largos tiempos de infiltración. En el caso del modelo de Horton, la infiltración inicial es definida con un valor infinito y su modelo describe el comportamiento de la velocidad como una curva inversa exponencial. Finalmente, Philip describe la velocidad de infiltración cuando hay una pequeña cantidad de encharcamiento y sus parámetros tienen significado físico para el comportamiento del suelo cuando permite la entrada de agua.

Las estadísticas usadas para evaluar la exactitud de la estimación de la velocidad de infiltración del agua en el suelo usando el lisímetro de pesada y los modelos mencionados arriba fueron: desviación estándar (s), raíz del error cuadrático medio (RMSE) y el coeficiente de determinación $\left(\mathrm{R}^{2}\right)$ a través de las siguientes ecuaciones:

$$
\begin{gathered}
s=\sqrt{\frac{1}{n-1} \sum_{i=1}^{n}\left(Y_{i}-\widehat{Y}_{i}\right)^{2}} \\
\text { RMSE }=\sqrt{\frac{1}{n} \sum_{i=1}^{n}\left(Y_{i}-\widehat{Y}_{i}\right)^{2}} \\
R^{2}=1-\frac{\sum_{i=1}^{n}\left(\widehat{Y}_{i}-\bar{y}\right)^{2}}{\sum_{i=1}^{n}\left(Y_{i}-\bar{y}\right)^{2}}
\end{gathered}
$$

donde $n$ es el número de datos, $Y_{i}$ es el valor medido en $i, \widehat{Y}_{i}$ es el valor estimado en $i$ y $\bar{y}$ es la media de los valores medidos (Wackerly et al., 2010). 


\subsection{Evapotranspiración del pimiento y coeficiente de cultivo}

\subsection{1. Área de estudio}

El experimento fue conducido durante 2019 y 2020 en una parcela comercial de pimiento (Capsicum annuum L. var. Maestral) localizada en San Javier en la Región de Murcia en el sureste de España (Fig. 11), con coordenadas geográficas 3751'11.80" N, 049'50.00" E y una altitud de 15 m.s.n.m. (metros sobre el nivel del mar). La parcela experimental está a campo abierto y con área circundante dominada por invernaderos, la parcela es resaltada en rojo en la Fig. 11b. El clima predominante de la zona de estudio ha sido identificado como subdesierto Mediterráneo, con temperatura máxima y mínima de $36.6^{\circ} \mathrm{C}$ y $12.9^{\circ} \mathrm{C}$, respectivamente, y una lluvia anual promedio de $313 \mathrm{~mm}$ (EscarabajalHenarejos et al., 2015).

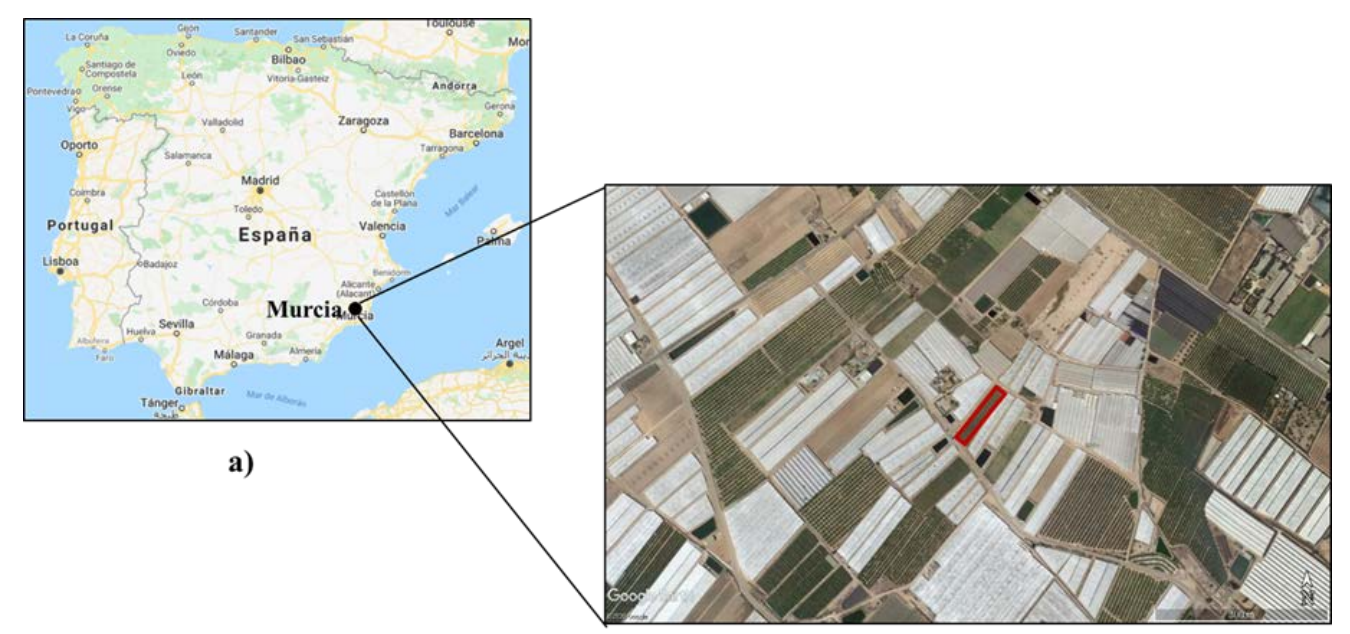

b)

Figura 11. Área de estudio, a) Región de Murcia y b) localización de la parcela experimental.

\subsubsection{Descripción del lisímetro de pesada compacto}

El lisímetro de pesada usado en esta investigación es el mismo descrito en el apartado 3.1.2. El dispositivo fue instalado en el centro de la parcela, en un agujero excavado 
considerando las dimensiones del lisímetro, evitando los bordes debido a que es necesario que este rodeado por la misma vegetación para obtener mediciones confiables (Aboukhaled et al., 1982). El suelo extraído en la excavación fue colocado en el recipiente superior del lisímetro, tratando de mantener el suelo inalterado. Una caracterización hidrodinámica del suelo arcilloso fue realizado basado en la metodología propuesta por USDA (Staff, 1951) con una densidad aparente de $1.38 \mathrm{~g} \cdot \mathrm{cm}^{-3}$. El sistema de riego usado fue goteo, con emisores colocados a cada $25 \mathrm{~cm}$ y una tasa de aplicación de $2.2 \mathrm{~L} \cdot \mathrm{h}^{-1}$ (Fig. 12).

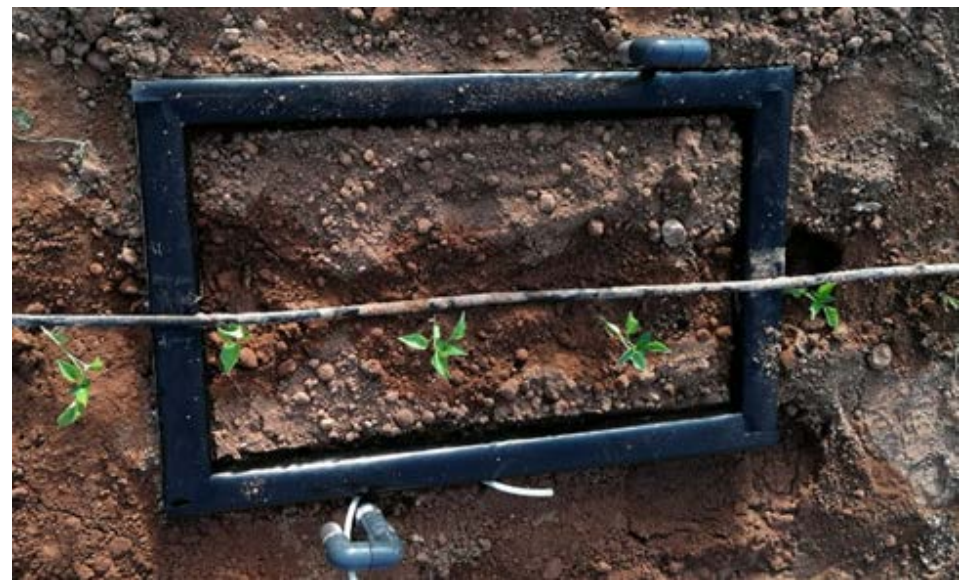

Figura 12. Vista superior del lisímetro de pesada compacto durante la experimentación.

\subsubsection{Manejo del cultivo}

El cultivo del pimiento, previamente sembrado bajo condiciones de invernadero, fue trasplantado con una altura de $10 \mathrm{~cm}$. El marco de plantación fue de $1 \mathrm{~m}$ entre líneas y $0.33 \mathrm{~m}$ entre plantas, dejando un total de tres plantas y tres goteros dentro del área del lisímetro como se observa en la Fig. 12. El ciclo vegetativo hasta la cosecha fue de 195 días. El esquema de riego fue programado para compensar las pérdidas por ET $_{\mathrm{C}}$ calculadas con el lisímetro y con una eficiencia de aplicación del 91\%.

La dosis de fertilización fue aplicada con el agua de riego acorde a las recomendaciones del Ministerio de Agricultura Pesca y Alimentación (2020); mientras tanto, 
las malas hierbas y las plagas se manejaban de acuerdo con las prácticas comunes en la zona agrícola.

\subsubsection{Determinación de la Evapotranspiración y coeficientes de cultivo}

El cálculo de la evapotranspiración comenzó el día del trasplante del cultivo. Luego, mediante la aplicación del riego, el suelo alcanzó la capacidad de campo, el valor inicial de recipiente del suelo representa la masa del suelo más la masa de agua más la masa de tres plantas. Con el paso del tiempo, si el suelo no recibía agua, la masa del recipiente disminuía debido a la $\mathrm{ET}_{\mathrm{C}}$. Cuando se aplicaba el riego o se producían precipitaciones, la masa del recipiente del suelo aumentaba rápidamente; si se excedía la capacidad de campo del suelo, éste comenzaba a drenar o descargar el exceso de agua de la parte inferior del recipiente del suelo, lo que se reflejaba en el aumento de la masa del depósito de drenaje. Una vez que se detuvo el riego o las precipitaciones, el suelo volvía a perder agua debido a la $\mathrm{ET}_{\mathrm{C}}$.

A partir del comportamiento operacional del lisímetro descrito anteriormente, se calculó la evapotranspiración diaria del cultivo aplicando la ecuación de balance hídrico (Miranda et al., 2006) y las condiciones establecidas en el trabajo de Peters et al. (2014), según las ecuaciones (26) y (27):

$$
\mathrm{ET}_{\mathrm{C}_{\mathrm{LYS}}}=\mathrm{I}-\Delta \mathrm{DD}-\Delta \mathrm{RS}
$$

En condiciones de ausencia de agua de lluvia o de riego, la ecuación (26) quedaría como sigue:

$$
\mathrm{ET}_{\mathrm{C}_{\mathrm{LYS}}}=-\Delta \mathrm{RS}-\Delta \mathrm{DD}
$$

donde I es la profundidad de riego (mm), $\Delta$ RS es la variación de masa en el recipiente de del suelo (mm), y $\Delta \mathrm{DD}$ es el aumento de masa en el depósito de drenaje (mm). 
A partir de los datos obtenidos con el lisímetro, se calcularon los valores diarios del coeficiente de cultivo $\left(\mathrm{K}_{\mathrm{C}_{\text {lys }}}\right)$ mediante la Ecuación 28:

$$
\mathrm{K}_{\mathrm{Clys}_{\mathrm{lys}}}=\frac{\mathrm{ET}_{\mathrm{C}_{\mathrm{LYS}}}}{\mathrm{ET}_{\mathrm{O}}}
$$

donde $\mathrm{ET}_{\mathrm{O}}$ es la evapotranspiración de referencia estimada por el método FAO Penman-Monteith (Allen et al., 1998), considerando los registros climáticos generados en una estación meteorológica automática construida con un registrador de datos (datalogger modelo CR10X, Campbell Scientific, Logan, UT, USA), un piranómetro (modelo CM14, KIPP\&ZONEN Delft), sensores de temperatura y humedad relativa colocados en $1.5 \mathrm{~m}$ sobre el nivel del suelo (sonda MP100, Campbell Scientific, Logan, UT, USA), un anemómetro y una anemoveleta a $2 \mathrm{~m}$ de altura (modelos A100R y W200P, respectivamente, de Vector Instruments Ltd., Rhyl, North Wales, UK), y un pluviómetro (modelo ARG100, Campbell Scientific, Logan, UT, USA). La estación meteorológica automática se instaló junto al lisímetro de pesada. La duración de las etapas fenológicas se definieron de acuerdo con las recomendaciones de Allen et al. (1998).

El valor de $\mathrm{ET}_{\mathrm{C}}$ determinado con el método $\mathrm{FAO}-56$ se comparó con el $\mathrm{ET}_{\mathrm{C}_{\mathrm{LYS}}}$ obtenido con el lisímetro, el modelo FAO-56 (Allen et al., 1998) es el siguiente:

$$
\mathrm{ET}_{\mathrm{C}_{\mathrm{FAO}}}=\mathrm{ET}_{\mathrm{O}} \cdot \mathrm{K}_{\mathrm{C}_{\mathrm{FAO}}}
$$

donde $\mathrm{K}_{\mathrm{C}_{\mathrm{FAO}}}$ hace referencia a los valores propuestos por la FAO en el Manual 56 para el pimiento para las diferentes etapas fenológicas del cultivo.

A partir de la temperatura media del aire obtenida con la estación meteorológica, se determinaron las unidades térmicas de cultivo (TU) para todo el ciclo vegetativo (MartínezCob, 2008):

$$
\text { Si } \mathrm{T}_{\mathrm{a}}>\mathrm{T}_{\mathrm{b}} \text { : }
$$




$$
\mathrm{TU}_{\mathrm{i}}=\left(\mathrm{T}_{\mathrm{a}}-\mathrm{T}_{\mathrm{b}}\right)_{\mathrm{i}}
$$

If $\mathrm{T}_{\mathrm{a}} \leq \mathrm{T}_{\mathrm{b}}$ :

$$
\mathrm{TU}_{\mathrm{i}}=\mathrm{TU}_{\mathrm{i}-1}
$$

donde $\mathrm{TU}_{\mathrm{i}}$ es la unidad térmica del día $i$ en ${ }^{\circ} \mathrm{C}, \mathrm{TU}_{\mathrm{i}-1}$ es la unidad térmica del día $\mathrm{i}-1$ en ${ }^{\circ} \mathrm{C}$, $\mathrm{T}_{\mathrm{b}}$ es la temperatura basal o mínima a la que puede desarrollarse el pimiento, la cual es de $10{ }^{\circ} \mathrm{C}$ (Vidal, 2011) y $T_{a}$ es la temperatura media del aire en ${ }^{\circ} \mathrm{C}$ del día i.

Los valores TU se convirtieron en unidades térmicas de fracción acumulada (FTU):

$$
\mathrm{FTU}_{\mathrm{i}}=\frac{\mathrm{TU}_{\mathrm{i}}}{\Sigma \mathrm{TU}_{\mathrm{i}}}
$$

donde $\Sigma \mathrm{TU}_{\mathrm{i}}$ es la suma total de las TU. Para un óptimo crecimiento del pimiento Vidal (2011) propone un valor $\Sigma$ TU de $2020 \pm 220^{\circ} \mathrm{C}$. En este experimento, el total de unidades térmicas acumuladas para las temporadas 2019 y 2020 fue de 1860 y $1904{ }^{\circ} \mathrm{C}$, respectivamente. Se obtuvo una relación entre los resultados de la FTU y el coeficiente de cultivo del lisímetro utilizando el algoritmo de los mínimos cuadrados (Verma, 2016).

Se utilizaron diferentes estadísticos para comparar los resultados obtenidos de las diferentes metodologías, como el error medio de estimación (MEE), la raíz del error cuadrático medio (RMSE), el error cuadrático medio sistemático ( $\mathrm{MSE}_{\mathrm{S}}$ ), el coeficiente de determinación $\left(\mathrm{R}^{2}\right.$ ) y el índice de acuerdo (IA):

$$
\begin{aligned}
\text { MEE } & =\frac{1}{n} \sum_{i=1}^{n} Y_{i}-\widehat{Y}_{i} \\
M_{S E} & =\frac{1}{n} \sum_{i=1}^{n}\left(\widehat{Y}_{i}-Y_{i}\right)^{2}
\end{aligned}
$$




$$
I A=1-\frac{\sum_{i=1}^{n}\left(Y_{i}-\widehat{Y}_{i}\right)^{2}}{\sum_{i=1}^{n}\left(|\widehat{Y}-\bar{y}|+\left|Y_{i}-\bar{y}\right|\right)^{2}}
$$

donde $\mathrm{n}$ es el número de datos, $\mathrm{Y}_{\mathrm{i}}$ es el valor medido de $\mathrm{i}, \widehat{\mathrm{Y}}_{\mathrm{i}}$ es el valor estimado en la regresión del día i o el valor a ser comparado y y es la media de los valores medidos del día i (Wackerly et al., 2010; Willmott et al., 1985).

\subsubsection{Productividad del agua}

Según la metodología propuesta por Playán and Mateos, (2006), la productividad del agua se determinó como la relación entre la producción obtenida y el volumen consumido por el cultivo, expresado en $\mathrm{kg} \cdot \mathrm{m}^{-3}$, el rendimiento del cultivo por superficie cultivada en $\mathrm{t} \cdot \mathrm{ha}^{-1} \mathrm{y}$ los beneficios económicos por superficie cultivada, en $€ \cdot h \mathrm{~h}^{-1}$. 


\section{Resultados y Discusión}

\subsection{Infiltración del agua en el suelo}

La Fig. 13 muestra las curvas de masa de los dos recipientes de cultivo del lisímetro de pesada compacto en el intervalo de tiempo analizado. Los datos iniciales reportados por el recipiente del suelo indica la masa de las partículas del suelo más el contenido del agua. Por consiguiente, las variaciones de masa registradas después de este punto inicial indican las variaciones del contenido de agua en el suelo.

Los aportes de la lluvia y riego se pueden diferenciar por el tipo de aumento que presentan en la curva del recipiente del suelo (línea azul de la Fig. 13). El riego tiene un aumento muy vertiginoso en poco tiempo, mientras que la lluvia tiene un crecimiento discontinuo en el tiempo, en cambio, los descensos pueden representar las pérdidas por evapotranspiración, las pérdidas por drenaje o ambas.

En el caso de los datos del recipiente de drenaje (línea roja de la Fig. 13), la subida de la curva representa que el drenaje ha comenzado y los descensos repentinos indican el vaciado del recipiente. Como se muestra en la Fig. 13, únicamente dos eventos de drenaje fueron registrados por el recipiente el 13-14 de marzo y el 28-30 de abril. El recipiente de 
drenaje fue programado para abrir su válvula a los $3000 \mathrm{~g}$ de almacenamiento y descarga (para evitar dañar la celda de carga); por lo tanto, la curva del recipiente de drenaje en la Fig. 13 muestra masas acumuladas inferiores a $4000 \mathrm{~g}$.

El vaciado es casi instantáneo, toma menos de 3 minutos y no se pierde ninguna gota ya que la válvula debajo del recipiente del suelo es cerrada. El evento de marzo causó más drenaje que el de abril, en menos tiempo de lluvia. Esto indica que la intensidad de la lluvia del evento de marzo fue mayor que la del evento de abril, más agua cayó en menos tiempo. Los siete eventos de lluvia entre febrero y marzo contribuyeron a que el primer evento de drenaje ocurriera el 14 de marzo. Mientras tanto, los cinco eventos de lluvia ocurridos entre abril y mayo causaron el segundo evento de drenaje en el día 28-30 de abril.

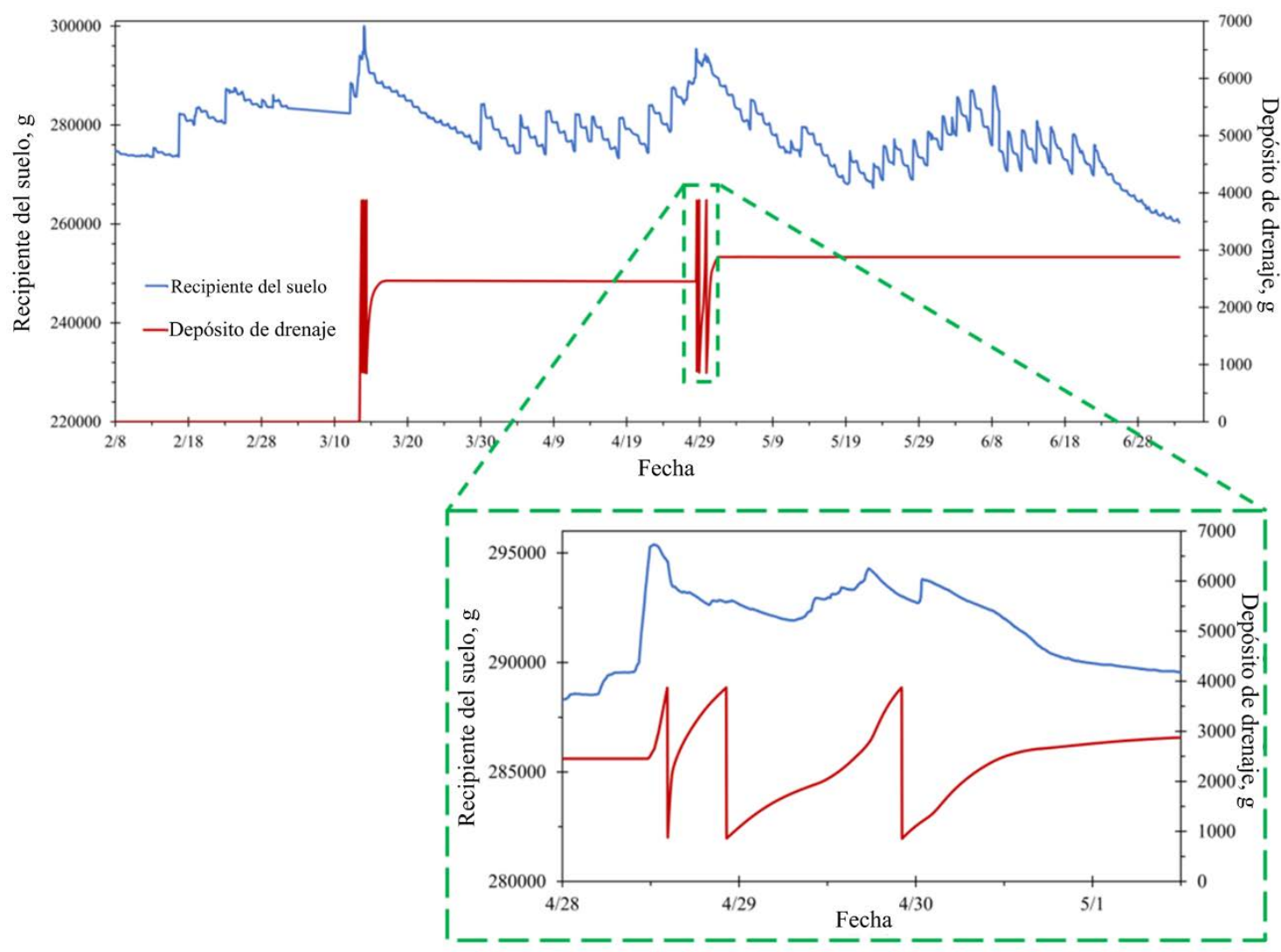

Figura 13. Variaciones de masa de los dos recipientes del lisímetro de pesada compacto durante el 2017. 


\subsubsection{Estimación de la entrada de la lluvia}

Los días lluviosos totales analizados fueron 12 y fueron determinados con la Ecuación 15, representando 11 eventos de lluvia. La Tabla 1 muestra los datos de lluvia registrados por día en el lisímetro de pesada y los registrados en un pluviómetro de la estación meteorológica perteneciente al Instituto Agronómico Provincial (ITAP) instalada a $800 \mathrm{~m}$ del lisímetro. La lluvia calculada con el lisímetro para el primer día analizado tiene una diferencia de 0.05 mm con el pluviómetro. Las diferencias encontradas pueden ser explicadas por el comportamiento espacial de la variabilidad de la lluvia (Camarasa Belmonte et al., 2010) en una región, la cual tiene un impacto más grande en las zonas semiáridas como la de este estudio. Hay una diferencia promedio de $1.59 \mathrm{~mm}$ entre los dos dispositivos.

Tabla 1. Datos de lluvia de los días analizados por los diferentes dispositivos

\begin{tabular}{crr}
\hline & \multicolumn{3}{c}{ Lluvia registrada por día } \\
\cline { 2 - 3 } Fecha & $\begin{array}{c}\text { Lisímetro de pesada } \\
(\mathrm{mm})\end{array}$ & $\begin{array}{c}\text { Estación meteorológica } \\
\text { ITAP }(\mathrm{mm})\end{array}$ \\
\hline $13 / 02$ & 3.55 & 3.60 \\
$18 / 02$ & 3.16 & 3.30 \\
$19 / 02$ & 2.76 & 2.90 \\
$24 / 02$ & 1.30 & 1.00 \\
$13 / 03$ & 36.77 & 26.70 \\
$14 / 03$ & 28.53 & 22.30 \\
$27 / 04$ & 7.04 & 6.40 \\
$28 / 04$ & 7.06 & 5.90 \\
$29 / 04$ & 8.16 & 8.30 \\
$30 / 04$ & 2.44 & 1.6 \\
$10 / 05$ & 1.01 & 1.10 \\
$30 / 05$ & 2.11 & 1.60 \\
\hline
\end{tabular}




\subsubsection{Estimación del contenido de agua en el suelo}

La Fig. 14 muestra el comportamiento de la lluvia y su relación con el contenido de agua del suelo, se observa que cuando la lluvia cae, hay un aumento de masa, y cuando el suelo alcanza la capacidad de campo y la lluvia continúa, hay drenaje; cuando hay una caída en la curva de humedad, el drenaje aumenta. Dos eventos de drenaje fueron causados con los eventos de lluvia de 13 y 14 de marzo, 28 de abril, 29 de abril, y 30 de abril así que con estos eventos fue posible determinar la capacidad de campo del suelo $\left(\theta_{\mathrm{FC}}\right)$ basado en su definición y con el método gravimétrico (Ecuación 18).

Al trazar la curva de contenido de agua con la del depósito de drenaje fue posible determinar el momento en que se alcanzó $\theta_{\mathrm{FC}}$. En ambos meses, el $\theta_{\mathrm{FC}}$ se estableció cuando la curva del depósito de drenaje se hizo constante, indicando que el suelo había dejado de drenar y que no había ninguna variación en el lisímetro de peso. Se trazó una línea horizontal sobre el contenido de agua en el momento en que no se incrementó la masa del depósito de drenaje, lo que indica que se alcanzó el $\theta_{\mathrm{FC}}$ y se estableció dos días después del comienzo de la lluvia en $0.33 \mathrm{~cm}^{3} \cdot \mathrm{cm}^{-3}$ (Fig. 14).

a)
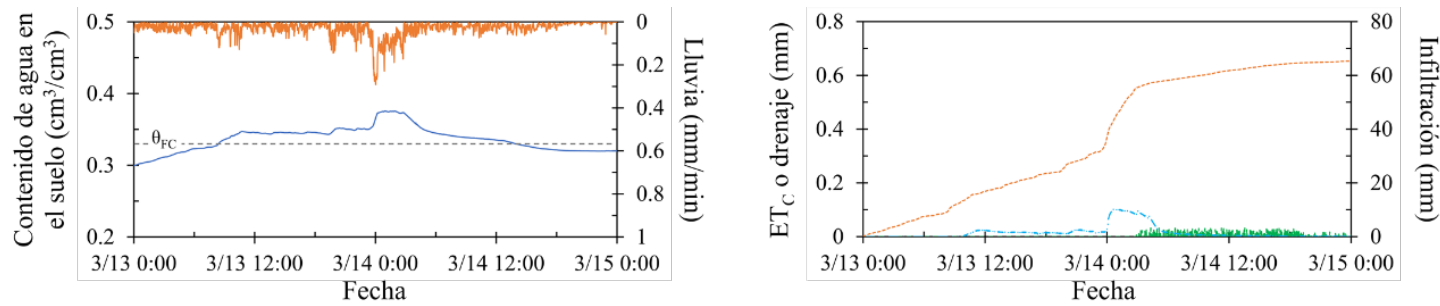

b)
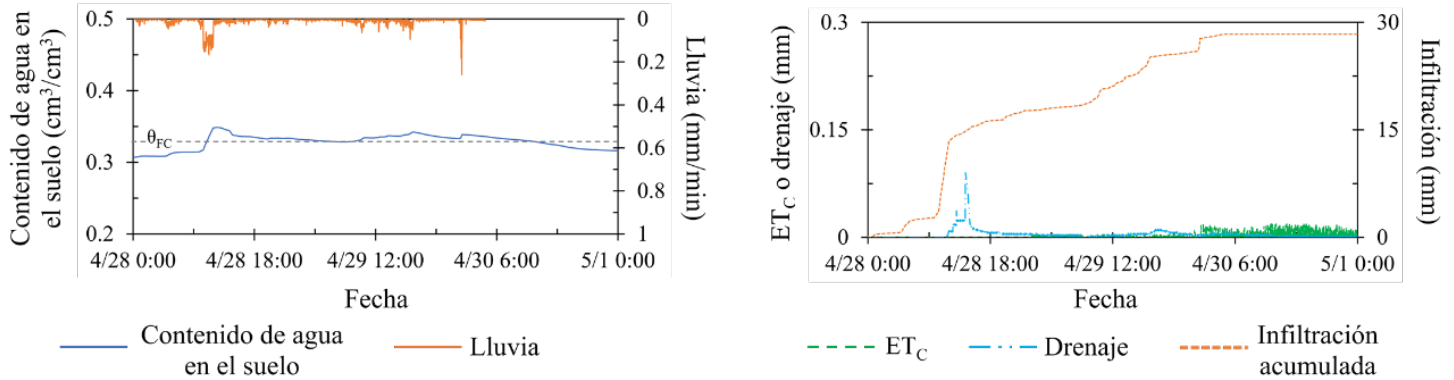

Figura 14. Contenido de agua en el suelo y variable hidrológicas obtenidas con el lisímetro de pesada compacto, a) Marzo y b) Abril. 
Tabla 2. Balance hídrico de los días involucrados en la determinación de la $\theta_{F C}$.

\begin{tabular}{ccccc}
\hline Fecha & $d I R / d t, \mathrm{~mm}$ & $d P / d t, \mathrm{~mm}$ & $d D / d t, \mathrm{~mm}$ & $d E T / d t, \mathrm{~mm}$ \\
\hline $13 / 03$ & 0.00 & 36.77 & 14.04 & 0.00 \\
$14 / 03$ & 0.00 & 28.53 & 29.57 & 5.41 \\
$28 / 04$ & 10.62 & 5.90 & 8.65 & 0.00 \\
$29 / 04$ & 0.00 & 8.30 & 5.65 & 0.65 \\
$30 / 04$ & 0.00 & 1.60 & 3.05 & 4.94 \\
\hline
\end{tabular}

Como el $\theta_{\mathrm{FC}}$ se estableció días después de los eventos de lluvia, la evapotranspiración $\left(\frac{\mathrm{dET}}{\mathrm{dt}}\right)$ estaba presente, por lo que fue calculada como se indica en los trabajos de Hannes et al. (2015), Haselow et al. (2019), y Peters et al. (2014), es decir con la Ecuaciones 26 y 27. La Tabla 2 tiene el balance hídrico de los días involucrados en el cálculo del $\theta_{\mathrm{FC}} \cdot \frac{\mathrm{dET}}{\mathrm{dt}}$ es una variable que resta la humedad de la superficie del suelo, en los primeros días de cada evento representa el $0 \%$ de las pérdidas de agua y de las precipitaciones; aumentando a medida que la lluvia disminuye a lo largo de los días, de modo que en el segundo día $\frac{\mathrm{dET}}{\mathrm{dt}}$ representa el $15.47 \%$ y el $10.32 \%$ de las pérdidas de agua y el $18.96 \%$ y el $7.83 \%$ de las precipitaciones. El total de las pérdidas de $\frac{\mathrm{dET}}{\mathrm{dt}}$ para todos los días de cada evento utilizado para el cálculo de $\theta_{\mathrm{FC}}$ representa el 11.04\% para el primero (Fig. 14a) y el 24.37\% para el segundo (Fig. 14b). $\frac{\mathrm{dET}}{\mathrm{dt}}$ representa el $8.28 \%$ de la lluvia del primer evento (Fig. 14a) y el 35.38\% del segundo evento (Fig. 14b).

La Tabla 3 informa los valores del contenido de humedad a capacidad de campo obtenidos y algunos de la literatura para un suelo franco limoso. Los valores con menor desviación estándar con el resultado del lisímetro de pesada fueron el valor obtenido en el laboratorio en esta investigación y el valor máximo reportado por Assi et al. (2019) con $0.014 \mathrm{~cm}^{3} \cdot \mathrm{cm}^{-3}$. Seguido por los valores reportados por Allen et al. (1998) en gráficos y tablas y que son obtenidos usando los datos de la textura del suelo $\left(\theta_{\text {saturación }}, \theta_{\mathrm{FC}} \mathrm{y}\right.$ $\theta_{\text {punto de marchitamiento permanente }}$ ) con $0.02 \mathrm{~cm}^{3} \cdot \mathrm{cm}^{-3}$, el cual tiene una buena concordancia con los resultados obtenidos en el laboratorio y el lisímetro. 
Tabla 3. Comparación del contenido de agua a capacidad de campo obtenido en el suelo con los valores de la literatura.

\begin{tabular}{ccc}
\hline$\theta_{\mathrm{FC}}, \mathrm{cm}^{3} \cdot \mathrm{cm}^{-3}$ & \multicolumn{2}{c}{ Método de estimación e investigadores } \\
\hline 0.33 & Lisímetro de pesada & \\
0.35 & Laboratorio & \\
$0.22-0.36$ & FAO & (Allen et al., 1998) \\
$0.30-0.31$ & Pedo-estructura & (Assi et al., 2019) \\
0.244 & Gravimétrico & (Porta-Casanellas and \\
& & Lopez-Acevedo, 2005) \\
\hline
\end{tabular}

\subsubsection{Velocidad de infiltración del agua en el suelo}

Se analizaron siete lluvias con el Enfoque 1 cuando el suelo estaba por debajo de la capacidad de campo (13, 18, 19 y 24 de febrero, 27 de abril y 10 y 30 de mayo), de modo que las variaciones positivas reportadas en el recipiente del suelo indicaban la velocidad de infiltración de agua. Según USDA et al. (1999) y Evanylo and McGuinn, (2000) la mejor manera de determinar la velocidad de infiltración es cuando el suelo está cerca de la capacidad de campo; cuatro eventos de cinco días de lluvia tuvieron drenaje debido a que el suelo estaba por encima de la capacidad de campo; en estas lluvias se aplicó el Enfoque 2 (13 y 14 de marzo y 28, 29 y 30 de abril).

En la Fig. 15 se muestran los 11 diagramas de la velocidad de infiltración de agua en el suelo de los 12 días de lluvia analizados, la mayoría de los gráficos tienen una duración de menos de $24 \mathrm{~h}$. El suelo muestra un comportamiento muy similar en todas las gráficas, comienza con velocidades más bajas hasta que alcanza un pico máximo y luego disminuye hasta una velocidad estable o básica. La velocidad de infiltración básica se obtuvo considerando los 11 eventos de lluvia, estableciéndose en $1.49 \mathrm{~mm} \cdot \mathrm{h}^{-1}$ con una desviación estándar de $0.36 \mathrm{~mm} \cdot \mathrm{h}^{-1}$. De acuerdo con USDA et al. (1999), esta velocidad se produce cuando el suelo está casi saturado y no disminuye o aumenta a medida que se añade más agua. 
La velocidad de infiltración alcanza una variabilidad del 23\% y podrían deberse a las alteraciones de las trayectorias de flujo, al obligar al agua a pasar por un orificio. Es necesario seguir investigando para reducir al mínimo esta variabilidad, es decir, una serie de orificios situados en el plano horizontal en el fondo del recipiente de suelo podría representar una reducción al mínimo de los errores en la variabilidad de la velocidad de infiltración medida. No obstante, nuestra metodología representa un enfoque para estimar la velocidad de infiltración y podría compararse con otras metodologías.

La variabilidad de la velocidad de infiltración inicial puede deberse a la intensidad de las lluvias y al contenido de humedad inicial (Cui et al., 2019; Liu et al., 2019). Los contenidos de humedad inicial de los siete diagramas de dispersión de la velocidad de infiltración por debajo de la capacidad de campo oscilaron entre 0.23 y $0.30 \mathrm{~cm}^{3} \cdot \mathrm{cm}^{-3}$ y los otros cuatro diagramas de dispersión fueron de 0.30 a $0.33 \mathrm{~cm}^{3} \cdot \mathrm{cm}^{-3}$.
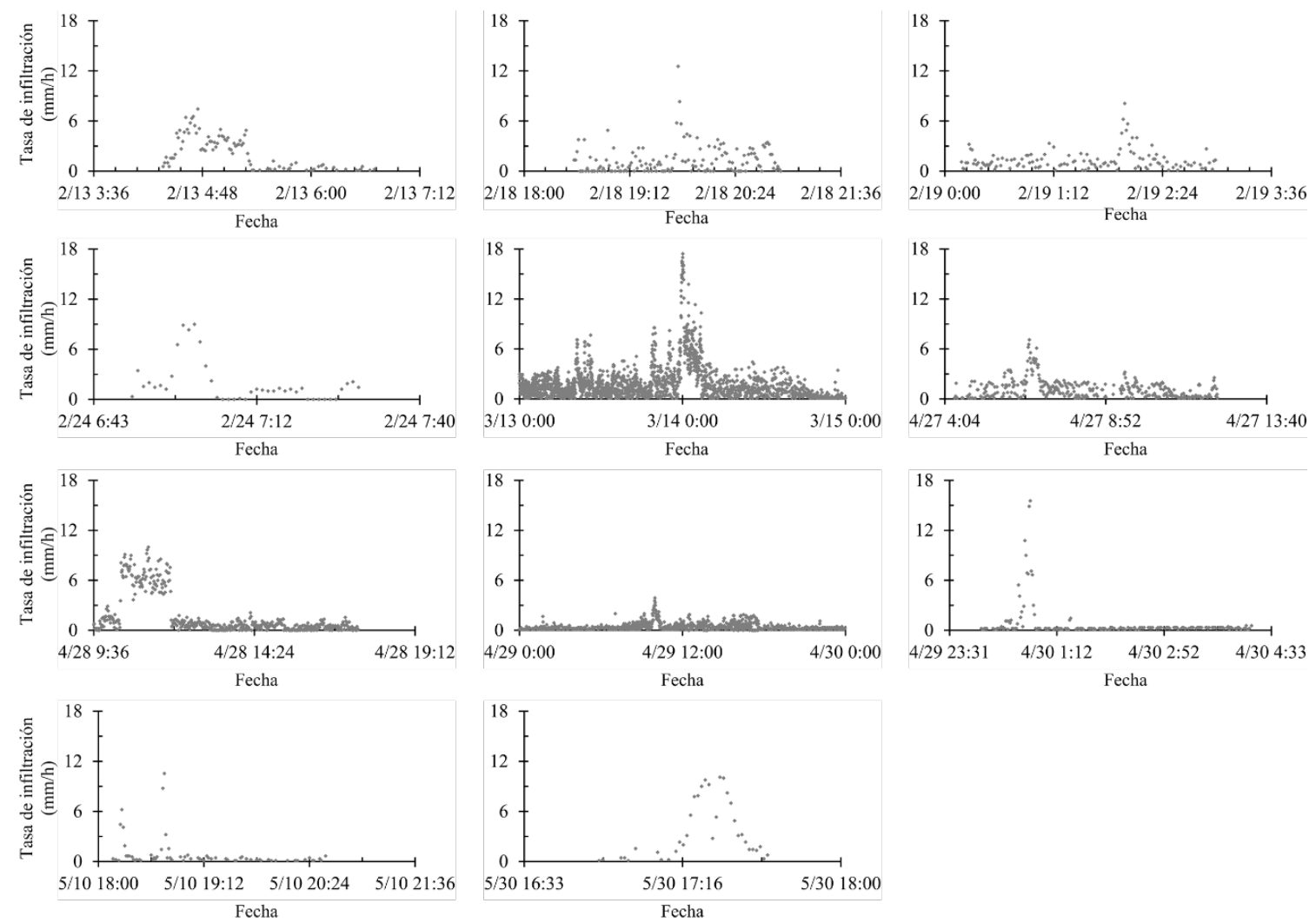

Figura 15. Diagramas de dispersión de la velocidad de infiltración del agua en el suelo. 
La infiltración básica encontrada en ambos enfoques (resumida en la Tabla 4) fue baja comparada con los valores reportados en la literatura; sin embargo, los valores encontrados en esta investigación coinciden con los reportados por Li et al. (2018) para la humectación homogénea, cuyo valor fue obtenido con un contenido inicial de agua en el suelo de $0.034 \mathrm{~m}^{3} \cdot \mathrm{m}^{-3}$, el cual fue secado al aire y colocado en una columna de $50 \mathrm{~cm}$. El valor más bajo obtenido en Li et al. (2018) se obtuvo con un suelo franco limoso ligeramente repelente al agua (esto fue logrado añadiendo octadecilamina al suelo).

La velocidad de infiltración básica obtenida con el lisímetro de pesada fue menor en comparación con los trabajos de investigación de Maldonado (2001) y Ali (2010) considerando el mismo tipo de suelo. Algunos factores que podrían haber causado el bajo valor de la velocidad de infiltración básica son el contenido de materia orgánica, la cubierta vegetal y la raíz del cultivo (Ali, 2010; Liu et al., 2019); sin embargo, se necesita una mayor investigación para corroborarlo.

Los resultados obtenidos con los dos enfoques propuestos son sencillos y representativos de la capacidad actual de infiltración del suelo, donde las raíces han estado creciendo dentro del perfil del suelo, se han añadido fertilizantes y el perfil se ha compactado por la misma fuerza ejercida por el agua de lluvia y el riego. Todo esto no se refleja en los valores que se ven en la Tabla 4.

Tabla 4. Valores de la velocidad de infiltración del agua en un suelo franco limoso.

\begin{tabular}{ccc}
\hline $\begin{array}{c}\text { Velocidad de infiltración } \\
\text { básica, } \mathrm{mm} \cdot \mathrm{h}^{-1}\end{array}$ & \multicolumn{2}{c}{ Método e investigadores } \\
\hline $1.13-1.85$ & Lisímetro de pesada & \\
$10.00-14.00$ & Cilindros concéntricos & (Maldonado, 2001) \\
$2.00-3.00$ & Humectación homogénea & (Li et al., 2018) \\
$0.15-0.30$ & Ligeramente repelente al agua & (Li et al., 2018) \\
$5.00-8.00$ & Cilindros concéntricos & (Ali, 2010) \\
\hline
\end{tabular}




\subsubsection{Calibraciones de modelos}

Los 11 diagramas de infiltración fueron calibrados con los cuatro modelos descritos anteriormente, desde su punto más alto. En la Fig. 16, sólo se presentan los cuatro modelos con mayor duración de las precipitaciones. El modelo que mostró el mejor ajuste fue el de Horton, con un RMSE de $0.89 \pm 0.40$ y R $^{2}$ de $0.65 \pm 0.30$, seguido por Kostiakov con un RMSE de $1.06 \pm 0.40$ y R$^{2}$ de $0.63 \pm 0.23$, Kostiakov Modificado con un RMSE de $1.03 \pm 0.43$ y $\mathrm{R}^{2}$ de $0.62 \pm 0.22$, y finalmente Philip con un RMSE de $1.30 \pm 0.42$ y $\mathrm{R}^{2}$ de $0.48 \pm 0.17$. El único modelo que estableció una velocidad de infiltración inicial más baja fue el de Horton.

Los modelos establecen el valor de la velocidad de infiltración básica en algunas de sus variables de la ecuación. En el caso de Kostiakov no tiene un valor establecido, Kostiakov Modificado estableció la infiltración básica en $0.14 \pm 0.14 \mathrm{~mm} \cdot \mathrm{h}^{-1}$ (en su constante c) (Furman et al., 2006; Strelkoff et al., 2009), Philip en $0.16 \pm 0.11 \mathrm{~mm} \cdot \mathrm{h}^{-1}$ (en A) y Horton en $0.38 \pm 0.34 \mathrm{~mm} \cdot \mathrm{h}^{-1}$ (en $\mathrm{V}_{\mathrm{f}}$ ) (Horton, 1941; Philip, 1957). La calibración de los modelos mostró un comportamiento como el indicado por sus límites, la velocidad de infiltración tiende a valores constantes cercanos a cero cuando el tiempo tiende al infinito (Haverkamp et al., 1988; Philip, 1957).
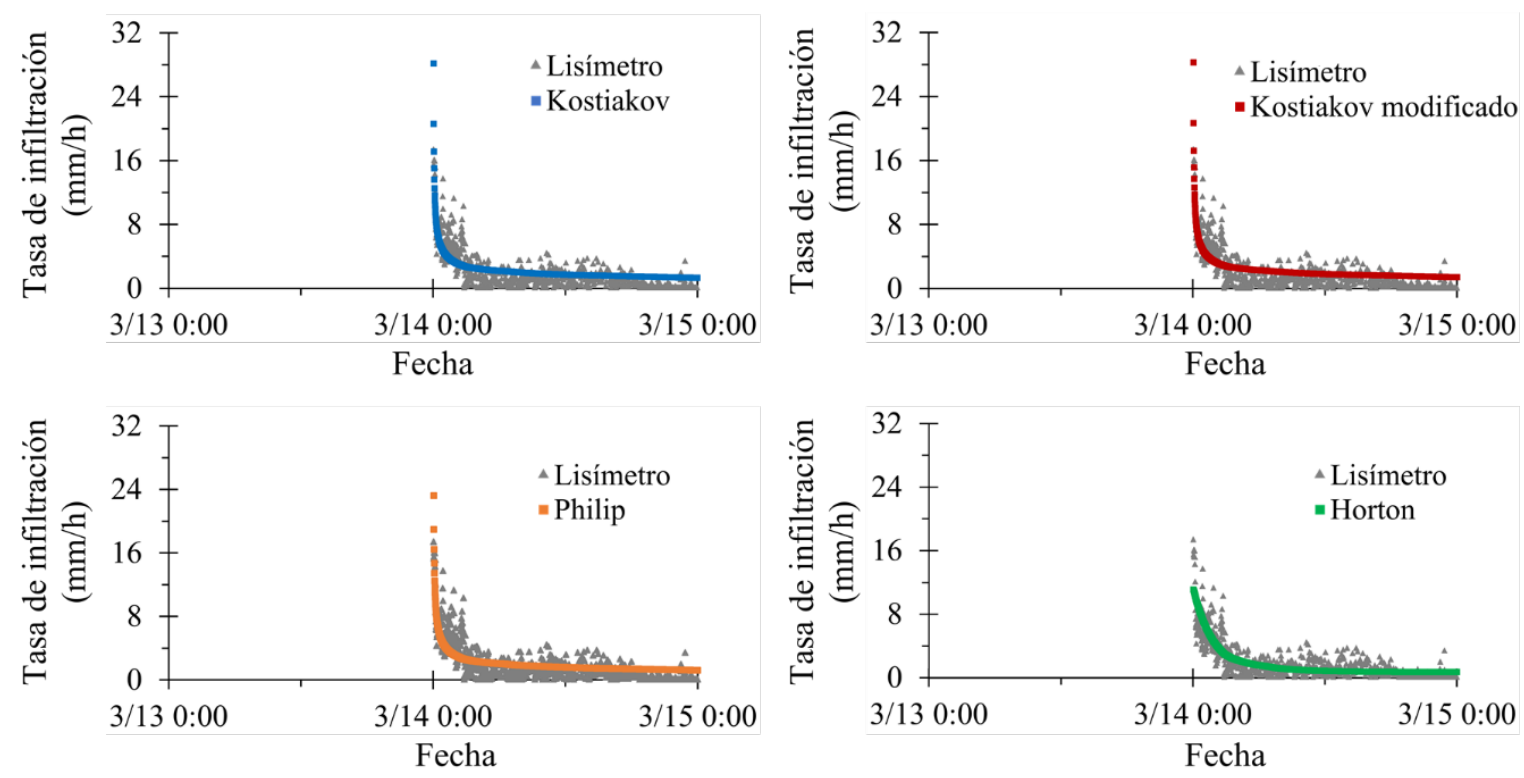

Figura 16. Ajuste de modelos de infiltración a los valores de 1314 de marzo. 
En el trabajo de investigación de Rodríguez Vásquez et al. (2008) y Mirzaee et al. (2014) fue medida la velocidad de infiltración con infiltrómetro de doble anillo para suelos limosos y arcillosos, los valores de sus parámetros obtenidos para los diferentes modelos de infiltración se muestran en la Tabla 5. En el primer trabajo, el contenido de agua se situó entre 24.3 y $31.1 \%$, en el segundo no se mencionó, y nuestro rango fue de 0.23 $0.33 \mathrm{~cm}^{3} \cdot \mathrm{cm}^{-3}$.

Los parámetros de ajuste del modelo de Kostiakov en esta investigación comparados con los de Rodríguez Vásquez et al. (2008), tienen una diferencia del 39\% para el parámetro a, mientras que para el parámetro b es del 20\%. En el modelo de Philip, hay una diferencia de 35\% para el parámetro S y el valor del parámetro A de Rodríguez Vásquez et al. (2008) es 25 veces mayor que el nuestro. Esta diferencia probablemente se debe a que el error de estimación es mayor en los cilindros concéntricos.

Hay una diferencia significativa con respecto al trabajo de Mirzaee et al. (2014) en los cuatro modelos, que varía entre el 68 y el 245\%, porque los valores reportados son de un perfil de suelo con horizontes limo-limoso y arcilloso y la capacidad de infiltración característica de las arcillas es muy baja.

Tabla 5. Valores de los parámetros de los modelos de infiltración para el suelo franco limoso.

\begin{tabular}{ccccc}
\hline Modelo & & $\begin{array}{c}\text { Lisímetro de } \\
\text { pesada }\end{array}$ & $\begin{array}{c}\text { Rodríguez Vásquez } \\
\text { et al. (2008) }\end{array}$ & $\begin{array}{c}\text { Mirzaee } \text { et al. } \\
(2014)\end{array}$ \\
\hline \multirow{2}{*}{ Kostiakov } & $a$ & 47.70 & 66.46 & 1.00 \\
& $b$ & 0.62 & 1.12 & 3.11 \\
Kostiakov & $a$ & 48.42 & & 3.23 \\
Modificado & $b$ & 0.50 & -- & 0.84 \\
& $c$ & 0.29 & & 1.00 \\
Philip & $S$ & 20.83 & 13.60 & 31.30 \\
& $A$ & 0.30 & 7.93 & 1.00 \\
& & & & 58.8 \\
Horton & $V_{O}$ & 14.44 & -- & 34.80 \\
& $V_{f}$ & 0.75 & & 1.00 \\
\hline
\end{tabular}




\subsection{Evapotranspiración del pimiento y coeficiente de cultivo}

\subsubsection{Condiciones meteorológicas}

La temporada del cultivo del 2019 fue del 15 de febrero al 28 de agosto, mientras que la temporada de 2020 fue del 10 de febrero al 22 de agosto, ambas parten desde el día del trasplante. La temperatura media del aire, la humedad relativa media del aire, la velocidad del viento y $\mathrm{ET}_{0}$ para las diferentes temporadas se muestran en la Fig. 17. El comportamiento de las variables climáticas fue muy similar para los dos años. El mes de marzo, lo días finales de abril y los primeros días de junio fueron más cálidos en 2020, mientras que los días finales de junio y principios de julio fueron más cálidos en 2019.
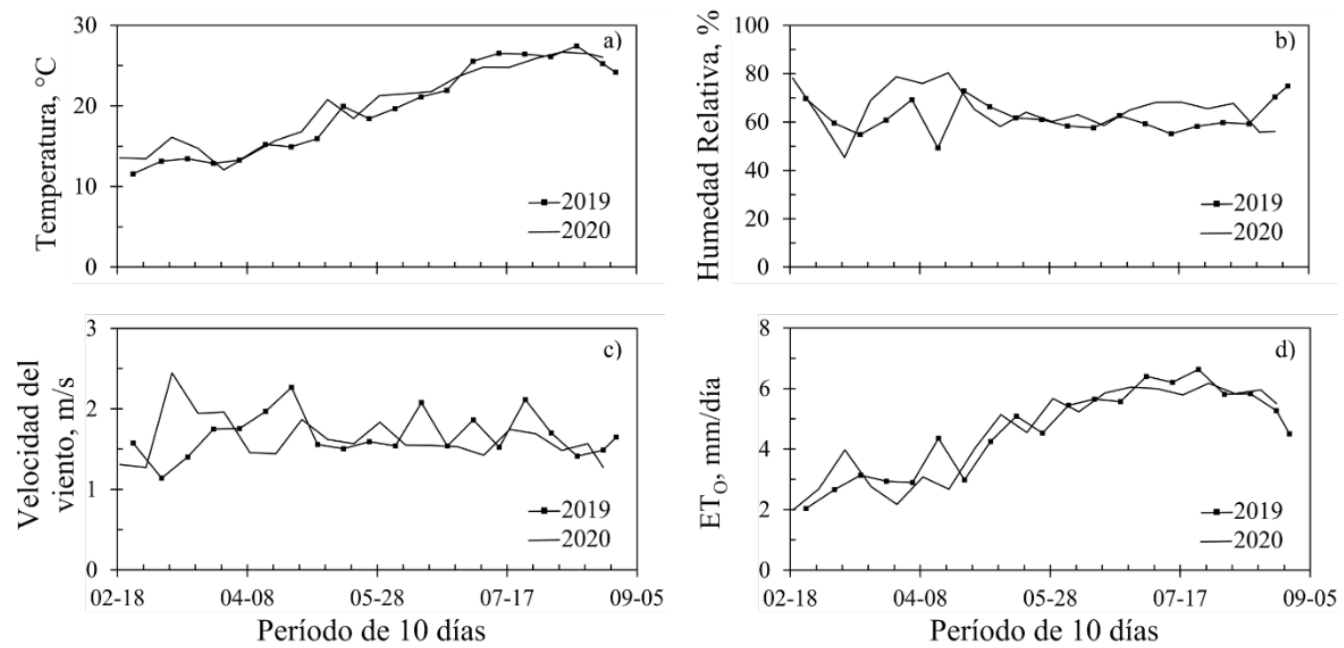

Figura 17. Datos meteorológicos promedio para períodos de 10 días, a) temperatura ambiente, b) humedad relativa, c) velocidad del viento y d) evapotranspiración de referencia $E T_{0}$.

La humedad relativa y velocidad del viento fueron más bajos en 2019 que en 2020 durante la mayor parte de la temporada del cultivo. La humedad relativa fue especialmente baja del 14 de marzo al 18 de abril y del 27 de julio al 10 de agosto en 2020. Los valores medios de humedad relativa registrados para las dos temporadas fueron superiores al $60 \%$, con un promedio mínimo y máximo de $47.3 \%$ y 76.61\%, respectivamente. La velocidad media del viento fue de $1.67 \mathrm{~m} \cdot \mathrm{s}^{-1}$ en $2019 \mathrm{y}$ de $1.65 \mathrm{~m} \cdot \mathrm{s}^{-1}$ en 2020. La ET $\mathrm{ET}_{0}$ aumentó de 
manera similar a la temperatura ambiente, con una $\mathrm{ET}_{0}$ promedio de $4.6 \mathrm{~mm} \cdot$ día $^{-1}$ en 2019 y $4.6 \mathrm{~mm} \cdot$ día $^{-1}$ en 2020.

\subsubsection{Evapotranspiración del cultivo}

La Fig. 18 muestra los valores diarios de ET $_{\mathrm{C}}$ obtenida con el lisímetro, las entradas debidas a la lluvia y riego, y las pérdidas de agua por drenaje. Inicialmente, el riego fue aplicado cada tres días en promedio; sin embargo, de acuerdo al crecimiento del cultivo y el aumento de la $\mathrm{ET}_{\mathrm{C}}$, como efecto de las altas temperaturas del verano, la programación del riego cambio cada dos días, luego disminuyó hasta alcanzar un día entre riegos.
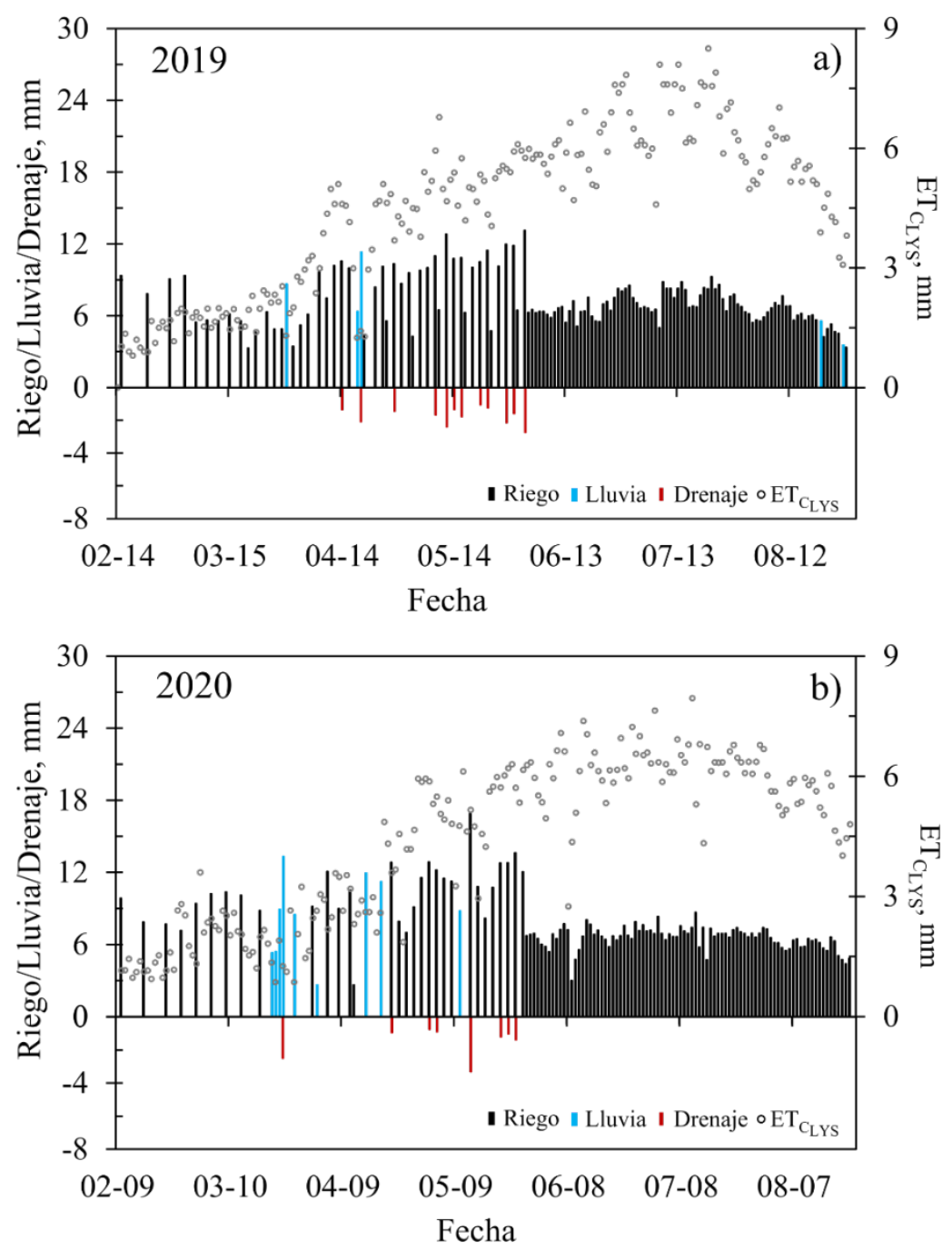

Figura 18. Evapotranspiración diaria del pimiento medido con el lisímetro de pesada (ET $\left.C_{C_{L Y S}}\right)$, riego, lluvia y drenaje, a) 2019 y b) 2020. 
Los intervalos de riego mostraban una tendencia similar en ambas temporadas; el intervalo más amplio entre riegos para la temporada 2019 fue de cinco días y extendido hasta febrero, mientras para la temporada 2020, el intervalo más amplio fue de tres días en promedio y extendido hasta el 20 de abril. En abril, la $\mathrm{ET}_{\mathrm{C}_{\mathrm{LYS}}}$ aumentó, haciendo que el intervalo disminuyera a dos días. En los días de finales de mayo, cuando comenzó el verano, el riego se realizaba diariamente. En este período, la evapotranspiración diaria era superior a 5 mm. Este intervalo comenzó más temprano en la temporada de 2020. La aplicación total del riego fue de 960.3 mm en 2019 y 936.1 mm en 2020. En 2020 hubo más eventos de lluvia, con $40.7 \mathrm{~mm}$ más de lluvia en el doble de eventos, por lo que se aplicó menos riego significando $2.52 \%$ menos de riego aplicado.

La Tabla 6 muestra los valores promedios de riego, lluvia, drenaje y evapotranspiración para ambos años.

Tabla 6. Balance hídrico de temporada en milímetros.

\begin{tabular}{ccccc}
\hline Temporada & Riego & Lluvia & Drenaje & $E T_{C_{L Y S}}$ \\
\hline 2019 & 960.3 & 35.4 & 12.31 & 874.4 \\
2020 & 936.1 & 76.1 & 12.03 & 854.6 \\
\hline
\end{tabular}

Comparando la lámina de agua bruta de riego obtenida con el lisímetro con la obtenida usando la metodología de la FAO-56 fue encontrada una disminución del 1.33\% en 2019 ya que la lámina de agua simulada con la FAO fue de 947.6 mm. En cuanto al año 2020, la lámina simulada fue $928.8 \mathrm{~mm}$, lo que representa una disminución del $0.78 \%$ con respecto a las mediciones del lisímetro. Estas disminuciones fueron pequeñas, la diferencia de las láminas puede significar uno o dos riegos. Los drenajes reportados por el lisímetro alcanzaron un promedio de $12 \mathrm{~mm}$, con 12 y 14 eventos para las temporadas 2019 y 2020, respectivamente.

En la primera temporada, los drenajes no superaron los $4 \mathrm{~mm}$ y fueron causados por los riegos que llegaron a superar los $10 \mathrm{~mm}$ de lámina; por ejemplo, el drenaje causado por las lluvias del 19 al 21 de abril tenía con una lámina de 28.4 mm. En la segunda temporada, 
uno de los drenajes fue causado por la lluvia del 21 al 24 de marzo de con una lámina acumulada de $33 \mathrm{~mm}$ y los otros fueron causados por una lámina de riego mayor a $12 \mathrm{~mm}$. Las principales precipitaciones se produjeron en los meses de marzo y abril; el primer año, hubo cinco días de lluvia con una lámina acumulada de $35.4 \mathrm{~mm}$, mientras que, en el segundo año, hubo lluvias con el doble de días y una lámina acumulada de 76.1 mm.

La $\mathrm{ET}_{\mathrm{C}}$ aumentó cuando la temperatura también lo hizo y cuando el intervalo entre riegos disminuyó, lo que indica la relación que $\mathrm{ET}_{\mathrm{C}}$ tiene con ambas variables. El valor máximo de $\mathrm{ET}_{\mathrm{C}}$ se alcanzó cerca del 16 de julio para ambas estaciones. Cuando la aplicación de riego fue a escala diaria, la $\mathrm{ET}_{\mathrm{C}}$ tuvo valores altos por encima de $5 \mathrm{~mm}$. El comportamiento del $\mathrm{ET}_{\mathrm{C}}$ durante los días de lluvia fue pequeño, y fue mayor el día después del riego o de la lluvia porque la evaporación del suelo húmedo fue más significativa; en este mismo contexto, en los días en que no hubo riego, debido a intervalos más largos, el $\mathrm{ET}_{\mathrm{C}}$ se mantuvo o disminuyó, porque hay menos evaporación de un suelo con superficie seca (Allen et al., 1998). El total de $\mathrm{ET}_{\mathrm{C}}$ para la temporada 2019 fue de $874.4 \mathrm{~mm}$ y el total para 2020, fue de $854.6 \mathrm{~mm}$.

Los valores de $\mathrm{ET}_{\mathrm{C}_{\mathrm{LYS}}}$ medidos con el lisímetro para ambas temporadas se compararon con las $\mathrm{ET}_{\mathrm{O}}$ calculadas con el método $\mathrm{FAO}$ Penman-Monteith y las $\mathrm{ET}_{\mathrm{C}}$ obtenidos con el método establecido por la metodología estándar de la FAO-56 ( $\left(\mathrm{ET}_{\mathrm{C}_{\mathrm{FAO}}}\right)$ (Allen et al., 1998), se presentan en la Fig. 19. El promedio resultante $\mathrm{ET}_{\mathrm{C}_{\mathrm{LYS}}}$ para las etapas inicial, media y final fue de 1.6, 5.8 y $5.4 \mathrm{~mm} \cdot$ día $^{-1}$, respectivamente.

La correlación lineal entre $\mathrm{ET}_{\mathrm{C}_{\mathrm{LYS}}}$ y $\mathrm{ET}_{\mathrm{O}}$ mostró un buen acuerdo (Fig. 19b). El coeficiente de determinación fue de 0.90 y el RMSE fue de $1.04 \mathrm{~mm} \cdot$ día $^{-1}$ para ambas estaciones, lo que indica la dependencia que existe entre las variables. En la comparación de

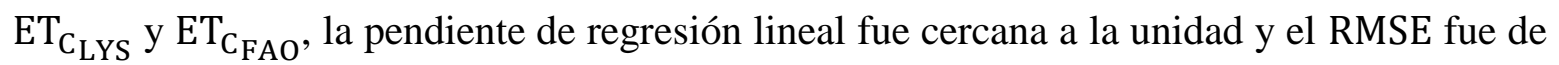
$0.63 \mathrm{~mm} \cdot$ día $^{-1}$. Los valores de $\mathrm{ET}_{\mathrm{C}_{\mathrm{FAO}}}$ en todo el ciclo de cultivo sobrestimaron los $\mathrm{ET}_{\mathrm{C}_{\mathrm{LYS}}}$ en un $37.44 \%$, con un valor medio de $0.4 \mathrm{~mm} \cdot$ día $^{-1}$; esto puede deberse a que los valores $\mathrm{K}_{\mathrm{C}}$ utilizados en la metodología de la FAO-56 eran valores estándar calibrados en condiciones diferentes a las de este experimento. 

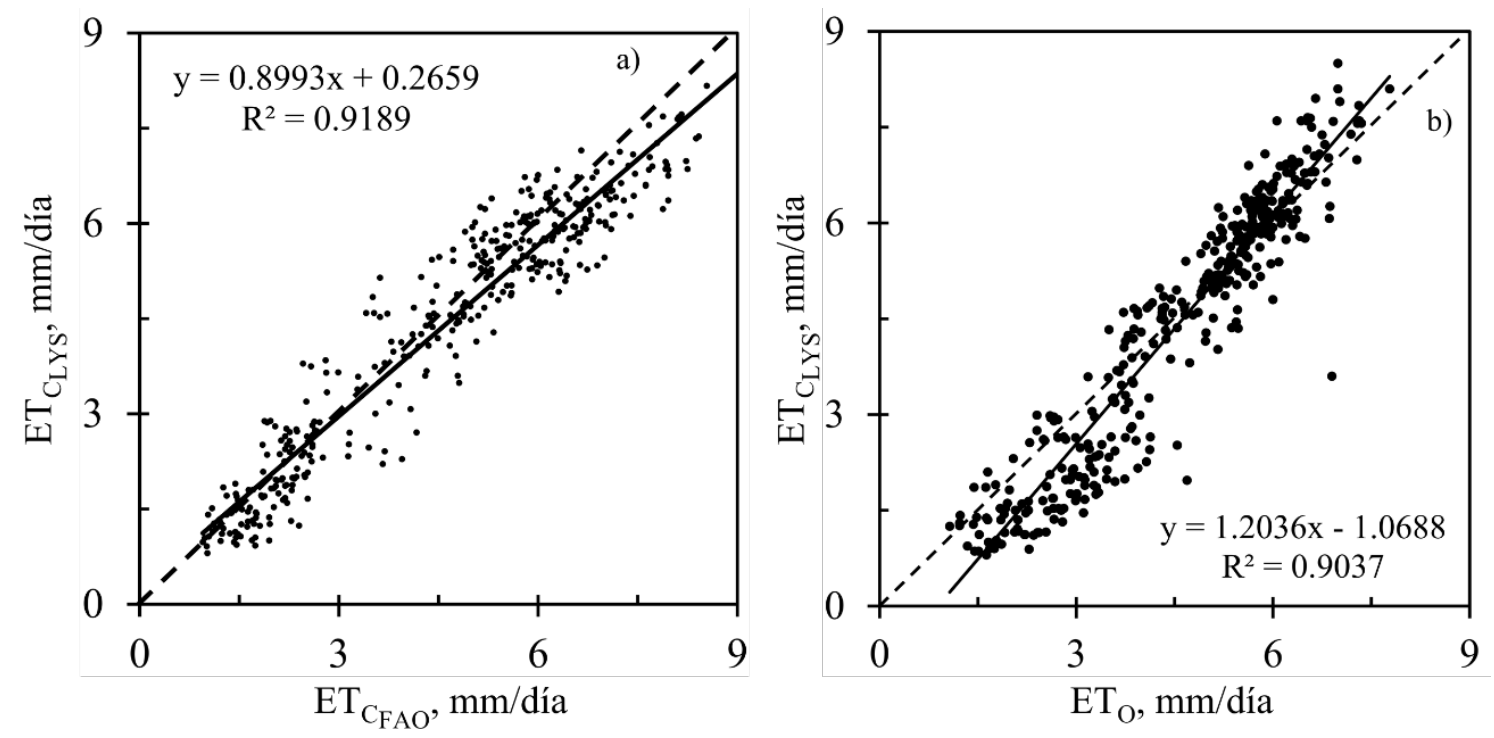

Figura 19. Correlación entre $E T_{C_{L Y S}}$ medido por el lisímetro de pesada y $E T_{C_{F A O}}$ determinado por la metodología de la FAO-56 y entre $E T_{C_{L Y S}}$ y $E T_{O}$.

El MEE fue cercano a $0\left(0.03 \mathrm{~mm} \cdot\right.$ día $^{-1}$ en 2019 y $0.01 \mathrm{~mm} \cdot$ día $^{-1}$ en 2020), lo que indica un bajo sesgo entre $\mathrm{ET}_{\mathrm{C}_{\mathrm{LYS}}} \mathrm{y} \mathrm{ET}_{\mathrm{C}_{\mathrm{FAO}}}$. De manera similar, las otras métricas mostraron el mismo comportamiento. Los valores de IA estuvieron cercanos al uno (0.98 en 2019 y 0.98 en 2020), indicando un buen acuerdo con ambos modelos $\mathrm{ET}_{\mathrm{C}}$. Sin embargo, los valores de $\mathrm{MSE}_{\mathrm{S}}$ fueron de 4.05\% en 2019 y 3.91\% en 2020, lo que indica un alto sesgo, lo que significa que uno de los métodos debe ser mejorado. Los $\mathrm{MSE}_{\mathrm{S}}$ obtenidos por Martínez-Cob, (2008) son muy similares a los resultados de esta investigación, siendo ambos $\mathrm{MSE}_{\mathrm{S}}$ mayores que dos.

\subsubsection{Coeficiente de cultivo}

Los valores de los coeficientes de cultivo estimados a partir de la Ecuación $28\left(\mathrm{~K}_{\mathrm{C}_{\text {lys }}}\right)$ y los valores establecidos en el documento de la FAO-56 para este cultivo $\left(\mathrm{K}_{\mathrm{C}_{\mathrm{FAO}}}\right)$ se muestran en la Fig. 20. El $\mathrm{K}_{\mathrm{C}_{\text {lys inicial }}}$ del lisímetro fue 0.57 , el $\mathrm{K}_{\mathrm{C}_{\text {lys medio }}}$ fue 1.06 , y el $\mathrm{K}_{\mathrm{C}_{\text {lys final }}}$ fue 0.80. Estos datos fueron ajustados a una curva polinómica de tercer grado, con un $\mathrm{R}^{2}$ de 0.73 . En este modelo, se observó un $\mathrm{K}_{\mathrm{C}_{\text {lys }}}$ máximo de 1.11 y un mínimo de 0.48 . 
Al comparar $\mathrm{K}_{\mathrm{C}_{\text {lys }}}$ con $\mathrm{K}_{\mathrm{C}_{\mathrm{FAO}}}$ se obtuvo un $\mathrm{R}^{2}$ de 0.79 y un RMSE de 0.09. Los valores de $\mathrm{K}_{\mathrm{C}_{\mathrm{lys}}}$ oscilaron alrededor de los valores recomendados por Allen et al. (1998).

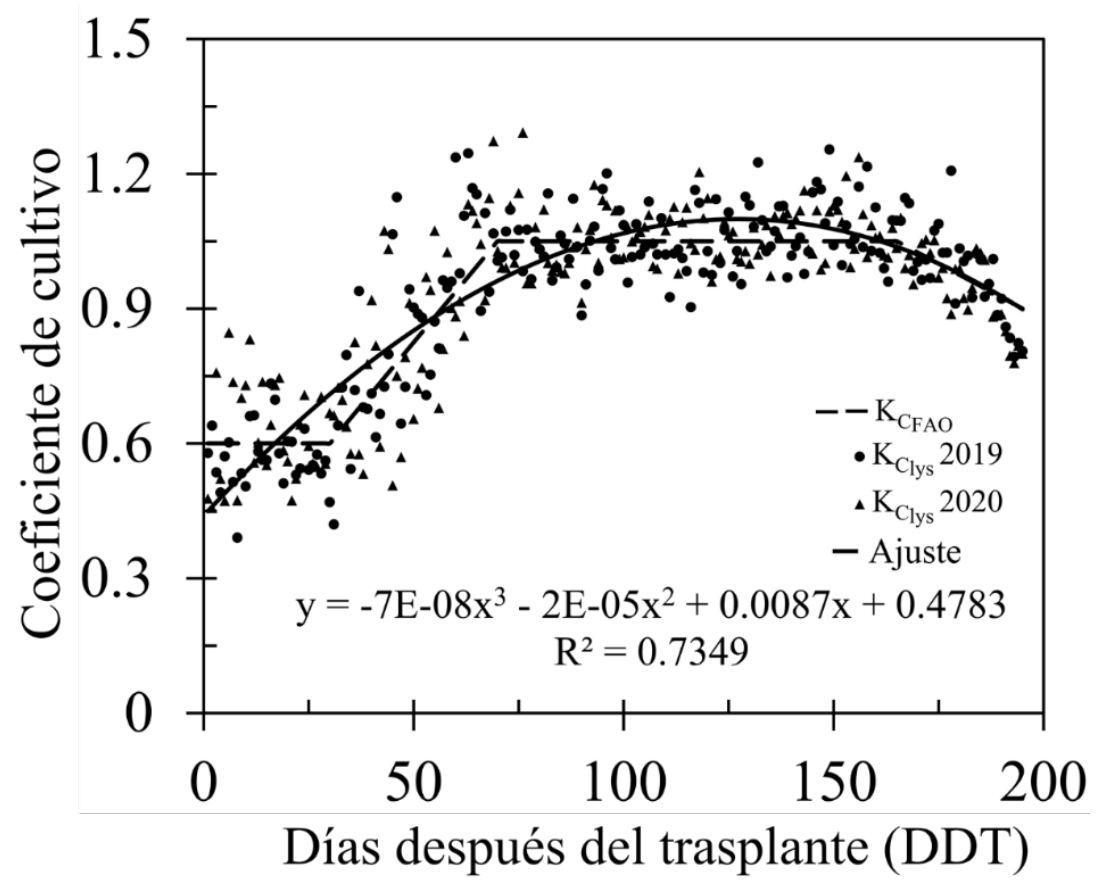

Figura 20. Coeficientes de cultivo del pimiento durante el ciclo de cultivo (valores del lisímetro y la metodología FAO-56).

El salto en $\mathrm{K}_{\mathrm{Clys}_{\text {lys }}}$ entre el 50 y 75 DDT (Fig. 20) puede deberse a los bajos valores de evapotranspiración de referencia reportados en esos días en ambas temporadas, siendo más significativos para la temporada de 2020. Cabe recordar que en la Ecuación 28, el $\mathrm{K}_{\mathrm{C}_{\text {lys }}}$ esta en una función de $\mathrm{ET}_{0}$, por lo que cuanto más bajo sea $\mathrm{ET}_{0}$, más alto será $\mathrm{K}_{\mathrm{C}_{\text {lys }}}$.

Se obtuvo una relación entre $\mathrm{K}_{\mathrm{C}_{\text {lys }}} \mathrm{y}$ las unidades térmicas fraccionales (FTU); la Fig. 21 muestra los dos gráficos resultantes del ajuste polinómico. Como se muestra en la Fig. 21a, el máximo valor de $\mathrm{K}_{\mathrm{C}_{\mathrm{FTU}}}$ fue de 1.15 para el rango de 0.43-0.62 de FTU; el $\mathrm{K}_{\mathrm{C}_{\mathrm{FTU}}}$ máximo de la Fig. 21b fue de 1.15 en el rango de 0.36-0.49. Aunque, el segundo ajuste (Fig. 21b) mostró un $\mathrm{R}^{2}$ ligeramente mejor, alcanzó un máximo de curva durante una etapa temprana del FTU y una disminución más lenta; el final de la curva se elevó y se alejó de los puntos observados. 

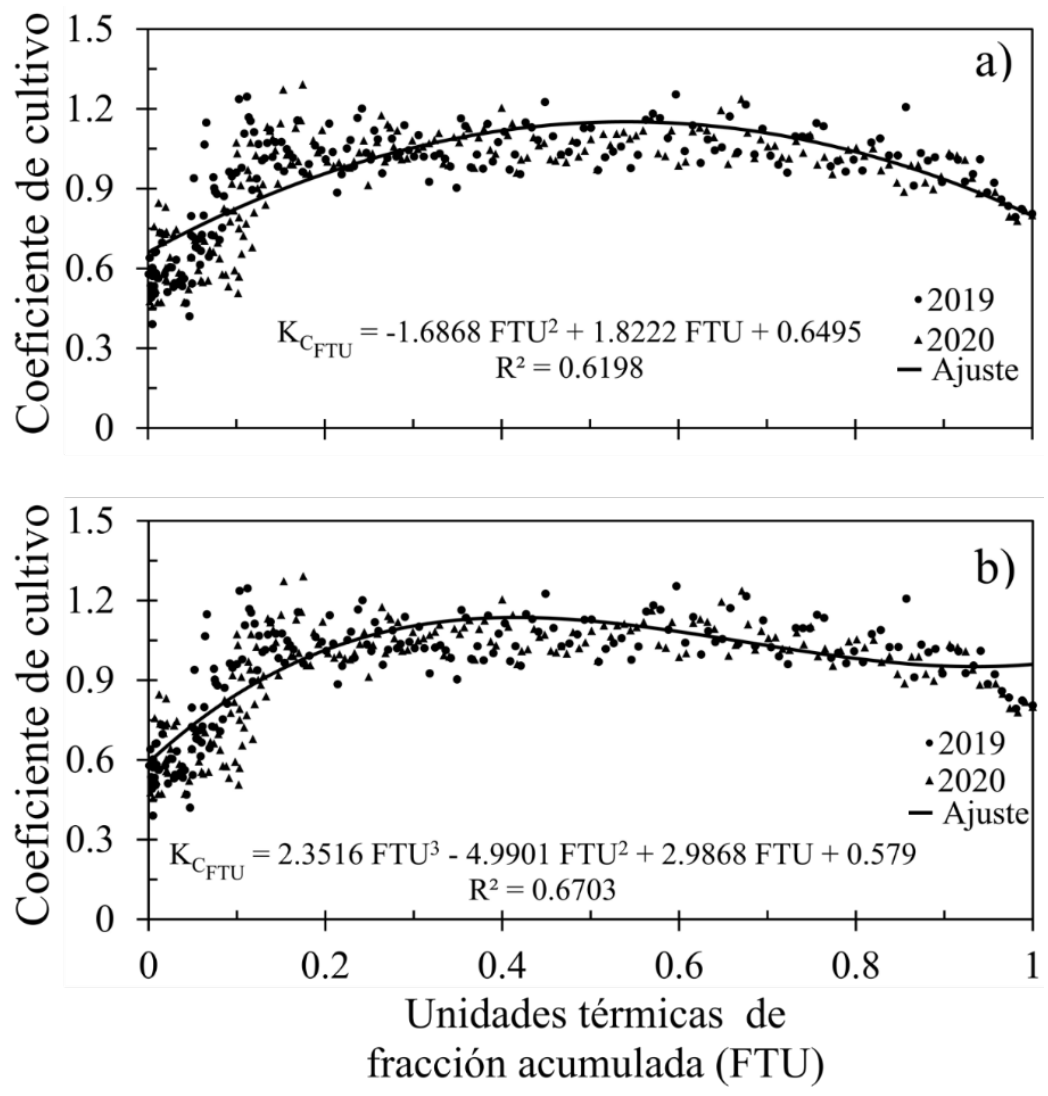

Figura 21. Ajuste polinómico de los valores del coeficiente de cultivo $\left(K_{C}\right)$ obtenidos para 2019 y 2020: a) Ajuste polinómico de segundo orden y b) ajuste polinómico de tercer orden.

La suma promedio de FTU resultante fue de 1881.76, un valor que está dentro del rango propuesto por Vidal (2011). Hubo un sesgo menor entre $K_{C_{F A O}}$ y $K_{C_{F T U}}$, seguido de $\mathrm{K}_{\mathrm{C}_{\mathrm{FAO}}}$ contra $\mathrm{K}_{\mathrm{C}_{\text {lys }}}$ con 0.09 y con 0.48 los valores de $\mathrm{K}_{\mathrm{C}_{\mathrm{FTU}}}$ contra $\mathrm{K}_{\mathrm{C}_{\text {lys }}}$. Estos valores se muestran en la Tabla 7.

La Tabla 7 muestra los valores de $\mathrm{K}_{\mathrm{C}}$ del pimiento estimados en dos trabajos de investigación. El trabajo de Shukla et al. (2013) se realizó en Florida, EE.UU. durante las temporadas de otoño-invierno del 2003 al 2008, con un rango de temperatura de $17-29{ }^{\circ} \mathrm{C}$, una precipitación anual de $1260 \mathrm{~mm} \cdot$ año $^{-1}$, una $\mathrm{ET}_{\mathrm{C}}$ total de $267 \mathrm{~mm}$, y con un nivel freático alto. La cama del cultivo estaba cubierta con mantillo de plástico que cubría el 33\% del área del lisímetro. 
El segundo trabajo de investigación fue realizado por Muniandy et al. (2016) en la zona Peninsular de Malasia, caracterizada por tener un clima cálido y húmedo , durante el mes de agosto de 2013 a mayo de 2014. El rango de temperatura media es de $24-30{ }^{\circ} \mathrm{C}$, una precipitación mensual de 125-270 mm y una humedad relativa (HR) de entre el 63 y el 88\%. Ambas investigaciones utilizaron la misma metodología para determinar la $\mathrm{ET}_{\mathrm{C}} \mathrm{y} \mathrm{K}_{\mathrm{C}}$ que en este estudio.

El rango de temperatura y de la humedad relativa de ambos trabajos estaban en el mismo rango que este trabajo, pero con mayores precipitaciones que las registradas en todo un año y con experimentaciones realizadas en una temporada de siembra diferente en este estudio. Shukla et al. (2013) reportaron valores bimestrales altos de $\mathrm{ET}_{\mathrm{C}}$ para sus temporadas, ya que el riego y las precipitaciones mantuvieron alta la capa freática y aumentaron la humedad del suelo debido al acolchonado. Por lo tanto, los valores $\mathrm{K}_{\mathrm{C}}$ de esa investigación fueron los más altos (Tabla 7), fueron 50.88\%, 14.15\% y 60\% más altos en cada etapa en comparación con los valores reportados con el lisímetro en esta investigación, respectivamente.

En cambio, los valores de Muniandy et al. (2016) en las etapas media y final fueron los más bajos, como se muestra en la Tabla 7. Las condiciones climáticas fueron muy similares a las del trabajo mencionado anteriormente, pero sin la influencia de la capa freática. Estos valores presentaron una reducción del $10.38 \%$ y del $5 \%$ en $\mathrm{K}_{\mathrm{C}_{\text {medio }}} \mathrm{y} \mathrm{K}_{\mathrm{C}_{\text {final }} \text {, }}$, con respecto a los reportados en este trabajo con el lisímetro, respectivamente. Los valores propuestos por Allen et al. (1998) presentaron una menor variabilidad con respecto a los obtenidos con el lisímetro en esta investigación, con un aumento en las etapas inicial y final de $5.26 \%$ y $12.5 \%$, respectivamente, y una disminución en la etapa intermedia de $0.94 \%$. Los valores de la FAO corresponden a climas con una HR cercana al 45\% y velocidades del viento cercanas a $2 \mathrm{~m} \cdot \mathrm{s}^{-1}$; nuestras condiciones satisfacen estas cifras. 
Tabla 7. Valores de $K_{C}$ obtenidos en este estudio y otros trabajos.

\begin{tabular}{|c|c|c|c|c|c|c|c|}
\hline \multirow{2}{*}{$\begin{array}{l}\text { Valores } \\
\text { de } K_{C}\end{array}$} & \multicolumn{3}{|c|}{$\begin{array}{l}\text { Etapa fenológica del } \\
\text { cultivo }\end{array}$} & \multirow[t]{2}{*}{ Localización } & \multirow{2}{*}{$\begin{array}{l}\text { Método de } \\
\text { riego }\end{array}$} & \multirow{2}{*}{$\begin{array}{l}\text { Condiciones } \\
\text { climáticas }\end{array}$} & \multirow{2}{*}{$\begin{array}{c}\text { Ciclo } \\
\text { del } \\
\text { cultivo }\end{array}$} \\
\hline & Inicio & Medio & Final & & & & \\
\hline $\begin{array}{c}\mathrm{K}_{\mathrm{C}_{\text {lys }}} \\
\mathrm{K}_{\mathrm{C}_{\mathrm{FTU}}}{ }^{* *} \\
\mathrm{~K}_{\mathrm{C}_{\mathrm{FTU}}}{ }^{* *}\end{array}$ & $\begin{array}{l}0.57 \\
0.69 \\
0.65\end{array}$ & $\begin{array}{l}1.06 \\
1.05 \\
1.06\end{array}$ & $\begin{array}{l}0.80 \\
0.78 \\
0.93\end{array}$ & $\begin{array}{l}\text { Murcia, } \\
\text { España }\end{array}$ & Goteo & $\begin{array}{c}\text { Temp. } 12-28^{\circ} \mathrm{C} \\
\text { RH } 47 \%-77 \% \\
\text { Lluvia }<100 \\
\mathrm{~mm} \cdot \text { día }^{-1} \\
\mathrm{ET}_{\mathrm{C}} \approx 860 \\
\mathrm{~mm} \cdot \text { temporada }^{-1}\end{array}$ & 195 \\
\hline $\begin{array}{l}\text { Allen et } \\
\text { al. (1998) }\end{array}$ & 0.60 & 1.05 & 0.9 & $\begin{array}{c}\text { Europa y } \\
\text { Mediterráneo }\end{array}$ & -- & $\mathrm{RH} \approx 45 \%$ & 125 \\
\hline $\begin{array}{l}\text { Shukla et } \\
\text { al. (2013) }\end{array}$ & 0.86 & 1.21 & 1.28 & Florida, EUA & $\begin{array}{l}\text { Subsu- } \\
\text { perficial }\end{array}$ & $\begin{array}{c}\text { Temp. } 17-29^{\circ} \mathrm{C} \\
\text { Lluvia } 1260 \\
\mathrm{~mm} \cdot \text { día }^{-1} \\
\mathrm{ET}_{\mathrm{C}} \approx 267 \\
\mathrm{~mm} \cdot \text { temporada }^{-1}\end{array}$ & 100 \\
\hline $\begin{array}{l}\text { Muniandy } \\
\text { et al. } \\
\text { (2016) }\end{array}$ & 0.67 & 0.95 & 0.76 & $\begin{array}{l}\text { Kluang, } \\
\text { Malasia }\end{array}$ & Aspersión & $\begin{array}{c}\text { Temp. } 24-30^{\circ} \mathrm{C} \\
\text { RH } 63 \%-88 \% \\
\text { Lluvia } 125-270 \\
\mathrm{~mm} \cdot \mathrm{mes}^{-1}\end{array}$ & 125 \\
\hline
\end{tabular}

\subsubsection{Rendimiento}

La Tabla 8 muestra un resumen de la producción final alcanzada en cada temporada. El rendimiento de pimiento en ambas temporadas fue superior al reportado por el Ministerio de Medio Ambiente y Medio Rural y Marino (2010) del Gobierno de España en 2019 de 75.66 t·ha-1 para la Región de Murcia. El lisímetro de pesada compacto permitió una mayor precisión en el control del balance hídrico del cultivo, lo que incrementó el rendimiento de este y produjo un importante ahorro de agua en comparación con las prácticas habituales de la Región de Murcia. La producción de agua y los rendimientos económicos fueron similares en ambas temporadas. 
En 2019 se obtuvo un mejor rendimiento, ya que el exceso de humedad del suelo provocado por las lluvias de 2020 generó un aborto de las flores, reduciendo la productividad del pimiento en ese año (López Marín et al. 2017)

Tabla 8. Datos de producción de ambas temporadas.

\begin{tabular}{cccc}
\hline Temporada & $\begin{array}{c}\text { Rendimiento del } \\
\text { pimiento }\left(\mathrm{t} \cdot \mathrm{ha}^{-1}\right)\end{array}$ & $\begin{array}{c}\text { Productividad del } \\
\text { agua }\left(\mathrm{Kg} \cdot \mathrm{m}^{-3}\right)\end{array}$ & $\begin{array}{c}\text { Rendimiento } \\
\text { económico }\left(€ \cdot \mathrm{ha}^{-1}\right)\end{array}$ \\
\hline 2019 & 81.5 & 8.49 & $64,457.55$ \\
2020 & 78.3 & 8.36 & $64,808.91$ \\
\hline
\end{tabular}




\section{Conclusiones}

Los trabajos desarrollados para la elaboración de esta Tesis han permitido obtener las siguientes conclusiones:

a) A partir de los valores de masa de un lisímetro de pesada compacto y de los dos enfoques propuestos fue posible medir el comportamiento de velocidad de infiltración de agua de un suelo franco limoso en condiciones de lluvia inestable. Los resultados mostraron que con los dos enfoques es posible establecer un gráfico de dispersión de la velocidad de infiltración del suelo similar a la típica curva de infiltración, ya sea para tiempos de aplicación cortos y largos en condiciones de campo. El suelo mostró estar sujeto a una velocidad de infiltración variable y más rápida al principio del ingreso del agua, pero alcanza una infiltración básica o estable a medida que el suelo va llenando sus poros.

b) El comportamiento de la humedad en el suelo fue establecido a cada minuto, sin embargo, el primer valor de la humedad tuvo que ser inferido de la densidad aparente del suelo, por lo que es necesario establecer el contenido de humedad inicial en futuras investigaciones. Sin embargo, la metodología usada en este trabajo para la determinación de la humedad del suelo con el lisímetro de pesada queda validada con 
la capacidad de campo del suelo establecida en este trabajo ya que tiene una diferencia menor del 10\% con la encontrada en laboratorio y otros valores de la literatura.

c) Los enfoques de velocidad de infiltración son simples y satisfacen la conservación de la masa y la ecuación de continuidad para flujos incomprensibles. Aunque en este trabajo son utilizan para condiciones de no saturación, pueden utilizarse para condiciones de saturación añadiendo la variable de escorrentía superficial que restaría al flujo entrante al suelo.

d) Estos enfoques permite caracterizar la velocidad de infiltración de un suelo homogéneo y no homogéneo en las profundidades donde la agricultura tiene lugar regularmente; sin embargo, la limitación es que no es posible vigilar el progreso del frente húmedo, por lo que para futuras investigaciones será necesario disponer de mayores instrumentos de medición (es decir, sensores de humedad del suelo) para caracterizar el avance del frente agua en el suelo y las fuerzas que actúan en él (es decir, sensor de potencial de agua del suelo) que ayudarán a verificar que las condiciones dentro del lisímetro sean iguales al suelo circundante.

e) La calibración del modelo infiltración de Horton tuvo mejor ajuste con los datos medidos por el lisímetro, ya que este modelo describe la naturaleza de las curvas típicas de velocidad de infiltración que es la ley exponencial inversa.

f) Los valores $\mathrm{ET}_{\mathrm{C}}$ y $\mathrm{K}_{\mathrm{C}}$ para el cultivo de pimientos fueron calculados usando un lisímetro de pesada compacto y una estación meteorológica automática para dos temporadas en el sureste semiárido de Murcia, España. La $\mathrm{ET}_{\mathrm{C}}$ fue afectada por eventos de riego y lluvia, que aumentaron la evaporación del suelo, más notablemente cuando había un intervalo más corto entre los riegos.

g) Los valores medios de $\mathrm{K}_{\mathrm{C}}$ del pimiento para las etapas inicial, media y final fueron de $0.57,1.06$ y 0.80 , respectivamente. Los valores de $\mathrm{K}_{\mathrm{C}}$ fueron similares a los propuestos por Allen et al. (1998) porque las condiciones climáticas fueron similares en ambos estudios, sin embargo son inferiores en comparación con los valores de 
Shukla et al. (2013), en los que algunas condiciones climáticas fueron similares pero con mayor lluvia y mayor contribución a la capa freática que causaron una mayor evaporación de agua del suelo.

h) Fueron desarrollados modelos de segundo y tercer orden para el $K_{C}$ en función de las unidades térmicas fraccionales, basados en los datos del $\mathrm{K}_{\mathrm{C}}$ inferidos del lisímetro, con coeficientes de determinación superiores a 0.60. Estos modelos servirán para determinar los coeficientes de cultivo a partir de los grados Celsius acumulados de la temperatura en tiempo real, por lo que puede evitarse el uso de los $\mathrm{K}_{\mathrm{C}}$ propuestos por la FAO que son calibrados en condiciones climatológicas diferentes a la zona estudiada y que pueden llevar a crear un sesgo en la programación del riego.

i) Un aumento del rendimiento del pimiento del 7.72\% en 2019 y del 3.49\% en 2020, en comparación con el rendimiento establecido por el Ministerio de Medio Ambiente y Medio Rural y Marino, (2010) del Gobierno Español fue conseguido. Los valores de $\mathrm{K}_{\mathrm{C}_{\text {lys }}} \mathrm{y}$ los modelos de FTU para el pimiento obtenidos en este estudio ayudarán a los agricultores a determinar las necesidades de agua y a mejorar la eficiencia hídrica de los cultivos en lugares semiáridos con condiciones similares a las de este estudio. 



\section{Referencias}

Aboukhaled, A., Alfaro, A., Smith, M., 1982. Lysimeter. FAO (Food Agric. Organ. United Nations) Irrigation.

Adekalu, K.O., Olorunfemi, I.A., Osunbitan, J.A., 2007. Grass mulching effect on infiltration, surface runoff and soil loss of three agricultural soils in Nigeria. Bioresour. Technol. 98, 912-917. https://doi.org/10.1016/j.biortech.2006.02.044

AENOR, 2002. Automatic weather station network. Criteria for the localization of sites and installation of sensor. Adquisition characteristics and sampling. UNE 500520-2002.

Ali, M.H., 2010. Fundamentals of irrigation and on-farm water management. Springer Science \& Business Media.

Allen, R.G., Pereira, L.S., Raes, D., Smith, M., 1998. Crop evapotranspiration-Guidelines for computing crop water requirements-FAO Irrigation and drainage paper 56. Fao, Rome 300, D05109.

Angulo-Jaramillo, R., Bagarello, V., Iovino, M., Lassabatere, L., 2016. Infiltration Measurements for Soil Hydraulic Characterization. Springer. https://doi.org/10.1007/978-3-319-31788-5 
Arriaga, F.J., Kornecki, T.S., Balkcom, K.S., Raper, R.L., 2010. A method for automating data collection from a double-ring infiltrometer under falling head conditions. Soil Use Manag. 26, 61-67. https://doi.org/10.1111/j.1475-2743.2009.00249.x

Assi, A.T., Blake, J., Mohtar, R.H., Braudeau, E., 2019. Soil aggregates structure-based approach for quantifying the field capacity, permanent wilting point and available water capacity. Irrig. Sci. 37, 511-522. https://doi.org/10.1007/s00271-019-00630-w

Babaei, F., Zolfaghari, A.A., Yazdani, M.R., Sadeghipour, A., 2018. Spatial analysis of infiltration in agricultural lands in arid areas of Iran. Catena 170, 25-35. https://doi.org/10.1016/j.catena.2018.05.039

Bagarello, V., Sgroi, A., 2004. Using the single-ring infiltrometer method to detect temporal changes in surface soil field-saturated hydraulic conductivity. Soil Tillage Res. 76, 1324. https://doi.org/10.1016/j.still.2003.08.008

Bello, Z.A., Van Rensburg, L.D., 2017. Development, calibration and testing of a low-cost small lysimeter for monitoring evaporation and transpiration. Irrig. Drain. 66, 263-272. https://doi.org/10.1002/ird.2095

Camarasa Belmonte, A.M., Soriano Garcí, J., López-Garcí, M.J., 2010. The effect of observation timescales on the characterisation of extreme Mediterranean precipitation. Adv. Geosci. 26, 61-64. https://doi.org/10.5194/adgeo-26-61-2010

Cui, Z., Wu, G.L., Huang, Z., Liu, Y., 2019. Fine roots determine soil infiltration potential than soil water content in semi-arid grassland soils. J. Hydrol. 578, 124023. https://doi.org/10.1016/j.jhydrol.2019.124023

de Almeida, W.S., Panachuki, E., de Oliveira, P.T.S., da Silva Menezes, R., Sobrinho, T.A., de Carvalho, D.F., 2018. Effect of soil tillage and vegetal cover on soil water infiltration. Soil Tillage Res. 175, 130-138. https://doi.org/10.1016/j.still.2017.07.009

del Valle Paniagua, E.A., Bueno, G., Oleschko Lutkova, K., 2009. Modelling processes of the Soil Water Regime. Terra Latinoam. 27, 160-168. https://doi.org/10.4028/www.scientific.net/MSF.881.239

Delleur, J.W., 2006. The Handbook of Groundwater Engineering, 2nd ed. 
Duchaufour, P., 1998. Handbook of Pedology. Taylor \& Francis Group.

Elaydi, H., 2017. An Automated Irrigation System for Greenhouses. Am. J. Electr. Electron. Eng. 5, 48-57. https://doi.org/10.12691/ajeee-5-2-3

Escarabajal-Henarejos, D., Molina-Martínez, J.M., Fernández-Pacheco, D.G., CavasMartínez, F., García-Mateos, G., 2015. Digital photography applied to irrigation management of Little Gem lettuce. Agric. Water Manag. 151, 148-157. https://doi.org/10.1016/j.agwat.2014.08.009

Evans, R.G., Sadler, E.J., 2008. Methods and technologies to improve efficiency of water use. Water Resour. Res. 44, 1-15. https://doi.org/10.1029/2007WR006200

Evanylo, G., McGuinn, R., 2000. Agricultural management practices and soil quality.

Fatehnia, M., Paran, S., Kish, S., Tawfiq, K., 2016. Automating double ring infiltrometer with an Arduino microcontroller. Geoderma 262, 133-139. https://doi.org/10.1016/j.geoderma.2015.08.022

Ferguson, B.K., 1994. Stormwater Infiltration. Lewis Publishers.

Fernández-Pacheco, D.G., Escarabajal-Henarejos, D., Ruiz-Canales, A., Conesa, J., MolinaMartínez, J.M., 2014. A digital image-processing-based method for determining the crop coefficient of lettuce crops inthe southeast of Spain. Biosyst. Eng. 117, 23-34. https://doi.org/10.1016/j.biosystemseng.2013.07.014

Fok, Y., 1986. Derivation of Lewis-Kostiakov intake equation. J. Irrig. Drain. Eng. 112, 164171.

Foth, H.D., 1990. Fundamentals of soil science, 8th ed. John Wiley \& Son, Michigan State University.

Fuentes Ruíz, C., Quiñones Pedroza, H., Rendón Pimentel, L., Hernández Saucedo, F.R., Sánchez Bravo, R., Magaña Sosa, G., Peña Peña, E., Enciso Medina, J., Martínez Sainos, F., Carrillo García, M., Herrera Ponce, J.C., Angeles Montiel, V., Castillo González, J., Martínez Austria, P., Replogle, J.D., Clemmens, A.J., de León Mojarro, B., Ruíz Carmona, V.M., Iñiguez Cobarrubias, M., Angeles Hernández, J.M., Sánchez 
Astello, M., Arteaga Tovar, R.E., 1997. Manual para diseño de zonas de riego pequeñas, Departamen. ed. Instituto Mexicano de Tecnología del Agua, México.

Furman, A., Warrick, A.W., Zerihun, S.C.A., 2006. Modified Kostiakov Infiltration Function: Accounting for Initial and Boundary Conditions. J. Irrig. Drain. Eng. 132, 587-596. https://doi.org/10.1061/(ASCE)0733-9437(2006)132

Gallardo, M., Thompson, B., Fernández, M.D., 2013. Water requirements and irrigation management in Mediterranean greenhouses: the case of the southeast coast of Spain, in: Food and Agriculture Organization of the United Nations (Ed.), Good Agricultural Practices for Greenhouse Vegetable Crops. Principle for Mediterranean Climate Areas. Rome, pp. 109-136.

García-Tejero, I.F., Durán-Zuazo, V.H., Muriel-Fernández, J.L., Rodríguez-Pleguezuelo, C.R., 2011. Water and Sustainable Agriculture. Springer 1-16. https://doi.org/10.1007/978-94-007-2091-6_1

Gebler, S., Hendricks Franssen, H.J., Pütz, T., Post, H., Schmidt, M., Vereecken, H., 2015. Actual evapotranspiration and precipitation measured by lysimeters: A comparison with eddy covariance and tipping bucket. Hydrol. Earth Syst. Sci. 19, 2145-2161. https://doi.org/10.5194/hess-19-2145-2015

Haghiabi, A.H., Abedi-Koupai, J., Heidarpour, M., Mohammadzadeh-Habili, J., 2011. A new method for estimating the parameters of Kostikov and modified Kostiakov infiltration equations. World Appl. Sci. J. 15, 129-135.

Hannes, M., Wollschläger, U., Schrader, F., Durner, W., Gebler, S., Pütz, T., Fank, J., von Unold, G., Vogel, H.-J., 2015. High-resolution estimation of the water balance components from high-precision lysimeters. Hydrol. Earth Syst. Sci. Discuss. 12, 569608. https://doi.org/10.5194/hessd-12-569-2015

Hartley, D.M., 1992. Interpretation of Kostiakov infiltration parameters for borders. J. Irrig. Drain. Eng. 118, 156-165.

Haselow, L., Meissner, R., Rupp, H., Miegel, K., 2019. Evaluation of precipitation measurements methods under field conditions during a summer season: A comparison 
of the standard rain gauge with a weighable lysimeter and a piezoelectric precipitation sensor. J. Hydrol. 575, 537-543. https://doi.org/10.1016/j.jhydrol.2019.05.065

Haverkamp, R., Kutilek, M., Parlange, J.Y., Rendon, L., M., K., 1988. Infiltration under ponded conditions: 2.Infiltration equations tested for parameters time-dependece and predictive use1. Soil Sci. 145, 317-329.

Herbrich, M., Gerke, H.H., 2016. Autocorrelation analysis of high resolution weighing lysimeter time series as a basis for determination of precipitation. J. Plant Nutr. Soil Sci. 179, 784-798. https://doi.org/10.1002/jpln.201600169

Herrada, M.A., Gutiérrez-Martin, A., Montanero, J.M., 2014. Modeling infiltration rates in a saturated/unsaturated soil under the free draining condition. J. Hydrol. 515, 10-15. https://doi.org/10.1016/j.jhydrol.2014.04.026

Hoffman, M., Schwartengräber, R., Wessolek, G., Peters, A., 2016. Comparison of simple rain gauge measurements with precision lysimeter data. Atmos. Res. 174-175, 120123.

Horton, R.E., 1941. Approach toward a physical interpretation of infiltration capacity. Soil Sci. Soc. Am. J. 5, 339-417.

Jensen, M.E., 2007. Beyond irrigation efficiency. Irrig. Sci. 25, 233-245. https://doi.org/10.1007/s00271-007-0060-5

Katyara, S., Shah, M.A., Zardari, S., Chowdhry, B.S., Kumar, W., 2017. WSN Based Smart Control and Remote Field Monitoring of Pakistan's Irrigation System Using SCADA Applications. Wirel. Pers. Commun. 95, 491-504. https://doi.org/10.1007/s11277-0163905-5

Kirkham, M.B., 2010. Principles of Soil and Plant Water Relations. Academic Press.

Koorevaar, P., Menelik, G., Dirksen, C., 1999. Elements of soil physics., 5th ed, Elements of soil physics. Elsevier Science B.V. https://doi.org/10.1097/00010694-19851000000012

Kullberg, E.G., DeJonge, K.C., Chávez, J.L., 2017. Evaluation of thermal remote sensing 
indices to estimate crop evapotranspiration coefficients. Agric. Water Manag. 179, 6473. https://doi.org/10.1016/j.agwat.2016.07.007

Lewis, M.R., 1937. The rate of infiltration of water in irrigation-practice. Trans. Am. Geophys. Union 18, 361-368.

Li, Y., Ren, X., Hill, R., Malone, R., Zhao, Y., 2018. Characteristics of Water Infiltration in $\begin{array}{lllll}\text { Layered } \quad \text { Water-Repellent } \quad \text { Soils. } & \text { Pedosphere }\end{array}$ https://doi.org/10.1016/S1002-0160(17)60414-4

Liu, Y., Cui, Z., Huang, Z., López-Vicente, M., Wu, G.L., 2019. Influence of soil moisture and plant roots on the soil infiltration capacity at different stages in arid grasslands of China. Catena 182, 104147. https://doi.org/10.1016/j.catena.2019.104147

López Marín, J., Angosto, J.L., González, A., 2017. El cultivo de pimientos en invernadero y al aire libre - El caso del Campo de Cartagena. Bibl. Hortic. 68.

Mahmood, S., Latif, M., 2005. A simple procedure for simulating surge infiltration using first-surge infiltrometer data. Irrig. Drain. 54, 407-416. https://doi.org/10.1002/ird.192

Maldonado, T., 2001. Manual de Riego Parcelario [WWW Document]. Santiago Chile FAO. URL

http://www.fao.org/tempref/GI/Reserved/FTP_FaoRlc/old/prior/recnat/pdf/MR_cap1. PDF

Martínez-Cob, A., 2008. Use of thermal units to estimate corn crop coefficients under semiarid climatic conditions. Irrig. Sci. 26, 335-345. https://doi.org/10.1007/s00271007-0097-5

Masarik, K.C., Norman, J.M., Brye, K.R., Baker, J.M., 2004. Improvements to measuring water flux in the vadose zone. J. Environ. Qual. 33, 1152-1158.

Mateos, L., González-Dugo, M.P., Testi, L., Villalobos, F.J., 2013. Monitoring evapotranspiration of irrigated crops using crop coefficients derived from time series of satellite images. I. Method validation. Agric. Water Manag. 125, 81-91. https://doi.org/10.1016/j.agwat.2012.11.005 
Mattar, M.A., Alazba, A.A., Zin El-Abedin, T.K., 2015. Forecasting furrow irrigation infiltration using artificial neural networks. Agric. Water Manag. 148, 63-71. https://doi.org/10.1016/j.agwat.2014.09.015

Meissner, R., Seeger, J., Rupp, H., Seyfarth, M., Borg, H., 2007. Measurement of dew, fog, and rime with a high-precision gravitation lysimeter. J. Plant Nutr. Soil Sci. 170, 335344. https://doi.org/10.1002/jpln.200625002

Ministerio de Agricultura Pesca y Alimentación, 2020. Guía práctica de la fertilización racional de los cultivos en España [WWW Document]. URL https://www.mapa.gob.es/es/agricultura/publicaciones/Publicaciones-fertilizantes.aspx

Ministerio de Medio Ambiente y Medio Rural y Marino, 2010. Anuario de Estadistica 2010. Madrid, España.

Miranda, F.R., Gondim, R.S., Costa, C.A.G., 2006. Evapotranspiration and crop coefficients for tabasco pepper (Capsicum frutescens L.). Agric. Water Manag. 82, 237-246. https://doi.org/10.1016/j.agwat.2005.07.024

Mirzaee, S., Zolfaghari, A.A., Gorji, M., Dyck, M., Ghorbani Dashtaki, S., 2014. Evaluation of infiltration models with different numbers of fitting parameters in different soil texture classes. Arch. Agron. Soil Sci. 60, 681-693. https://doi.org/10.1080/03650340.2013.823477

Moreno, F., Martin, J., Arrue, J.L., 1978. Relacion entre Textura y Retención de Humedad para el Margen de pF 4,2-6,0, en Suelos de Andalucia Occidental. An. Edafol. y Agrobiol. 335-344.

Muniandy, J.M., Yusop, Z., Askari, M., 2016. Evaluation of reference evapotranspiration models and determination of crop coefficient for Momordica charantia and Capsicum annuum. Agric. Water Manag. 169, 77-89. https://doi.org/10.1016/j.agwat.2016.02.019

Navarro-Hellín, H., Martínez-del-Rincon, J., Domingo-Miguel, R., Soto-Valles, F., TorresSánchez, R., 2016. A decision support system for managing irrigation in agriculture. Comput. Electron. Agric. 124, 121-131. https://doi.org/10.1016/j.compag.2016.04.003

Nicolás-Cuevas, J.A., Parras-Burgos, D., Soler-Méndez, M., Ruiz-Canales, A., Molina- 
Martínez, J.M., 2020. Removable weighing lysimeter for use in horticultural crops. Appl. Sci. 10. https://doi.org/10.3390/app10144865

Ooi, S.K., Cooley, N., Mareels, I., Dunn, G., Dassanayake, K., Saleem, K., 2010. Automation of on-farm irrigation: Horticultural case study. IFAC Proc. Vol. 43, 256-261. https://doi.org/10.3182/20101206-3-jp-3009.00045

Parra, L., Botella-Campos, M., Puerto, H., Roig-Merino, B., Lloret, J., 2020. Evaluating Irrigation Efficiency with Performance Indicators: A Case Study of Citrus in the East of Spain. Agronomy 10, 1359. https://doi.org/10.3390/agronomy10091359

Pereira, L.S., de Juan Valero, J.A., Picornell Buendía, M.R., Tarjuelo Martín-Benito, J.M., 2010. El Riego y sus Tecnologías. CREA-UCLM, Albacete, España.

Peters, A., Nehls, T., Schonsky, H., Wessolek, G., 2014. Separating precipitation and evapotranspiration from noise - A new filter routine for high-resolution lysimeter data. Hydrol. Earth Syst. Sci. 18, 1189-1198. https://doi.org/10.5194/hess-18-1189-2014

Philip, J.R., 1957. The Theory of Infiltration: 4. Sorptivity and Algebraic Infiltration Equation. Soil Sci. 84, 257-264.

Plaster, E.J., 2013. Soil Science \& Management, 6th ed. CENGAGE Learning.

Playán, E., Mateos, L., 2006. Modernization and optimization of irrigation systems to increase water productivity. Agric. Water Manag. 80, 100-116. https://doi.org/10.1016/j.agwat.2005.07.007

Porta-Casanellas, J., Lopez-Acevedo, R.M., 2005. Agenda de campo de suelos. Información de suelos para la agricultura y el medio ambiente. Ediciones Mundiprensa.

Richards, L.A., 1931. Capillary conduction of liquids through porous mediums. J. Appl. Phys. 1, 318-333. https://doi.org/10.1063/1.1745010

Rodríguez Vásquez, A.F., Aristizábal Castillo, A.M., Camacho Tamayo, J.H., 2008. Variabilidad espacial de los modelos de infiltración de Philip y Kostiakov en un suelo Ándico. Eng. Agríc., Jaboticabal 28, 64-75. https://doi.org/10.12816/0013114

Ruiz-Peñalver, L., Vera-Repullo, J.A., Jiménez-Buendía, M., Guzmán, I., Molina-Martínez, 
J.M., 2015. Development of an innovative low cost weighing lysimeter for potted plants: Application in lysimetric stations. Agric. Water Manag. 151, 103-113. https://doi.org/10.1016/j.agwat.2014.09.020

Sanchéz-Bravo, P., Chambers V, E., Noguera-Artiaga, L., Sendra, E., Chambers IV, E., Carbonell-Barrachina, Á.A., 2020. How Consumers Perceive Water Sustainability ( HydroSOStainable ) in Food Products and How to Identify It by a Logo. Agronomy 10, $1-13$.

Sandhu, O.S., Gupta, R.K., Thind, H.S., Jat, M.L., Sidhu, H.S., Yadvinder-Singh, 2019. Drip irrigation and nitrogen management for improving crop yields, nitrogen use efficiency and water productivity of maize-wheat system on permanent beds in north-west India. Agric. Water Manag. 219, 19-26. https://doi.org/10.1016/j.agwat.2019.03.040

Schrader, F., Durner, W., Fank, J., Gebler, S., Pütz, T., Hannes, M., Wollschläger, U., 2013. Estimating Precipitation and Actual Evapotranspiration from Precision Lysimeter Measurements. Procedia Environ. Sci. 19, 543-552. https://doi.org/10.1016/j.proenv.2013.06.061

Seiler, K.-P., Gat, J.R., 2007. Groundwater Recharge form Run-off, Infiltration and Percolation. Springer.

Shukla, S., Jaber, F.H., Goswami, D., Srivastava, S., 2013. Evapotranspiration losses for pepper under plastic mulch and shallow water table conditions. Irrig. Sci. 31, 523-536. https://doi.org/10.1007/s00271-012-0327-3

Smerdon, E.T., Blair, W., Reddell, D.L., 1988. Infiltration from irrigation advance data 114, $4-17$.

Smith, R.E., Smettem, K.R.J., Broadbridge, P., Woolhiser, D.A., 2002. Infiltration Theory for Hydrologic Applications, Water Reso. ed. American Geophysical Union, Washington, DC.

Staff, S. survey, 1951. Soil Survey Manual. U.S. Dept. Agric. Handb. 34, 145. https://doi.org/10.2307/1233734

Strelkoff, T.S., Clemmens, A.J., Bautista, E., 2009. Field properties in surface irrigation 
management and design. J. Irrig. Drain. Eng. 135, 525-536. https://doi.org/10.1061/(ASCE)IR.1943-4774.0000119

Tarjuelo Martín-Benito, J.M., 1991. El riego por aspersión: diseño y funcionamiento, 3rd ed. Universidad de Castilla La Mancha.

Teófilo-Salvador, E., Morales-Reyes, G.P., 2018. Proposal of the physical model of the multifunctional redesigned double ring infiltrometer (ICCRM). Tecnol. y Ciencias del Agua 9, 103-131. https://doi.org/10.24850/j-tyca-2018-06-05

USDA, U.S.D. of A., NRCS, N.R.C.S., ARS, A.R.S., Institute Soil Quality, 1999. Soil Qualit. Test Kit Guide.

USGS, 1993. The distribution of water on, in, and above the Earth. [WWW Document]. United States Geol. Surv. URL https://www.usgs.gov/media/images/distribution-waterand-above-earth-0 (accessed 12.10.20).

Verma, S.P., 2016. Análisis Estadístico de Datos Composicionales. Universidad Autónoma de México, Ciudad de México, México.

Vico, G., Porporato, A., 2010. Traditional and microirrigation with stochastic soil moisture. Water Resour. Res. 46, 1-11. https://doi.org/10.1029/2009WR008130

Vidal, J.L., 2011. Efectos del factor térmico en el desarrollo del crecimiento inicial de pimiento (Capsicum annum L.) cultivado en campo. Universidad Nacional de Tucumán, Tucumán, Argentina.

Villalobos, F.., Mateos, L., Orgaz, F., Fereres, E., 2002. Fitotecnia bases y tecnologías de la producción agrícola. Mundi-Pren, Madrid, España.

Wackerly, D.D., Mendenhall, W., Schaeaffer, R.L., 2010. Mathematical statics with applications, 7th ed. Learning Cengage.

Wang, K., Yang, X., Liu, X., Liu, C., 2017. A simple analytical infiltration model for short$\begin{array}{llll}\text { duration } & \text { rainfall. } & \text { J. } & \text { Hydrol. }\end{array}$ https://doi.org/10.1016/j.jhydrol.2017.09.049

Wang, N., Chu, X., 2020. Revised Horton model for event and continuous simulations of 
infiltration. J. Hydrol. 589, 125215. https://doi.org/10.1016/j.jhydrol.2020.125215

Willmott, C.J., Ackleson, S.G., Davis, R.E., Feddema, J.J., Klink, K.M., Legates, D.R., O’Donnell, J., Rowe, C.M., 1985. Statistics for the evaluation and comparison of models. J. Geophys. Res. 90, 8995. https://doi.org/10.1029/jc090ic05p08995

Yang, M., Zhang, Y., Pan, X., 2020. Improving the Horton infiltration equation by considering soil moisture variation. J. Hydrol. 586, 124864. https://doi.org/10.1016/j.jhydrol.2020.124864

Yuan, J., Feng, W., Jang, X., Wang, J., 2019. Saline-alkali migration in soda saline soil based on sub-soiling technology. Desalin. Water Treat. 149, 352-362. https://doi.org/10.5004/dwt.2019.23856 



\section{Producción científica}

\subsection{Artículos de difusión}

\subsubsection{Revista Agrícola Vergel}

1. Soler-Méndez, M., Ávila-Dávila, L., González-Trinidad, J., Ruiz-Canales, A., Molina-Martínez, J.M. 2019. Control de precisión del balance hídrico en tiempo real en cultivos hortícolas. Agrícola Vergel, Revista de Fruticultura, Horticultura, Vid, Arroz. 418,104-106.

2. Ávila-Dávila, L., Soler-Méndez, M., Bautista-Capetillo, C.F., Ruiz-Canales, A., Molina-Martínez, J.M. 2019. Digitalización de cultivos hortícolas para riego eficiente en tiempo real. Agrícola Vergel, Revista de Fruticultura, Horticultura, Vid, Arroz. 14.

\subsection{Publicaciones en congresos}

\subsubsection{Jornadas Doctorales}

Celebrado en la Universidad de Murcia en Murcia, España del 29 al 31 de mayo del 2019. 
1. Ávila-Dávila L., Soler-Méndez M., Bautista-Capetillo C.F., González-Trinidad J., y Molina-Martínez J.M. Modelos de infiltración empleados en la dinámica del agua en suelos agrícolas

\subsubsection{Congreso Ibérico de Agroingeniería 2019}

Celebrado en la Escuela Politécnica Superior (Universidad Zaragoza), Huesca, España del 3 al 6 de septiembre del 2019.

1. Ávila-Dávila, L., Soler-Méndez, M., Madrona-Sánchez, P., Ruiz-Canales, A., Bautista-Capetillo, C.F., González-Trinidad, J., y Molina-Martínez, J.M. Análisis de la velocidad de infiltración del agua en suelos saturados mediante lisímetros de pesada. Proceedings of the 10th Iberian Agroengineering Congress. 2019, 642-648. DOI: 10.26754/c_agroing.2019.com.3370

2. Ávila-Dávila, L., Soler-Méndez, M., Ruiz-Peñalver, L., Bautista-Capetillo, C.F., y Molina-Martínez, J.M. Empleo de sumadores electrónicos en lisímetros de pesada de varías de células de carga. Proceedings of the 10th Iberian Agroengineering Congress. 2019, 953-958. DOI: 10.26754/c_agroing.2019.com.3392

3. Soler-Méndez, M., Madrona-Sánchez, P., Ávila-Dávila, L., Ruiz-Canales, A., y Molina-Martínez, J.M. Análisis del consumo de agua de Hordeum vulgare L. mediante un equipo de lisimetría de pesada. Proceedings of the 10th Iberian Agroengineering Congress. 2019, 906-910. DOI: 10.26754/c_agroing.2019.com.3377

4. Soler-Méndez, M., Molina-Martínez, J.M., Ávila-Dávila, L., y Ruiz-Peñalver, L. Automatización de pequeños jardines con Sonoff 4CH Pro R2 y EWelink. Proceedings of the 10th Iberian Agroengineering Congress. 2019, 911-916. DOI: 10.26754/c_agroing.2019.com.3376

5. Nicolás-Cuevas, J.A., Parras-Burgos, D., Ávila-Dávila, L., Soler-Méndez, M., Ruiz-Peñalver, L., y Molina-Martínez, J.M. Implementación de depósitos de muestreo en lisímetros de pesada y sus necesidades. Proceedings of the 10th Iberian 
Agroengineering Congress. 2019, 795-799. DOI:

10.26754/c_agroing.2019.com.3449

6. Nicolás-Cuevas, J.A., Parras-Burgos, D., Ávila-Dávila, L., Soler-Méndez, M., González-Trinidad, J., y Molina-Martínez, J.M. Evolución de prototipos de depósitos de drenaje para lisímetros de pesada. Proceedings of the 10th Iberian $\begin{array}{llll}\text { Agroengineering } \quad \text { 2019, } & \text { Congress. }\end{array}$ 10.26754/c_agroing.2019.com.3450

\subsubsection{Symposium Ibérico de Ingeniería Hortícola 2020}

Celebrado en la Escuela Superior Agraria del Instituto Politécnico de Viana Do Castelo del 4 al 6 marzo, en Refóios do Lima, Ponte de Lima (Portugal). Organizado por la Asociación Portuguesa de Horticultura (APH) junto con la sección de Ingeniería Hortícola de la Sociedad Española de Ciencias Hortícolas (SECH).

1. Ávila-Dávila L., Soler-Méndez M., Parras-Burgos D., Bautista-Capetillo C.F., Ruiz-Canales A. y Molina-Martínez J.M. Estimación de la velocidad de infiltración del agua en suelos no saturados empleando lisimetría de pesada. Actas Portuguesas de Horticultura. 2020, 34, 79-85. ISBN: 978-972-8936-36-5.

2. Ávila-Dávila L., Soler-Méndez M., Parras-Burgos D., González-Trinidad J., and Ruiz-Canales A. y Molina-Martínez J.M. Gestión del riego por medio de la velocidad de infiltración del agua en el suelo. Actas Portuguesas de Horticultura. 2020, 34, 8690. ISBN: 978-972-8936-36-5.

3. Escarabajal Henarejos, D., Molina Martínez, J.M., Ávila Dávila, L., y Parras Burgos, D. Influencia de la temperatura en el boro obtenido en aguas desaladas. Actas Portuguesas de Horticultura. 2020, 34, 166-169. ISBN: 978-972-8936-36-5

4. Escarabajal Henarejos, D., Molina Martínez, J.M., Guzmán Raja, I., Ávila Dávila, L., y Parras Burgos, D. Estudio energético del control de la concentración de boro en aguas desaladas mediante regulación de la temperatura. Actas Portuguesas de Horticultura. 2020, 34, 170-173. ISBN: 978-972-8936-36-5. 
5. Ruíz-Peñalver L., Ávila-Dávila L., Soler-Méndez M., Ruiz-Lozano L., y Molina-Martínez J.M. Deriva en la medida del peso por la temperatura en las células de carga y su posible compensación por software. Actas Portuguesas de Horticultura. 2020, 34, 357-364. ISBN: 978-972-8936-36-5.

6. Ruíz-Peñalver L., Soler-Méndez M., Ruiz-Lozano L., Ávila-Dávila L. y Molina-Martínez J.M. Metodología de filtrado de datos en equipos de lisimetría de pesada. Actas Portuguesas de Horticultura. 2020, 34, 140-148. ISBN: 978-972-893636-5.

7. Parras Burgos, D., Soler Méndez, M., Ávila Dávila, L., y Molina Martínez, J.M. Diseño estructural de un equipo de ferticontrol basado en lisimetría de pesada. Actas Portuguesas de Horticultura. 2020, 34, 128-133. ISBN: 978-972-8936-36-5.

8. Parras Burgos, D., Polhmann Barbosa, T., Molina Martínez, J.M., Soler Méndez, M., Ávila Dávila, L., y Fernández-Pacheco, D.G. Uso de la realidad aumentada como complemento formativo en estudios de agronomía. Actas Portuguesas de Horticultura. 2020, 34, 186-189. ISBN: 978-972-8936-36-5.

9. Soler-Méndez, M., Ávila-Dávila, L., Parras-Burgos, D., Ruiz-Lozano, L., Ruiz-Canales, A., Molina-Martínez, J.M. Estandarización de las dimensiones de un lisímetro de pesada de bajo coste para los principales cultivos hortícolas del sureste español. Actas Portuguesas de Horticultura. 2020, 34, 18-25. ISBN: 978-972-893636-5.

10. Soler-Méndez, M., Ávila-Dávila, L., Parras-Burgos, D., Ruiz-Lozano, L., Ruiz-Canales, A., Molina-Martínez, J.M. Propuesta de plataforma de gestión global del riego y los nutrientes. Actas Portuguesas de Horticultura. 2020, 34, 149-156. ISBN: 978-972-8936-36-5

\subsubsection{Congreso de Jóvenes Investigadores en Ciencias Agroalimentarias}

Celebrado el 17 de octubre de 2019 en la Universidad de Almería, en Almería, España.

1. Ávila-Dávila L., Soler-Méndez M., Bautista-Capetillo C.F., González-Trinidad J., y Molina-Martínez J.M. Estudio y evaluación de modelos de gestión de los recursos 
hídricos en agricultura mediante lisimetría. Libro de Actas del II Congreso de Jóvenes Investigadores en Ciencias Agroalimentarias. 2019, p. 242. ISBN: 978-84-09-175475.

2. Soler-Méndez M., Molina-Martínez J.M., Ávila-Dávila L., Ruiz-Peñalver L. Sistema de inteligencia artificial para la gestión de la fertirrigación mediante redes de lisimetría de pesada y sensores agronómicos. Libro de Actas del II Congreso de Jóvenes Investigadores en Ciencias Agroalimentarias. 2019, p.274. ISBN 978-84-0917547-5.

\subsubsection{Congreso Universitario en Innovación y Sostenibilidad Agroalimentaria (CUISA 2020)}

Celebrado el 24 al 25 de septiembre de 2020 en la Escuela Politécnica Superior de Orihuela de la Universidad Miguel Hernández en Orihuela, España.

1. Ávila-Dávila L., Soler-Méndez M., Escarabajal-Henarejos D. y Molina-Martínez J.M. Estimación de la lluvia efectiva mediante utilización de lisimetría de pesada. Actas del I Congreso Universitario en Innovación y Sostenibilidad agroalimentaria. 2020, 310-315. ISBN: 978-84-16024-90-2.

\subsection{Artículos en revistas indexadas como coautora durante el doctorado}

1. González-Trinidad, J.; Júnez-Ferreira, H.E.; Bautista-Capetillo, C.; Ávila Dávila, L.; Robles Rovelo, C.O. Improving the Water-Use Efficiency and the Agricultural Productivity: An Application Case in a Modernized Semiarid Region in North-Central Mexico. Sustainability 2020, 12, 8122. http://doi:10.3390/su12198122

2. Escarabajal-Henarejos, D.; Parras-Burgos, D.; Ávila-Dávila, L.; Cánovas-Rodríguez, F.J.; Molina-Martínez, J.M. Study of the Influence of Temperature on Boron Concentration Estimation in Desalinated Seawater for Agricultural Irrigation. Water 2021, 13, 322. https://doi.org/10.3390/w13030322 


\subsection{Artículos en revistas indexadas como autora}

\subsubsection{Publicación 1}

Notificación de aceptación de la publicación

De: $\quad$ Sustainability Editorial Office <sustainability@mdpi.com>

Recibido el: $\quad$ 09/01/2021

Para: $\quad$ Ávila Dávila <laura_14avila@uaz.edu.mx>

José Miguel Molina-Martínez <josem.molina@upct.es>, Carlos Bautista-Capetillo<baucap@uaz.edu.mx>,

CC: $\quad$ Manuel Soler-Méndez $<$ manuel.ia@agrosolmen.es>, Cruz Octavio Robles Rovelo<corr03@hotmail.com>, Hugo Enrique Júnez-Ferreira <hejunez@uaz.edu.mx>, Julián González-Trinidad <jgonza@uaz.edu.mx>, Asunto: [Sustainability] Manuscript ID: sustainability-1034032 - Accepted for Publication

Dear Mrs. Davila,

We are pleased to inform you that the following paper has been officially accepted for publication:

Manuscript ID: sustainability-1034032

Type of manuscript: Article

Title: Estimation of Evapotranspiration and Crop Coefficients of Bell Pepper Using a Removable Weighing Lysimeter: study case in Southeast of Spain

Authors: Laura Ávila-Dávila *, José Miguel Molina-Martínez, Carlos Bautista-Capetillo *, Manuel Soler-Méndez, Cruz Octavio Robles Rovelo, Hugo Enrique Júnez-Ferreira, Julián González-Trinidad

Received: 3 December 2020

E-mails: laura_14avila@uaz.edu.mx, josem.molina@upct.es, baucap@uaz.edu.mx, manuel.ia@agrosolmen.es,_corr03@hotmail.com, hejunez@uaz.edu.mx, jgonza@uaz.edu.mx

Sustainable Applications in Agriculture

https://www.mdpi.com/journal/sustainability/special_issues/Sustainable_Applications_Agri culture

https://susy.mdpi.com/user/manuscripts/review_info/b03fc4c61344f65715e3b503d8c70d9f

We will now make the final preparations for publication, then return the manuscript to you for your approval. 
If, however, extensive English edits are required to your manuscript, we will need to return the paper requesting improvements throughout.

We encourage you to set up your profile at SciProfiles.com, MDPI's researcher network platform. Articles you publish with MDPI will be linked to your SciProfiles page, where colleagues and peers will be able to see all of your publications, citations, as well as your other academic contributions.

We also invite you to contribute to Encyclopedia (https://encyclopedia.pub), a scholarly platform providing accurate information about the latest research results. You can adapt parts of your paper to provide valuable reference information for others in the field.

Kind regards,

Susan Guo

Assistant Editor

Email: Susan.guo@mdpi.com

MDPI Branch Office, Tianjin

Sustainability Editorial Office

E-mail: sustainability@mdpi.com

http://www.mdpi.com/journal/sustainability/

MDPI

St. Alban-Anlage 66, 4052 Basel, Postfach, CH-4020 Basel, Switzerland 
Article

\title{
Estimation of the Evapotranspiration and Crop Coefficients of Bell Pepper Using a Removable Weighing Lysimeter: A Case Study in the Southeast of Spain
}

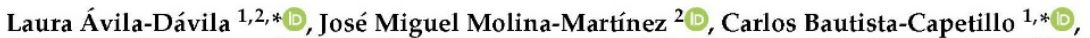 \\ Manuel Soler-Méndez ${ }^{2}$, Cruz Octavio Robles Rovelo ${ }^{1}{ }^{\circledR}$, Hugo Enrique Júnez-Ferreira ${ }^{1}(\mathbb{D}$ and \\ Julián González-Trinidad ${ }^{1}$ (1) \\ 1 Doctorado en Ciencias de la Ingeniería, Universidad Autónoma de Zacatecas, Zacatecas 98160, Mexico \\ corr03@hotmail.com (C.O.R.R.); hejunez@uaz.edu.mx (H.E.J.-F.); jgonza@uaz.edu.mx (J.G.-T.) \\ 2 Research Group Ingeniería Agromótica y del Mar, Universidad Politécnica de Cartagena, 30203 Cartagena, \\ Spain; josem.molina@upct.es (J.M.M.-M.); manuel.ia@agrosolmen.es (M.S.-M.) \\ * Correspondence: laura_14avila@uaz.edu.mx (L.Á.-D.); baucap@uaz.edu.mx (C.B.-C.); \\ Tel.: +52-492-106-4540 (C.B.-C.)
}

check for
updates

Citation: Ávila-Dávila, L.

Molina-Martínez, J.M.

Bautista-Capetillo, C.; Soler-Méndez,

M.; Robles Rovelo, C.O.;

Júnez-Ferreira, H.E.

González-Trinidad, J. Estimation of

the Evapotranspiration and Crop

Coefficients of Bell Pepper Using a

Removable Weighing Lysimeter: A

Case Study in the Southeast of Spain.

Sustainability 2021, 13, 747.

https://doi.org/10.3390/su13020747

Received: 3 December 2020

Accepted: 10 January 2021

Published: 14 January 2021

Publisher's Note: MDPI stays neutral with regard to jurisdictional clai$\mathrm{ms}$ in published maps and institutional affiliations.

\section{(c) (1)}

Copyright: $(2021$ by the authors. Licensee MDPI, Basel, Switzerland. This article is an open access article distributed under the terms and conditions of the Creative Commons Attribution (CC BY) license (https:// creativecommons.org/licenses/by/ $4.0 /$ )
Abstract: Water use efficiency is essential in semiarid regions of Spain, and it can be achieved through a precise knowledge of the real crop water requirements (CWR). 'The Food and Agriculture Organization of the United Nations (FAO) offers standardized crop coefficients to establish the CWR. However, these coefficients can change due to different conditions, such as climatic variations and cultivation practices. In this work, the evapotranspiration $\left(E T_{C_{l y s}}\right)$ and crop coefficients $\left(K_{C_{l y s}}\right)$ of bell pepper were obtained with a compact removable weighing lysimeter between February and August for two crop seasons (2019 and 2020). $L T_{C_{i y s}}$ was determined from the water balance, and the $K_{C_{i y s}}$ values were determined as the ratio of the crop evapotranspiration, measured on the removable weighing lysimeter, and the reference evapotranspiration. The $K_{C_{i y s}}$ average values for the bell pepper in the initial, middle, and final stages were $0.57,1.06$, and 0.80 , respectively. $K_{C}$ regression models were obtained as a function of the fraction thermal units, achieving a maximum correlation of $0.67\left(R^{2}\right)$. In general, the $K_{C}$ values obtained in this research work were lower in the initial and in the final stages and larger in the middle stage in comparison with the FAO-56 values and other research works values in semiarid conditions. The bell pepper yield increased by $7.72 \%$ in 2019 and by $3.49 \%$ in 2020 compared to the yield reported by the Ministry of the Environment and Rural and Marine Areas of the Spanish Government in 2019 and with a minimum water loss through drainage. The results in this work can help farmers to determine the crop water requirements and to improve the system efficiency in semiarid locations with similar conditions to those in the study.

Keywords: mini-lysimeter; thermal units; horticultural crop evapotranspiration; semiarid conditions

\section{Introduction}

The irrigation areas in southeast Spain are characterized by limited water resources due to a semiarid climate. 'The Mediterranean basin has very hot summers that increase the crop water demand via an increase in the crop evapotranspiration rate [1-3]. Insufficient water for crop management causes poor water distribution and a reduction in agricultural yield, which is associated with less growth and crop development $[4,5]$; therefore, strategies must be adopted in order to optimize water resources and to obtain profitable crops [1].

In the Mediterranean area, Andalusia, Murcia, and Valencia are the three main regions of horticultural production, mostly involving spring-summer crops. The bell pepper production in this area mainly occurs in the Murcia region, where greenhouses reach up to $82 \%$ of the total cultivated hectares while open-field cultivation represents only $18 \%$. The average greenhouse yield is $30 \%$ higher than the yield of open fields [6]. According to 
Nikolau et al. [7], the use of greenhouses allows a more efficient water use in comparison to open-field cultivation because the plastic covering reduces soil evaporation, with a $38 \%$ reduction in the water requirements of bell pepper.

In agricultural areas with limited water resources, such as the Mediterranean area, it is common to use a drip irrigation system due to its punctual application [5]; however, if the irrigation system is not properly managed, there will be water losses, usually due to run-off or water leaks in the conduction system itself.

In order to achieve the optimal irrigation application and to maximize its efficiency, it is necessary to know the precise amount of water required by the crop for its specific development conditions $[4,8]$. These water requirements can be estimated accurately through a water balance, which quantifies the input and output flows of the soil profile in the crop root zone [8-10]; in addition, a water balance helps to reduce water losses and to diagnose the irrigation method itself [11].

There are different and indirect methodologies for estimation of the crop water requirements based on the crop evapotranspiration $\left(E T_{C}\right)$, such as the standard approach proposed by the Food and Agriculture Organization of the United Nations (FAO), which uses the concept of the reference evapotranspiration $\left(E T_{O}\right)$ and crop coefficient $\left(K_{C}\right)$. This methodology has two steps. The first uses climatic variables such as the relative air humidity, the sunshine hours, the solar radiation, the wind speed, and the air temperature in order to obtain the $E T_{O}$ that refers to a uniformly growing crop, similar to a field of green grass, well irrigated and with a uniform height. This is called the FAO Penman-Monteith method and represents the effect of climate on water requirements [12].

The second step refers to the $K c$ that represents the effect of the crop growth with time as a coefficient. For this coefficient, the FAO proposes two approaches in order to integrate the physical and physiological crop properties. The first approach is called single coefficient and integrates the $E T_{O}$ and the $E T_{C}\left(K c=E T_{C} / E T_{O}\right)$, while the second approach separates evaporation and transpiration. There are different ways to measure the $K_{C}$. One method considers the crop stress through the fraction thermal units or growth degree days from the average air temperature. Fraction thermal units measure the accumulated heat above the base temperature, which is different between crop varieties; plant growth is zero when the temperature is below the base temperature $[13,14]$. Similarly, $K_{C}$ can be determined in the days after planting (DAP) [13] from vegetation indexes and satellite image processing $[15,16]$, or as a percentage of green coverage via the processing of digital photos [2].

In this context, a weighing lysimeter provides a direct method to measure the $E T_{C}$ through the mass balance of an isolated soil volume [17]. The use of weighing lysimetry in the $E T_{C}$ estimation has been widely studied [3,18-21], and it has also been used to study the flows involved in the water balance and solutes in soil profiles under different conditions [22], such as dew, fog and frost [23], precipitation [24-28], and percolation [29]. One of the main features of such a device is the high accuracy, allowing its use to establish and calibrate different mathematical models $[9,17]$.

Weighing lysimeters usually have large dimensions and require a large set-up area and specialized maintenance, making them expensive and of limited use for research purposes. The results from lysimetry research can be used by farmers when the conditions are similar to those in the experiment [30]. Ruiz-Peñalver et al. [30] and Nicolás-Cuevas et al. [31] developed compact and transportable weighing lysimeters that can be used in commercial plots to evaluate the irrigation application efficiencies or for estimating the crop water requirements; once the harvest is finished, the lysimeters are easy to move. Other small commercial lysimeters have been produced by the METER Group ${ }^{\circledR}$ (USA) [32] and UGT Company (Germany) [33].

The objective of this work was to compute the evapotranspiration of a bell pepper crop by using a removable weighing lysimeter during two crop seasons and to determine the crop coefficients adapted to the specific climatic and technical conditions. The results were compared to those obtained with the FAO-56 methodology, with the $K_{C}$ inferred from 
the lysimeter being used to establish the relationship as a function of the thermal units. The crop yield was also evaluated.

\section{Materials and Methods}

2.1. Study Area

The experiment was conducted during 2019 and 2020 in a commercial bell pepper plot (Capsicum annuum L. var. Maestral), located in San Javier in the Murcia region in the southeast of Spain (Figure 1a), with the geographic coordinates $37^{\circ} 51^{\prime} 11.80^{\prime \prime} \mathrm{N}$ latitude and $0^{\circ} 49^{\prime} 50.00^{\prime \prime} \mathrm{W}$ longitude and an altitude of 15 m.a.s.l. (meters above the soil level). The experimental plot was an open field, and the area around the plot was dominated by greenhouses. The plot is highlighted in red in Figure 1b. The predominant climate in the study area has been identified as Mediterranean subdesert, with maximum and minimum temperatures of 39.6 and $12.9^{\circ} \mathrm{C}$, respectively, and an average annual rainfall of $313 \mathrm{~mm}[2]$.

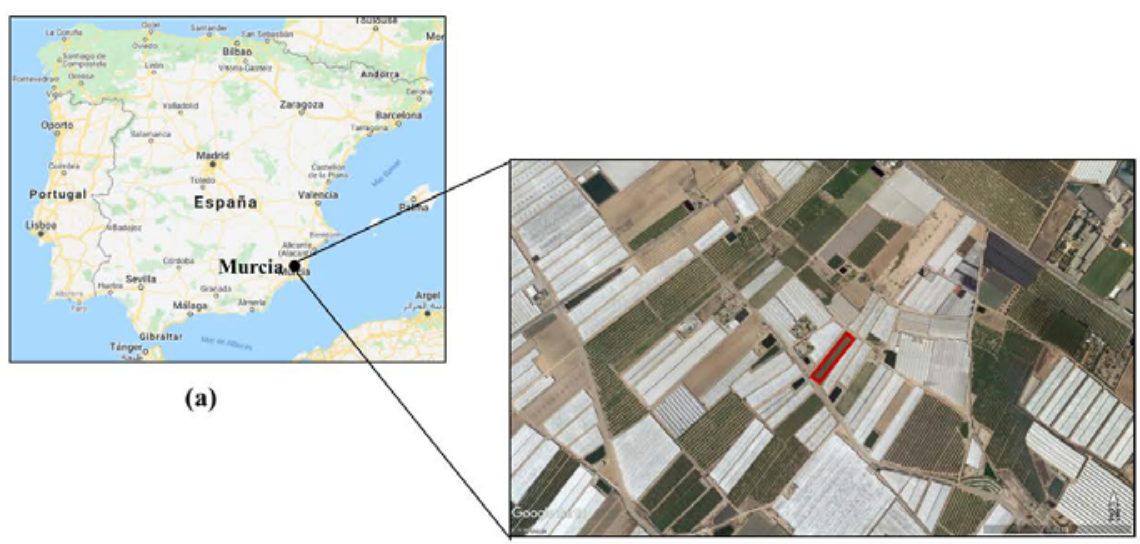

(b)

Figure 1. Study area: (a) Region of Murcia and (b) location of the experimental plot.

\subsection{Description of the Removable Weighing Lysimeter}

The removable weighing lysimeter consists of two recipients. One of them is the cultivation recipient (CR) that contains the soil profile and the crop. It has the dimensions of $0.56 \times 0.96 \times 0.30 \mathrm{~m}$ with a small slope at the bottom along all four sides of the container, so that the drained water is guided by gravity to a central hole at the bottom to avoid water accumulation. The second is the drainage recipient (DR) that stores the water that vertically drains from the $C R$, similar to the one used by Nicolás Cuevas et al. [31].

The lysimeter was installed in the center of the plot, avoiding the edges, because it was necessary that it be surrounded by the same crop to obtain reliable measurements [34]. In a previously excavated hole, in which the dimensions of the lysimeter were considered, the soil extracted during the excavation was then placed in the CR, while trying to keep the soil unaltered. A hydrodynamic characterization of the clay soil was performed based on the methodology proposed by the U.S. Department of Agriculture (USDA) [35] with a bulk density of $1.38 \mathrm{~g} / \mathrm{cm}^{3}$. The irrigation system used was drip irrigation, with emitters placed every $25 \mathrm{~cm}$ and an application rate of $2.2 \mathrm{~L} / \mathrm{h}$ (Figure 2). 


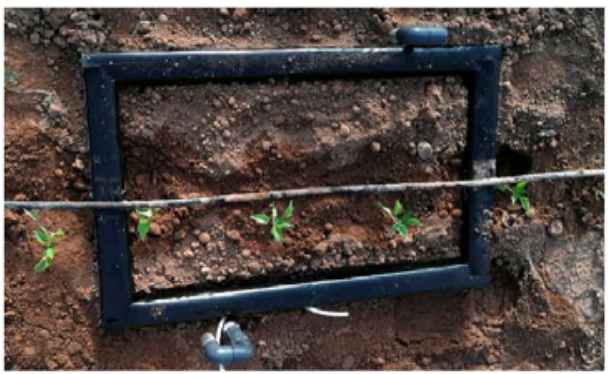

Figure 2. Top view of the compact weighing lysimeter during the experimentation.

\subsection{Crop Management}

The bell pepper crop (Capsicum annuum L.), previously sowed under greenhouse conditions, was transplanted at $10 \mathrm{~cm}$ height. The planting frame measured $1 \mathrm{~m}$ between lines and $0.33 \mathrm{~m}$ between plants, leaving a total of three plants and three drippers within the CR (Figure 2). The vegetative cycle until the harvest was 195 days. The irrigation scheduling was performed following the $E T_{C}$ computation with the lysimeter and with $91 \%$ application efficiency.

The fertilization dose was applied with the irrigation water according to the recommendations of the Ministry of Agriculture, Fisheries and Food of Spain [36]; meanwhile, weeds and pests were managed according to the common practices in the agricultural area.

\subsection{Determination of Evapotranspiration and Crop Coefficients}

The computation of evapotranspiration started with the crop transplanting. Then, through the application of irrigation, the soil reached field capacity, with the initial value of CR representing the soil mass plus water mass plus the mass of three plants. During the crop development, if the soil did not receive water, the CR mass decreased because of the $E T_{C}$. When irrigation was applied or rainfall occurred, the RC's mass increased rapidly; if the soil field capacity was exceeded, the soil began to drain or discharge the excess water from the bottom of the RC, which was reflected by the increased mass of the DR. Once irrigation or rainfall was stopped, the soil lost water because of the $E T_{C}$.

From the operation behavior of the lysimeter described above, the daily crop evapotranspiration was calculated by applying the water balance equation [37] and the conditions established in the work of Peters et al. [28], as in Equations (1) and (2):

$$
E T_{C_{L Y S}}=\mathrm{I}-\Delta \mathrm{DR} \pm \Delta \mathrm{CR}
$$

Under conditions of no rain or irrigation water, Equation (1) would be as follows:

$$
E T_{C_{L Y S}}=-\Delta C R-\Delta \mathrm{DR}
$$

where $I$ is the irrigation depth ( $\mathrm{mm}), \triangle \mathrm{CR}$ is the mass variation in the cultivation recipient $(\mathrm{mm})$, and $\triangle \mathrm{DR}$ in the mass increase in the drainage recipient $(\mathrm{mm})$, considering that $1 \mathrm{~kg}$ of lysimeter variation represents $1.85 \mathrm{~mm}$ of water.

From the data obtained with the lysimeter, the daily values of the crop coefficient $\left(K_{C_{l y s}}\right)$ were calculated using Equation (3) [12]:

$$
K_{C l y s}=\frac{E T_{C_{L Y S}}}{E T_{O}}
$$

where $E T_{O}$ is the reference evapotranspiration estimated by the FAO Penman-Monteith method [12], considering the climate records generated in an automatic weather station built with a datalogger (CR10X model, Campbell Scientific, Logan, UT, USA), a pyranome- 
ter (CM14 model, KIPP\&ZONEN Delft), temperature and relative humidity sensors placed at $1.5 \mathrm{~m}$ above the ground level (MP100 probe, Campbell Scientific, Logan, UT, USA), anemometer and anemovelta at a $2 \mathrm{~m}$ height (A100R and W200P models, respectively, of Vector Instruments Ltd., Rhyl, North Wales, UK), and a rain gauge (ARG100 model, Campbell Scientific, Logan, UT, USA). The automatic weather station was installed next to the weighing lysimeter. The phenological stages were defined according to the Allen et al. [12]'s recommendations.

The $E T_{C}$ value determined with the FAO-56 method [12] was compared with the $E T_{C}$ obtained with the lysimeter, and the model used was as follows:

$$
E T_{C_{F A O}}=E T_{O} \times K_{C_{F A O}}
$$

where $K_{C_{F A O}}$ refers the values proposed by the FAO-56 for bell pepper for the different stages of crop development.

From the average air temperature obtained with the weather station, the crop thermal units $(T U)$ were determined for the whole cycle [14] by considering a $10^{\circ} \mathrm{C}$ basal temperature $\left(T_{b}\right)$, proposed by Vidal [38], which is the minimum temperature at which the bell pepper can develop.

$$
\begin{array}{cc}
\text { If } T_{a}>T_{b}: & T U_{i}=\left(T_{a}-T_{b}\right)_{i} \\
\text { If } T_{a} \leq T_{b}: & T U_{i}=T U_{i-1}
\end{array}
$$

where $T U_{i}$ is the thermal unit for day $i\left({ }^{\circ} \mathrm{C}\right), T U_{i-1}$ is the thermal unit for day $i-1\left({ }^{\circ} \mathrm{C}\right)$, and $T_{a}$ is the average air temperature in $\left({ }^{\circ} \mathrm{C}\right)$ for day $i$.

The $T U$ values were converted to accumulated fraction thermal units $(F T U)$ :

$$
F T U_{i}=\frac{T U_{i}}{\Sigma T U_{i}}
$$

where $\Sigma T U_{i}$ is the total accumulative $T U$ for optimal growth of the bell pepper, proposed by Vidal to be $2200 \pm 220^{\circ} \mathrm{C}$ [38]. In this experiment, the total cumulative thermal units for the 2019 and 2020 seasons were 1860 and $1904{ }^{\circ} \mathrm{C}$, respectively. A relationship was obtained for the FTU results and the crop coefficient from the lysimeter using the least squares algorithm [39].

Different metrics were used to compare the results obtained from the different methodologies, such as the mean estimation error (MEE), the root mean square error (RMSE), the systematic mean square error $\left(\mathrm{MSE}_{s}\right)$, the coefficient of determination $\left(R^{2}\right)$, and the index of agreement (LA).

$$
\begin{gathered}
\text { MEE }=\frac{1}{n} \sum_{i=1}^{n} Y_{i}-\hat{Y}_{i} \\
\text { RMSE }=\sqrt{\frac{1}{n} \sum_{i=1}^{n}\left(Y_{i}-\hat{Y}_{i}\right)^{2}} \\
\text { MSE }_{s}=\frac{1}{n} \sum_{i=1}^{n}\left(\hat{Y}_{i}-Y_{i}\right)^{2} \\
\mathrm{LA}=1-\frac{\sum_{i=1}^{n}\left(\mathrm{y}-\hat{Y}_{i}\right)^{2}}{\sum_{i=1}^{n}(|\hat{Y}-\bar{y}|+|y-\bar{y}|)^{2}}
\end{gathered}
$$

where $n$ is the data number, $Y_{i}$ is the value measured of $i, \hat{Y}_{i}$ is the estimated value in the regression of day $i$ or value to be compared, and $\bar{y}$ is the mean of the measured values of day $i[40,41]$. 


\subsection{Water Productivity}

According to the methodology proposed by Playán and Mateos [42], the water productivity was determined by the relationship between the production obtained and the volume consumed, expressed in $\mathrm{kg} / \mathrm{m}^{3}$, which related to the crop yield per cultivated area in $t / h a$ and the economic benefits per cultivated area, EUR/ha.

3. Results and Discussion

\subsection{Meteorological Conditions}

The 2019 growing season was from 15 February to 28 August, while the 2020 season was from 10 February to 22 August, both from the date of transplant. The 2019 and 2020 average air temperature, average relative humidity, average wind speed, and $E T_{O}$ for the different periods are shown in Figure 3. The behavior of the climatic variables was very similar for the two seasons. Comparing the two years, March, the end of April, and the first days of June were warmer in 2020, while late June and early July were warmer in 2019.
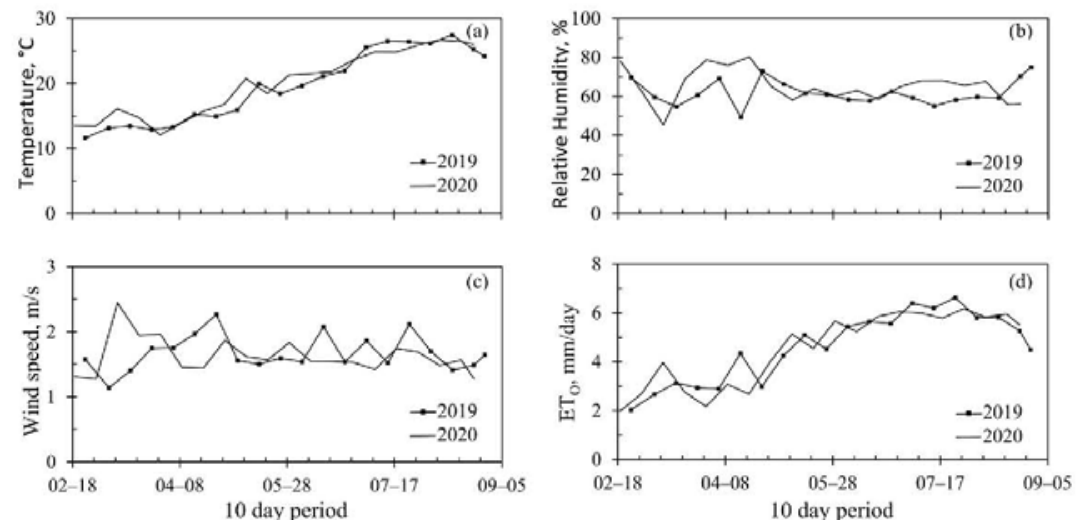

Figure 3. Average meteorological data for a 10-day period: (a) ambient temperature, (b) relative humidity, (c) wind speed, and (d) reference evapotranspiration $\left(E T_{O}\right)$.

The relative humidity and wind speed were lower in 2019 than in 2020 for most of the growing season. The relative humidity was especially lower from 14 March to $18 \mathrm{April}$ and from 27 July to 10 August in 2020 . The average relative humidity values recorded for the two seasons were greater than $60 \%$, with minimum and maximum averages of $47.31 \%$ and $76.61 \%$, respectively. The average wind speed was $1.67 \mathrm{~m} / \mathrm{s}$ in 2019 and $1.65 \mathrm{~m} / \mathrm{s}$ in 2020 . The $E T_{O}$ increased in a similar manner to the ambient temperature, with the average $E T_{O}$ being $4.6 \mathrm{~mm} /$ day in 2019 and $4.6 \mathrm{~mm}$ /day in 2020 .

\subsection{Crop Evapotranspiration}

Figure 4 shows the daily values of the $E T_{C}$ of the crop, the inputs due to rain and/or irrigation, and the water losses by drainage. Initially, irrigation was applied every three days on average; however, according to the crop growth and an increase in $E T_{C}$ as a result of high summer temperatures, the schedule changed to every two days, then decreased again to one day between irrigations. 

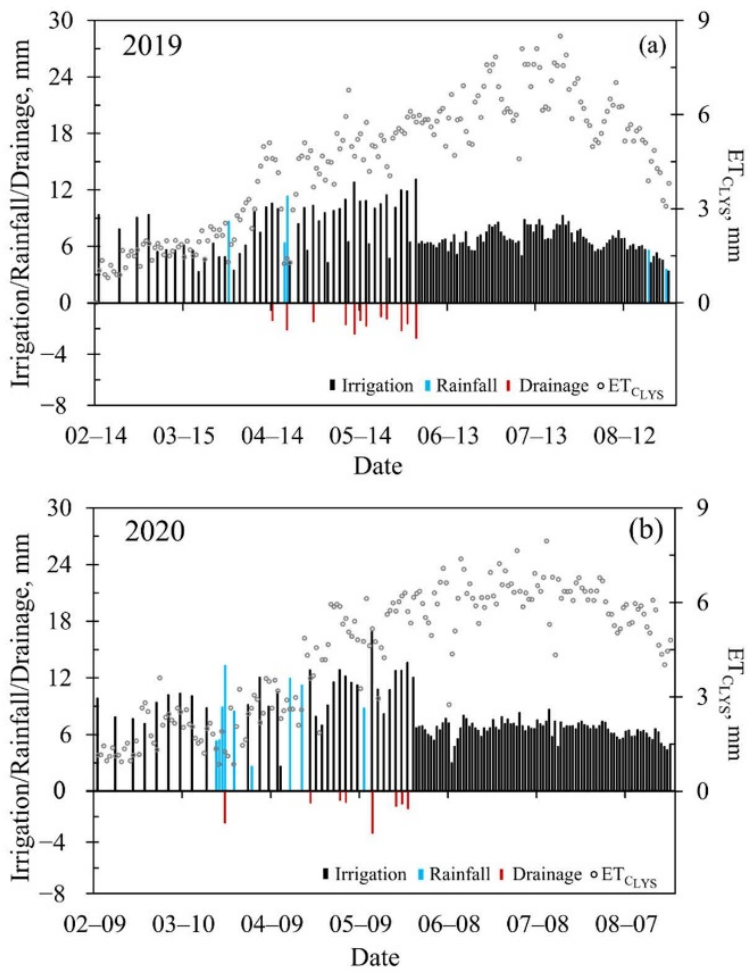

Figure 4. Daily evapotranspiration of the bell pepper measured with the weighing lysimeter $\left(E T_{C_{I Y S}}\right)$ and irrigation, rainfall, and drainage: (a) 2019 and (b) 2020.

The irrigation intervals showed a similar trend in both seasons; the widest interval between irrigations for the 2019 season was five days and extended only until February, while for the 2020 season, the widest interval was three days on average and extended until 20 April. In April, the $E T_{C}$ increased, causing the interval to decrease to two days. In the days at the end of May, when summer started, irrigation took place daily. In this period, the daily evapotranspiration of the crop was higher than $5 \mathrm{~mm}$. This interval started earlier in the 2020 season. The total application of irrigation was $960.3 \mathrm{~mm}$ in 2019 and $936.1 \mathrm{~mm}$ in 2020. In 2020, there were more rainfall events and therefore less irrigation was applied, with $40.7 \mathrm{~mm}$ more rain and double the number of events, meaning $2.52 \%$ less irrigation applied.

Table 1 shows the average values for irrigation, rainfall, drainage, and evapotranspiration for both years.

Table 1. Seasonal water balance in millimeters.

\begin{tabular}{ccccc}
\hline Season & Irrigation & Rainfall & Drainage & $E_{C_{L \text { LS }}}$ \\
\hline 2019 & 960.3 & 35.4 & 12.31 & 874.4 \\
2020 & 936.1 & 76.1 & 12.03 & 854.6 \\
\hline
\end{tabular}

Comparing the gross depth of irrigation obtained with the lysimeter to that obtained with the FAO-56 methodology, a decrease of $1.33 \%$ was found in 2019 because the water 
depth was $947.6 \mathrm{~mm}$ with FAO. In 2020, the water depth was $928.8 \mathrm{~mm}$, representing a decrease of $0.78 \%$ with respect to the lysimeter measurements. These decreases were small, and the difference in depth can mean just one or two irrigations. The drainages reported by the lysimeter achieved an average of $12 \mathrm{~mm}$, with 12 and 14 events for the 2019 and 2020 seasons, respectively. In the first season, the drainages did not exceed $4 \mathrm{~mm}$ and were caused by the irrigations that came to exceed a $10 \mathrm{~mm}$ depth; for instance, the drainage caused by the rains on 19-21 April with a $28.4 \mathrm{~mm}$ depth. In the second season, one of the drainages was caused by rain on 21-24 March of $33 \mathrm{~mm}$ in depth and the others were caused by an irrigation depth larger than $12 \mathrm{~mm}$. The major rainfall events were in the months of March and April; for the first year, there were five days of rain with an accumulated depth of $35.4 \mathrm{~mm}$, while in the second year, there were double the number of days and an accumulated depth of $76.1 \mathrm{~mm}$.

The $E T_{C}$ increased when the temperature increased and the interval between irrigations decreased, indicating the relationship that $E T_{C}$ has with both variables. The maximum $E T_{C}$ value was reached close to 16 July for the both seasons. When the irrigation application was on a daily scale, the $E T_{C}$ had high values above $5 \mathrm{~mm}$. The behavior of the $E T_{C}$ during rainy days was small, and it was larger the day after irrigation or rain because the evaporation of the wet soil was more significant; in this same context, in the days when no irrigation was performed, due to longer intervals, the $E T_{C}$ was maintained or declined, because there is less evaporation from a soil with a dry surface [12]. The total $E T_{C}$ for the 2019 and 2020 seasons were $874.4 \mathrm{~mm}$ and $854.6 \mathrm{~mm}$, respectively.

The lysimeter-measured $E T_{C_{L Y S}}$ values for both seasons were compared to the $E T_{O}$ calculated using the Penman-Monteith method and the $E T_{C}$ obtained using the method established by the standard methodology of FAO-56 $\left(E T_{C_{F A O}}\right)$ [12], presented in Figure 5. The resulting average $E T_{C_{L Y S}}$ values for the initial, middle, and final stages were $1.6,5.8$, and $5.4 \mathrm{~mm} /$ day, respectively.

The linear correlation between $E T_{C_{t \gamma s}}$ and $E T_{O}$ showed a good agreement (Figure 5b). The determination coefficient was 0.90 and the RMSE was $1.04 \mathrm{~mm} /$ day for both seasons, indicating the dependence that exists between the variables. In the comparison of $E T_{C_{L Y S}}$ and $E T_{C_{F A O}}$, the linear regression slope was close to unity and the RMSE was $0.63 \mathrm{~mm} /$ day. The values of $E T_{C_{F A O}}$ in the whole crop cycle overestimated the $E T_{C_{I Y S}}$ by $37.44 \%$, with an average value of $0.4 \mathrm{~mm}$ /day; this may be due to the fact that the $K_{C}$ values used in the FAO-56 methodology were standard values calibrated under different conditions than those of this experiment.

The MEE was close to zero $(0.03 \mathrm{~mm} /$ day in 2019 and $0.01 \mathrm{~mm} /$ day in 2020), indicating a low bias between $E T_{C_{L Y S}}$ and $E T_{C_{F A O}}$. Similarly, the other metrics showed the same behavior. The IA values were close to one ( 0.98 in 2019 and 0.98 in 2020), indicating a good agreement with both $E T_{C}$ models. However, the $\mathrm{MSE}_{\mathcal{S}}$ values were $4.05 \%$ in 2019 and $3.91 \%$ in 2020 , indicating high bias, meaning one of the methods should be improved. The MSEs obtained by Martínez-Cob [14] are very similar to the results of this research, with both $\mathrm{MSE}_{\mathrm{S}}$ being greater than two.

\subsection{Crop Coefficient}

The crop coefficient values estimated from Equation (3) $\left(K_{C_{\text {lys }}}\right)$ and the values established in the FAO-56 paper for this crop $\left(K_{C_{F A O}}\right)$ are show in Figure 6 . The $K_{C_{l y s} \text { ini }}$ of the lysimeter was $0.57, K_{C_{\text {lys }} \text { mid }}$ was 1.06 , and $K_{C_{\text {lys }} \text { end }}$ was 0.80 . These data were adjusted to a third-degree polynomial curve, with an $R^{2}$ of 0.73 . In this model, a maximum $K_{C}$ of 1.11 and a minimum of 0.48 were observed. 

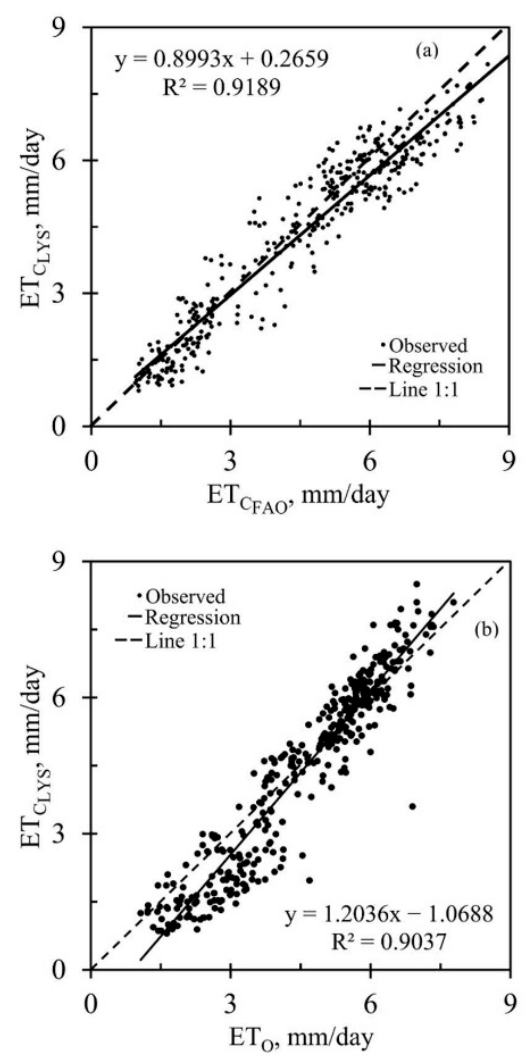

Figure 5. Correlation between $E T_{C L Y S}$ measured by the weighing lysimeter and $E T_{C F A O}$ determined by FAO-56 method (a) and between $E T_{C I Y S}$ and $E T_{O}(\mathbf{b})$.

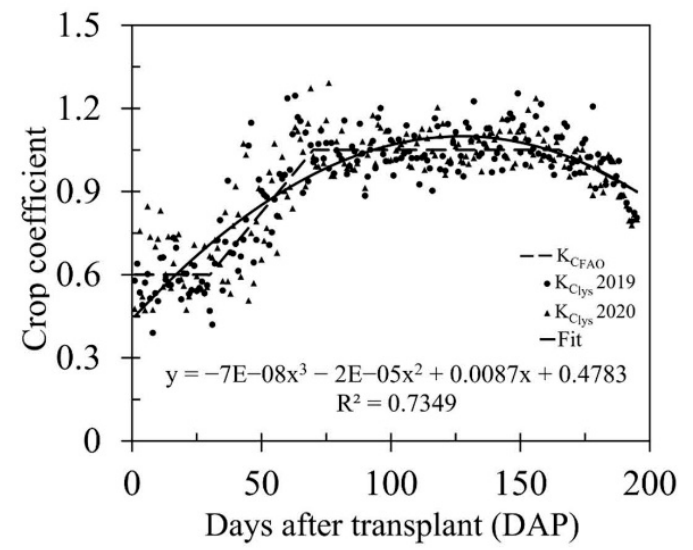

Figure 6. Bell pepper crop coefficients the during the crop cycle (lysimeter and FAO-56 method values). 
When comparing $K_{C_{l y s}}$ to $K_{C_{F A O}}$ an $R^{2}$ of 0.79 and an RMSE of 0.09 were obtained. The values of $K_{C_{l y s}}$ oscillated around the values recommended by Allen et al. [12].

The jump in $K_{C_{\text {lys }}}$ between DAP 50 and 75 (Figure 6) may be due to the low reference evapotranspiration values reported on those days in both seasons, being more significant for the 2020 season. It should be remembered that in Equation (3), $K_{C_{\text {lys }}}$ is a function of $E T_{O}$, so the lower the $E T_{O}$, the higher the $K_{C_{l y g}}$.

A relationship between $K_{C_{\text {lys }}}$ and the fraction thermal units $(F T U)$ was obtained; Figure 7 shows the two resulting graphs of the polynomial fit. As shown in Figure 7a, the maximum $K_{C_{F T U}}$ value was 1.15 for the $F T U$ range of $0.43-0.62$; the maximum for Figure $7 \mathrm{~b}$ was 1.15 in the range of $0.36-0.49$. Although, the second setting (Figure $7 \mathrm{~b}$ ) showed a slightly better $R^{2}$, it reached a maximum curve during the early FTU period and a slower decrease; the curve at the end of the cycle rose and moved away from the observed points.
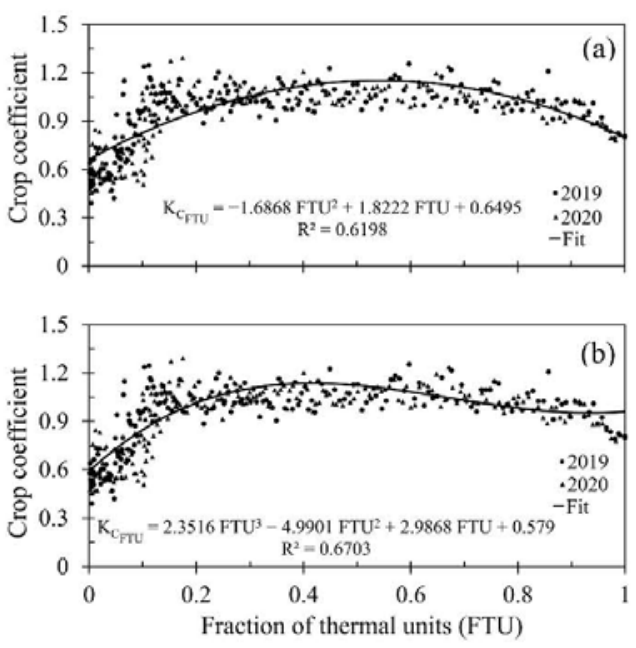

Figure 7. Polynomial fitting of the crop coefficient $\left(K_{C}\right)$ values obtained for 2019 and 2020: (a) Second-order polynomial fitting and (b) third-order polynomial fitting.

The average total of FTU was 1881.76, a value in the range proposed by Vidal [38]. There was a lower bias between $K_{C_{F A O}}$ and $K_{C_{F T U}}$, followed by $K_{C_{F A O}}$ against $K_{C_{\text {lys }}}$ with 0.09 and $K_{C_{F T U}}$ against $K_{C_{l y s}}$ with 0.48. These values are shown in Table 2.

Table 2 shows the $K_{C}$ values of two works that estimated the $K_{C}$ values for the bell pepper. The work of Shukla et al. [43] was performed in Florida, USA during the 2003 2008 fall-winter seasons, with a temperature range of $17-29^{\circ} \mathrm{C}$, an annual rainfall of $1260 \mathrm{~mm} /$ year, a total $E T_{C}$ of $267 \mathrm{~mm}$, and with a high water table. The crop bed was covered with plastic mulch that covered $33 \%$ of the lysimeter area.

In the area of Peninsular Malaysia, characterized by a warm and humid climate, the second study was performed by Muniandy et al. [44] during August 2013 and May 2014 , with an average temperature range of $24-30^{\circ} \mathrm{C}$, a monthly rainfall of $125-270 \mathrm{~mm}$, and relative humidity $(\mathrm{RH})$ between $63 \%$ and $88 \%$. Both investigations used the same methodology to determine the $E T_{C}$ and $K_{C}$ as used in this study.

The temperature range and the relative humidity of both works were in the same range as our work, but with higher rainfalls than those registered in a whole year in our study zone and performed in a different sowing season. Shukla et al. [43] reported high bimonthly values of $E T_{C}$ for their seasons, because irrigation and rainfall kept the groundwater table high and increased the soil moisture with mulching. Therefore, the $K_{C}$ values of this research were the highest (Table 1); they were $50.88 \%, 14.15 \%$, and $60 \%$ 
higher in each stage, respectively, compared to values reported with the lysimeter in this research.

Table 2. $K_{C}$ values obtained in this study and other works.

\begin{tabular}{|c|c|c|c|c|c|c|c|}
\hline \multirow{2}{*}{$K_{C}$ Values } & \multicolumn{3}{|c|}{ Crop Stage } & \multirow{2}{*}{ Location } & \multirow{2}{*}{$\begin{array}{l}\text { Irrigation } \\
\text { Method }\end{array}$} & \multirow{2}{*}{$\begin{array}{c}\text { Climate } \\
\text { Conditions }\end{array}$} & \multirow{2}{*}{$\begin{array}{l}\text { Crop Cycle } \\
\text { (Day) }\end{array}$} \\
\hline & Initial & Middle & End & & & & \\
\hline$K_{C_{\text {lys }}}$ & 0.57 & 1.06 & 0.80 & & & Temp. $12-28^{\circ} \mathrm{C}$ & \\
\hline$K_{\mathrm{C}_{\text {ETU }}}$ * & 0.69 & 1.05 & 0.78 & Murcia, Spain & Drip & RH $47 \%-77 \%$ & 195 \\
\hline$K_{C_{F T U}}{ }^{* *}$ & 0.65 & 1.06 & 0.93 & & & Rain $<100 \mathrm{~mm} /$ season & \\
\hline Allen et al. [12] & 0.6 & 1.05 & 0.9 & $\begin{array}{c}\text { Europe and } \\
\text { Mediterranean }\end{array}$ & - & $\begin{array}{c}E T_{c} \approx 860 \mathrm{~mm} / \text { season } \\
\mathrm{RH} \approx 45 \%\end{array}$ & 125 \\
\hline Shukla et al. [43] & 0.86 & 1.21 & 1.28 & Florida, USA & Sub-surface & $\begin{array}{c}\text { Temp. } 17-29^{\circ} \mathrm{C} \\
\text { Rain } 1260 \mathrm{~mm} / \text { year } \\
E T_{c} 267 \mathrm{~mm}\end{array}$ & 100 \\
\hline Muniandy et al. [44] & 0.67 & 0.95 & 0.76 & $\begin{array}{l}\text { Kluang, } \\
\text { Malaysia }\end{array}$ & Sprinkler & $\begin{array}{c}\text { Temp. } 24-30^{\circ} \mathrm{C} \\
\text { Rain } 125-270 \mathrm{~mm} / \mathrm{month} \\
\text { RH } 63 \%-88 \%\end{array}$ & 125 \\
\hline
\end{tabular}

"Second-order polynomial fit values; " third-order polynomial fit values. $\mathrm{RH}$, relative humidity.

In contrast, the values of Muniandy et al. [44] in the middle and final stages were the lowest, as shown in Table 2. The climatic conditions were very similar to those of the work mentioned above, but without the influence of the water table. These values presented a reduction of $10.38 \%$ and $5 \%$ in $K_{C_{\text {mid }}}$ and $K_{C_{n t d}}$, respectively, from those reported in this work with the lysimeter. The values proposed by Allen et al. [12] presented less variability with respect to those obtained with the lysimeter in this research, with an increase in the initial and final stages of $5.26 \%$ and $12.5 \%$, respectively, and a decrease in the middle stage of $0.94 \%$. The FAO values pertain to climates with a $\mathrm{RH}$ close to $45 \%$ and wind speeds close to $2 \mathrm{~m} / \mathrm{s}$; our conditions satisfy these figures.

3.4. Yield

Table 3 shows a summary of the final production achieved in each season. The bell pepper yield for both seasons was superior to that reported by the Ministry of the Environment and Rural and Marine Areas of the Spanish Government in 2019 of $75.66 \mathrm{t} / \mathrm{ha}$ for the Region of Murcia [6]. The removable weighing lysimeter allowed greater precision in the control of the crop water balance, which increased the crop yield and produced a significant water saving in comparison to the common practices of the Region of Murcia. The water production and economic yields were similar for both seasons.

Table 3. Production data of both seasons.

\begin{tabular}{cccc}
\hline Year & $\begin{array}{c}\text { Bell Pepper Yield } \\
(\mathbf{t} / \mathbf{h a})\end{array}$ & $\begin{array}{c}\text { Water Productivity } \\
\left(\mathrm{Kg}_{\mathbf{m}} \mathbf{m}^{3}\right)\end{array}$ & $\begin{array}{c}\text { Economic Yield } \\
(\text { EUR/ha) }\end{array}$ \\
\hline 2019 & 81.5 & 8.49 & $64,457.55$ \\
2020 & 78.3 & 8.36 & $64,808.91$ \\
\hline
\end{tabular}

In 2019 , a better yield was obtained, as the excess humidity in the soil caused by the rains in 2020 generated an abortion of flowers, reducing the productivity of the bell pepper in that year [45].

\section{Conclusions}

The $E T_{C}$ and $K_{C}$ values for the bell pepper crop were calculated using a compact weighing lysimeter and an automatic weather station for two seasons in the semiarid southeast of Murcia, Spain. The $E T_{C}$ was affected by irrigation and rainfall events, which increased the soil evaporation, most notably when there was a shorter interval between 
irrigations. Second- and third-order models were developed for $K_{C}$ as a function of fraction thermal units, based on the data of $K_{C}$ inferred from the lysimeter with determination coefficients greater than 0.60 . The average values of $K_{C}$ for the bell pepper for the initial, middle, and final stages were $0.57,1.06$, and 0.80 , respectively. The $K_{C}$ values were similar to those proposed by Allen et al. [12] because the climatic conditions were similar in both studies, which were lower compared to the values of Shukla et al. [43], where the climatic conditions were similar but the rain and the contribution of the water table caused greater evaporation.

An increase in the bell pepper yield of $7.72 \%$ in 2019 and of $3.49 \%$ in 2020 was achieved compared to the yield established by the Ministry of the Environment and Rural and Marine Areas of the Spanish Government [6]. The $K_{C_{\text {lys }}}$ values and the FTU models for the bell pepper obtained in this study will help farmers to determine the water requirement and to improve the crop water efficiency in semiarid locations with conditions similar to those of this study.

Author Contributions: Conceptualization and methodology L.A..-D., J.M.M.-M., and C.B.-C.; validation and formal analysis, L.A.-D., J.M.M.-M., C.B.-C., M.S.-M., and J.G.-T;; investigation and resources, M.S.-M., H.E.J.-F., C.O.R.R., and J.G.-T.; writing - original draft L.Á.-D., J.M.M.-M., and C.B.-C.; writing - review and editing, M.S.-M., H.E.J.-F., C.O.R.R., and J.G.-T.; visualization and supervision, L.A.-D., J.M.M.-M., C.B.-C., M.S.-M., and J.G.-T.; project administration and funding acquisition, J.M.M.-M., and M.S.-M. All authors have read and agreed to the published version of the manuscript.

Funding: This research was financed by the Project of Research and Development, reference IDI20190146, titled "Development and implementation of a ferticontrol equipment by weighing lysimetry for use in intensive agriculture," in collaboration with the AGROSOLMEN, S.L. company, co-financed by the European Regional Development Fund (FEDER) through the Spanish Pluri-Regional Operational Programme 2014-2020.

Institutional Review Board Statement: Not applicable.

Informed Consent Statement: Not applicable.

Data Availability Statement: No new data were created or analyzed in this study. Data sharing is not applicable to this article.

Acknowledgments: The authors acknowledge CONACyT for the grant provided to L.A.-D. for her PhD studies.

Conflicts of Interest: The authors declare no conflict of interest.

References

1. Fernández-Pacheco, D.G.; Escarabajal-Henarejos, D.; Ruiz-Canales, A.; Conesa, J.; Molina-Martínez, J.M. A digital imageprocessing-based method for determining the crop coefficient of lettuce crops in the southeast of Spain. Biosyst. Eng. 2014, 117, 23-34. [CrossRef]

2. Escarabajal-Henarejos, D.; Molina-Martínez, J.M.; Fernández-Pacheco, D.G.; Cavas-Martínez, F.; García-Mateos, G. Digital photography applied to irrigation management of Little Gem lettuce. Agric. Water Manag. 2014, 151, 148-157. [CrossRef]

3. López-Urrea, R.; Montoro, A.; López-Fuster, P.; Fereres, E. Evapotranspiration and responses to irrigation of broccoli. Agric. Water Manag. 2009, 96, 1155-1161. [CrossRef]

4. Egbuikwem, P.N.; Obiechefu, G.C. Evaluation of Evapotranspiration Models for Waterleaf crop using Data from Lysimeter In Proceedings of the 2017 Spokane, Washington, DC, USA, 16-19 July 2017; American Society of Agricultural and Biological Engineers: St. Joseph Charter Township, MI, USA, 2017.

5. Yimam, A.Y.; Assefa, T.T.; Adane, N.F.; Tilahun, S.A.; Jha, M.K.; Reyes, M.R. Experimental Evaluation for the Impacts of Conservation Agriculture with Drip Irrigation on Crop Coefficient and Soil Properties in the Sub-Humid Ethiopian Highlands. Water 2020, 12, 947. [CrossRef]

6. Ministerio de Medio Ambiente y Medio Rural y Marino Estadísticas Agrarias. Available online: https://www.mapa.gob.es/es/ estadistica/temas/estadisticas-agrarias/ (accessed on 15 October 2020).

7. Nikolaou, G.; Neocleous, D.; Christou, A.; Kitta, E.; Katsoulas, N. Implementing Sustainable Irrigation in Water-Scarce Regions under the Impact of Climate Change. Agronomy 2020, 10, 1120. [CrossRef] 
8. González-Esquiva, J.M.; García-Mateos, G.; Escarabajal-Henarejos, D.; Hernández-Hernández, J.L.; Ruiz-Canales, A.; MolinaMartínez, J.M. A new model for water balance estimation on lettuce crops using effective diameter obtained with image analysis. Agric. Water Manag. 2017, 183, 116-122. [CrossRef]

9. Feltrin, R.M.; de Paiva, J.B.D.; de Paiva, E.M.C.D.; Beling, F.A. Lysimeter soil water balance evaluation for an experiment developed in the Southern Brazilian Atlantic Forest region. Hydrol. Process. 2011, 25, 2321-2328. [CrossRef]

10. Soldevilla-Martinez, M.; Quemada, M.; López-Urrea, R.; Muñoz-Carpena, R.; Lizaso, J.I. Soil water balance: Comparing two simulation models of different levels of complexity with lysimeter observations. Agric. Water Manag. 2014, 139, 53-63. [CrossRef]

11. Cunha, H.; Loureiro, D.; Sousa, G.; Covas, D.; Alegre, H. A comprehensive water balance methodology for collective irrigation systems. Agric. Water Manag. 2019, 223, 105660. [CrossRef]

12. Allen, R.G.; Pereira, L.S.; Raes, D.; Smith, M. Crop Evapotranspiration-Guidelines for Computing Crop Water Requirements-FAO Irrigation and Drainage Paper 56; FAO: Rome, Italy, 1998; Volume 300.

13. Sepaskhah, A.R.; Andam, M. Crop coefficient of sesame in a semiarid region of IR Iran. Agric Water Manag. 2001, 49, 51-63. [CrossRef]

14. Martínez-Cob, A. Use of thermal units to estimate com crop coefficients under semiarid climatic conditions. Irrig. Sci. 2008, 26, 335-345. [CrossRef]

15. Mateos, L.; González-Dugo, M.P.; Testi, L.; Villalobos, F.J. Monitoring evapotranspiration of irrigated crops using crop coefficients derived from time series of satellite images. I. Method validation. Agric. Water Manag. 2013, 125, 81-91. [CrossRef]

16. Kullberg, E.G.; DeJonge, K.C.; Chávez, J.L. Evaluation of thermal remote sensing indices to estimate crop evapotranspiration coefficients. Agric. Water Manag. 2017, 179, 64-73. [CrossRef]

17. Vaughan, P.J.; Trout, T.J.; Ayars, J.E. A processing method for weighing lysimeter data and comparison to micrometeorological ETo predictions. Agric. Water Manag. 2007, 88, 141-146. [CrossRef]

18. Evett, S.R.; Mazahrih, N.T.; Jitan, M.A.; Sawalha, M.H.; Colaizzi, P.D.; Ayars, J.E. A Weighing Lysimeter for Crop Water Use Determination in the Jordan Valley, Jordan. Trans. ASABE 2009, 52, 155-169. [CrossRef]

19. Bello, Z.A.; Van Rensburg, L.D. Development, calibration and testing of a low-cost small lysimeter for monitoring evaporation and transpiration: Development, calibration and testing of a low cost small lysimeter. Irrig. Drain. 2017, 66, 263-272. [CrossRef]

20. Benli, B.; Kodal, S.; Ilbeyi, A.; Ustun, H. Determination of evapotranspiration and basal crop coefficient of alfalfa with a weighing lysimeter. Agric. Water Manag. 2006, 81, 358-370. [CrossRef]

21. Anapalli, S.S.; Ahuja, L.R.; Gowda, P.H.; Ma, L.; Marek, G.; Evett, S.R.; Howell, T.A. Simulation of crop evapotranspiration and crop coefficients with data in weighing lysimeters. Agric. Water Manag. 2016, 177, 274-283. [CrossRef]

22. Wegehenkel, M.; Zhang, Y; Zenker, T.; Diestel, H. The use of lysimeter data for the test of two soil-water balance models: A case study. Z. Pflanzenernähr. Bodenk. 2008, 171, 762-776. [CrossRef]

23. Meissner, R.; Seeger, J.; Rupp, H.; Seyfarth, M.; Borg, H. Measurement of dew, fog, and rime with a high-precision gravitation lysimeter. I. Plant Nutr. Soil Sci. 2007, 170, 335-344. [CrossRef]

24. Schrader, F.; Durner, W.; Fank, J.; Gebler, S.; Pütz, T.; Hannes, M.; Wollschläger, U. Estimating Precipitation and Actual Evapotranspiration from Precision Lysimeter Measurements. Procedia Environ. Sci. 2013, 19, 543-552. [CrossRef]

25. Herbrich, M.; Gerke, H.H. Autocorrelation analysis of high resolution weighing lysimeter time series as a basis for determination of precipitation. J. Plant Nutr. Soil Sci. 2016, 179, 784-798. [CrossRef]

26. Hannes, M.; Wollschläger, U.; Schrader, F.; Durner, W.; Gebler, S.; Pütz, T.; Fank, J.; von Unold, G.; Vogel, H.-J. A comprehensive filtering scheme for high-resolution estimation of the water balance components from high-precision lysimeters. Hydrol. Earth Syst. Sci. 2015, 19, 3405-3418. [CrossRef]

27. Hoffman, M.; Schwartengräber, R.; Wessolek, G.; Peters, A. Comparison of simple rain gauge measurements with precision lysimeter data. Atmos. Res. 2016, 174-175, 120-123. [CrossRef]

28. Peters, A.; Nehls, T.; Schonsky, H.; Wessolek, G. Separating precipitation and evapotranspiration from noise-A new filter routine for high-resolution lysimeter data. Hydrol. Earth Syst. Sci. 2014, 18, 1189-1198. [CrossRef]

29. Young, M.H.; Wierenga, P.J.; Mancino, C.F. Large Weighing Lysimeters for Water Use and Deep Percolation Studies. Soil Sci. 1996, 161, 491-501. [CrossRef]

30. Ruiz-Peñalver, L.; Vera-Repullo, J.A.; Jiménez-Buendía, M.; Guzmán, I.; Molina-Martínez, J.M. Development of an innovative low cost weighing lysimeter for potted plants: Application in lysimetric stations. Agric. Water Manag. 2015, 151, 103-113. [CrossRef]

31. Nicolás-Cuevas, J.A.; Parras-Burgos, D.; Soler-Méndez, M.; Ruiz-Canales, A.; Molina-Martínez, J.M. Removable Weighing Lysimeter for Use in Horticultural Crops. Appl. Sci. 2020, 10, 4865. [CrossRef]

32. METER Group Smart Field Lysimeter. Available online: https://www.metergroup.com/environment/products/smart-fieldlysimeter/\%20or/ (accessed on 12 December 2020).

33. UGT-Team Lysimeter Technology. Available online: https://www.ugt-online.de/en/products/lysimeter-technology/ (accessed on 12 December 2020)

34. Aboukhaled, A.; Alfaro, A.; Smith, M. Lysimeters. In FAO Irrigation and Drainage Paper 39; Food and Agriculture Organization United Nations: Rome, Italy, 1982.

35. Soil Survey Staff. Soil Survey Manual; United State Department of Agriculture: Washington, DC, USA, 1951.

36. Ministerio de Agricultura, Pesca y Alimentación Guía Práctica de la Fertilización Racional de los Cultivos en España. Available online: https://www.mapa.gob.es/es/agricultura/publicaciones/Publicaciones-fertilizantes.aspx (accessed on 7 February 2019). 
37. Miranda, F.R.; Gondim, R.S.; Costa, C.A.G. Evapotranspiration and crop coefficients for tabasco pepper (Capsicum frutescens L.). Agric. Water Manag. 2006, 82, 237-246. [CrossRef]

38. Vidal, J.L. Efectos del Factor Térmico en el Desarrollo del Crecimiento Inicial de Pimiento (Capsicum annum L.) Cultivado en Campo; Universidad Nacional de Tucumán: Tucumán, Argentina, 2011.

39. Verma, S.P. Análisis Estadístico de Datos Composicionales; Universidad Autónoma de México: Ciudad de México, Mexico, 2016.

40. Wackerly, D.D.; Mendenhall, W.; Schaeaffer, R.L. Mathematical Statics with Applications, 7th ed.; Learning Cengage; Cengage Learning: Boston, MA, USA, 2010.

41. Willmott, C.; Ackleso, S.; Davis, O.; Feddema, J.; Klink, K.; Legate, D.; Rowe, A. Statistics for the Evaluation and Comparison of Models. J. Geophys. Res. Oceans 1985, 90, 8995-9005. [CrossRef]

42. Playán, E.; Mateos, L. Modernization and optimization of irrigation systems to increase water productivity. Agric. Water Manag 2006, 80, 100-116. [CrossRef]

43. Shukla, S.; Jaber, F.H.; Goswami, D.; Srivastava, S. Evapotranspiration losses for pepper under plastic mulch and shallow water table conditions. Irrig. Sci. 2013, 31, 523-536. [CrossRef]

44. Muniandy, J.M.; Yusop, Z.; Askari, M. Evaluation of reference evapotranspiration models and determination of crop coefficient for Momordica charantia and Capsicum annuum. Agric. Water Manag. 2016, 169, 77-89. [CrossRef]

45. López-Marín, J.; Angosto, J.L.; González, A. El cultivo de pimientos en invernadero y al aire libre. El caso del Campo de Cartagena. Bibl. Hortic. 2017, 1, 1-67. 


\subsubsection{Publicación 2}

Notificación de aceptación de la publicación

\begin{tabular}{ll} 
De: & Agronomy Editorial Office < agronomy@mdpi.com> \\
Recibido el: & 14/01/2021 \\
Para: & Julián González-Trinidad <jgonza@uaz.edu.mx> \\
& Laura Ávila-Dávila <laura_14avila@uaz.edu.mx>, \\
& Manuel Soler-Méndez <manuel.ia@agrosolmen.es>, \\
& Carlos Francisco Bautista-Capetillo <baucap@uaz.edu.mx>, \\
& Hugo Enrique Júnez-Ferreira <hejunez@uaz.edu.mx>, \\
CC: & Cruz Octavio Robles Rovelo <corr03@hotmail.com>, \\
& José Miguel Molina-Martínez <josem.molina@upct.es>, \\
& Agronomy Editorial Office <agronomy@mdpi.com> $>$ \\
Asunto: & [Agronomy] Manuscript ID: agronomy-1036484 - Accepted for Publication \\
\hline
\end{tabular}

Dear Dr. González-Trinidad,

We are pleased to inform you that the following paper has been officially

accepted for publication:

Manuscript ID: agronomy-1036484

Type of manuscript: Article

Title: A Compact Weighing Lysimeter to Estimate the Water Infiltration Rate in Agricultural Soils

Authors: Laura Ávila-Dávila, Manuel Soler-Méndez, Carlos Francisco Bautista-Capetillo, Julián González-Trinidad *, Hugo Enrique Júnez-Ferreira, Cruz Octavio Robles Rovelo, José Miguel Molina-Martínez *

Received: 27 November 2020

E-mails: laura_14avila@uaz.edu.mx, manuel.ia@agrosolmen.es,

baucap@uaz.edu.mx, jgonza@uaz.edu.mx, hejunez@uaz.edu.mx,corr03@hotmail.com, josem.molina@upct.es

https://susy.mdpi.com/user/manuscripts/review_info/0126b5d1ec7da43fccbc627a0451326b

We will now make the final preparations for publication, then return the manuscript to you for your approval. 
If, however, extensive English edits are required to your manuscript, we will need to return the paper requesting improvements throughout.

We encourage you to set up your profile at SciProfiles.com, MDPI's researcher network platform. Articles you publish with MDPI will be linked to your SciProfiles page, where colleagues and peers will be able to see all of your publications, citations, as well as your other academic contributions.

We also invite you to contribute to Encyclopedia (https:/encyclopedia.pub), a scholarly platform providing accurate information about the latest research results. You can adapt parts of your paper to provide valuable reference information for others in the field.

Kind regards,

Martina Buljovcic

Section Managing Editor

E-mail: buljovcic@mdpi.com

MDPI

Agronomy Editorial Office

MDPI Novi Sad Branch Office

Bulevar Oslobođenja 83

21000 Novi Sad

Serbia

Tel. +381213001449

E-Mail: agronomy@mdpi.com

http://www.mdpi.com/journal/agronomy/ 


\title{
A Compact Weighing Lysimeter to Estimate the Water Infiltration Rate in Agricultural Soils
}

\author{
Laura Ávila-Dávila ${ }^{1,2}{ }^{(}$, Manuel Soler-Méndez ${ }^{2}$, Carlos Francisco Bautista-Capetillo ${ }^{1}{ }^{(0)}$,

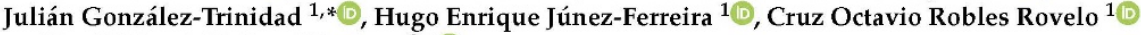 \\ and José Miguel Molina-Martínez ${ }^{2, *}$ (D) \\ 1 Research Group Doctorado en Ciencias de la Ingeniería, Autonomous University of Zacatecas, Campus UAZ \\ Siglo XXI, Zacatecas 98160, Mexico; laura_14avila@uaz.edu.mx (L.Á.-D.); baucap@uaz.edu.mx (C.F.B.-C.); \\ hejunez@uaz.edu.mx (H.E.J.-F.); corr03@hotmail.com (C.O.R.R.) \\ 2 Research Group Ingeniería Agromótica y del Mar, Technical Universy of Cartagena, Campus Alfonso XIII, \\ ETSIA, 30203 Cartagena, Spain; manuel.ia@agrosolmen.es \\ * Correspondence: jgonza@uaz.edu.mx (J.G.-T.); josem.molina@upct.es (J.M.M.-M.); \\ Tel.: + 34-968-32-5929 (J.M.M.-M.)
}

\section{check for
updates}

Citation: Ávila-Dávila, Lr; Soler-Méndez, M.; Bautista-Capetillo, C.F.; González-Trinidad, J.; Júnez-Ferreira, H.E.; Robles Rovelo, C.O.; Molina-Martínez, J.M. A Compact Weighing Lysimeter to Fstimate the Water Infiltration Rate in Agricultural Soils. Agronomy 2021, 11, 180. https://doi.org/10.3390/ agronomy 11010180

Received: 27 November 2020 Accepted: 14 January 2021 Published: 18 January 2021

Publisher's Note: MDPI stays neutral with regard to jurisdictional claims in published maps and institutional affiliations.

\section{(c) (i)}

Copyright: 2021 by the authors Licensee MDPI, Basel, Switzerland. This article is an open access article distributed under the terms and conditions of the Creative Commons Attribution (CC BY) license (https:// creativecommons.org/licenses/by/ $4.0 /$ )

\begin{abstract}
Infiltration estimation is made by tests such as concentric cylinders, which are prone to errors, such as the lateral movement under the ring. Several possibilities have been developed over the last decades to compensate these errors, which are based on physical, electronic, and mathematical principles. In this research, two approaches are proposed to measure the water infiltration rate in a silty loam soil by means of the mass values of a lysimeter weighing under rainfall conditions and different moisture contents. Based on the fact that with the lysimeter it is possible to determine acting soil flows very precisely, then with the help of mass conservation and assuming a downward vertical movement, 12 rain events were analyzed. In addition, it was possible to monitor the behavior of soil moisture and to establish the content at field capacity from the values of the weighing lysimeter, from which both approach are based. The infiltration rate of these events showed a variable rate at the beginning of the rainfall until reaching a maximum, to descend to a stable or basic rate. This basic infiltration rate was $1.49 \pm 0.36 \mathrm{~mm} / \mathrm{h}$, and this is because soils with fine textures have reported low infiltration capacity. Four empirical or semi-empirical models of infiltration were calibrated with the values obtained with our approaches, showing a better fit with the Horton's model.
\end{abstract}

Keywords: soil moisture; water balance; vertical movement; drainage; rainfall; water mass

\section{Introduction}

To understand the behavior of the hydrological cycle in the soil remains a challenge for science, specifically the movement of water through the soil and its capacity to retain it. To estimate the water exchange between a well-defined portion of soil and other physical systems to which water is transferred through evaporation, transpiration, percolation or drainage, several methods as gravimetric, tensiometry, humidity sensing, and lysimetry have been proposed in the specialized literature [1-3].

Infiltration is the hydrological process that describes the entry of water into a soil, and the amount of water that enters the soil in a given time represents the infiltration rate [4,5]. It is variable in space and time, due to hydrodynamic and physical soil properties, flow conditions, and cultivation practices, among others [6-9]. As water enters the soil profile through the forces of adhesion, the interaction of water and soil particles, and gravity, it fills the pores of the soil, defining a wet front as it passes through the soil profile. If the soil has a good structure and large pores, its infiltration rate will be high (as in the case of coarse-textured soils) and it will reach a deeper wet front compared to a fine-textured soil whose pores are smaller. It is assumed that if the soil is at its lower limit of initial 
moisture content, a higher infiltration rate would be observed [10-14]. This is not true for hydrophobic or water-repellent soils caused by high organic matter and clay content [15].

The first model to describe the movement of water into a saturated soil profile is the well-known Darcy's law, which states that the flow through the soil column is linearly proportional to the cross-section and the hydraulic gradient [4]. Years later, a correction was made to apply it to unsaturated soils with the Darcy-Buckingham's law, which states that if the liquid pressure increases, the proportionality constant will increase, because the air-filled spaces will decrease [7]. Richards [16] proposed another model for unsaturated soil as a result of Darcy's law and the concept of the capillary potential of liquids in a porous media. There are simplified models based on soil physical properties were developed for unidirectional infiltration. One of them is the well-known Kostiakov's model developed from experimental observations related to water volume moving on soil versus time, but it has no physical meaning with the soil [4]. Similarly, experimental techniques - such Horton's model-have been proposed which arise from simplifications made to continuity equation to estimate infiltration rate [17].

The single and double ring infiltrometers allow to measure the soil infiltration rate in field tests, due to their simplicity and easy operation in one dimension $[6,18,19]$. These instruments assume that the infiltration is vertical, and although they are easy to use, the test requires sufficient time to obtain a better estimation of the infiltration $[20,21]$. Such assumption it is not true in most cases, then a better understanding of this phenomenon occurs when more precise instruments are used to measure the infiltration rate taking into account the water movement in other directions, one of which is the weighing lysimeter, which is based on water balance flows. This allows for the estimation of water and solute flows in the entire profile of saturated or unsaturated soils [22-24].

The weighing lysimeter can be used in experiments under different conditions (crop variety, ground composition, atmospheric conditions, and irrigation events) and to extrapolate the behavior to great land extensions and optimize the hydric resources $[25,26]$. These devices have been used to quantify precipitation, condensation, and determine crop evapotranspiration [27-33]. Moreover, they could be used to characterize the contribution of groundwater and determine the percentage of leachate for a crop in real-time [34] or the upward water flow of a groundwater table [35]. Studies of water movement into the soil with the use of the lysimeter and different sensors of water content or matric potential were conducted [36-38]. To better understand the behavior of infiltration and surface runoff when there is heavy rain, it is necessary to further investigate the lysimeters calibration. See Haselow et al. [27] for information to understand this phenomenon.

Usually, lysimeters have been used for research purposes at experimental facilities of agricultural centers sponsored by governments. Although over time, the dimensions of weighing lysimeter have been declining, they are still of robust architecture and relatively expensive, especially thinking of furthering their use among farmers. The main objective of this work was to determine the infiltration rate of a silty loam soil in function of the soil field capacity content, using the mass data of a compact weighing lysimeter. The results were used to calibrate the Kostiakov's models, the modified Kostiakov's model, the Horton and the Philip models by using the data collected with the lysimeter.

\section{Materials and Methods}

2.1. Study Area

Field measurements were performed on Las Tiesas farm with one hectare of extension in Albacete, Spain $\left(39^{\circ} 3^{\prime} 31^{\prime \prime} \mathrm{N}, 2^{\circ} 6^{\prime} 04^{\prime \prime} \mathrm{W}\right)$ at an elevation of 695 m.a.s.l. (Figure 1) during 2017. Albacete is characterized by a semi-arid climate, with an average annual rainfall of $384 \mathrm{~mm}$ and high temperatures in summer (average maximum value of $40^{\circ} \mathrm{C}$, minimum of $-6^{\circ} \mathrm{C}$, and an average of $30^{\circ} \mathrm{C}$ ). 


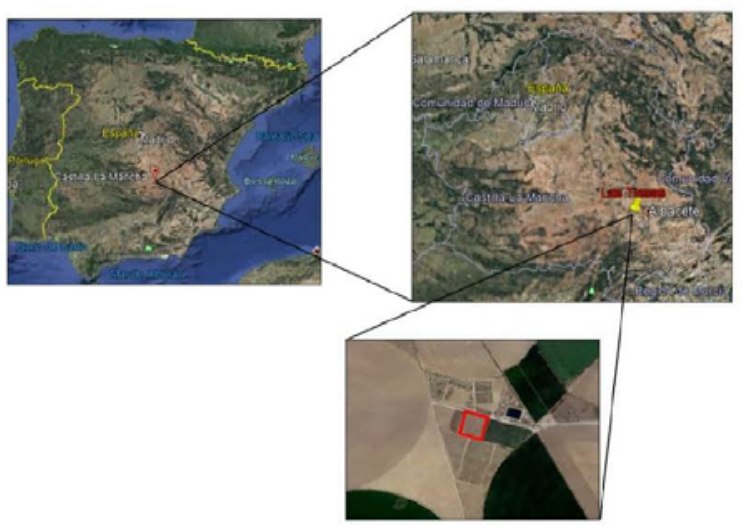

Figure 1. Location of the Study Area [39].

The soil texture was classified as silt loam, based on the soil percentages obtained with Bouyoucos hydrometer method and the USDA texture triangle figure [40]. Percentages of soil granulometry are $27.1 \%$ sand, $51.5 \%$ silt, and $21.4 \%$ clay; an organic matter of $2.77 \%$, and a bulk density of $1.38 \mathrm{~g} / \mathrm{cm}^{3}$. The hydrodynamic characteristics of the field capacity and permanent wilting point were obtained by the membrane and pressure plate apparatus, which resulted in $0.35 \mathrm{~cm}^{3} / \mathrm{cm}^{3}$ and $0.16 \mathrm{~cm}^{3} / \mathrm{cm}^{3}$, respectively. The initial soil moisture content on February 8 were the experiment started was inferred knowing the bulk density and total volume with $0.23 \mathrm{~cm}^{3} / \mathrm{cm}^{3}$.

\subsection{Materials}

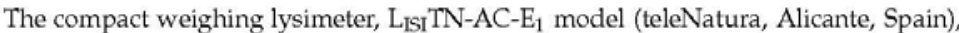
consists of two vessels. The upper vessel called soil vessel has dimensions of $0.56 \mathrm{~m} \times 0.96 \mathrm{~m}$ and $0.35 \mathrm{~m}$ depth in the center, which stores a volume of the reconstituted soil from the plot trying to keep the same conditions from where it is extracted, with a resolution of $20 \mathrm{~g}$ or $0.033 \mathrm{~mm}$ (Figure 2). The soil is isolated of its environment in such a manner that the lateral and capillary rise underground flows are zero [26], so that the water balance terms can be accurately obtained.

The second vessel is located below the previous one. It is called drainage vessel and serves to store the excess of water drained by the soil profile with a resolution of $1 \mathrm{~g}$ (or $0.002 \mathrm{~mm}$ ). The soil vessel has a hole in the lower central part that allows the water flows to an electrovalve, then goes to a funnel and finally reaches the drainage vessel, where it will be stored and weighed (Figure $2 \mathrm{c}$ ). The drainage vessel was configured to store only $3000 \mathrm{~g}$ of water, so when this increase is reported, the electrovalve located under it is activated and starts emptying it, while this occurs the electrovalve of the soil vessel is closed to avoid losses of water quantification. Once the emptying process has been completed, the electrovalves return to their initial state, opening the soil vessel one and closing the drainage vessel one, allowing the water flow from the soil vessel to the drainage vessel.

The mass variations of both vessels are detected by load cells, four for the soil vessel and one for the drainage vessel. These load cells send an electrical signal to the datalogger (CR300 model, Campbell Scientific, Logan, UT, USA), according to the deformation suffered by the object that they are supporting. This electrical signal is converted to a mass value by the same datalogger. According to the Spanish norm UNE 500520 [41], the mass data of the two vessels was sampled every second and the average value of $60 \mathrm{~s}$ (one minute) was registered as an instantaneous value. This guarantees the maximum quality and representativeness of the data obtained. For further details of the compact weighing lysimeter see the work of Nicolás-Cuevas et al. [42]. 


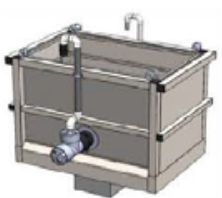

(a)

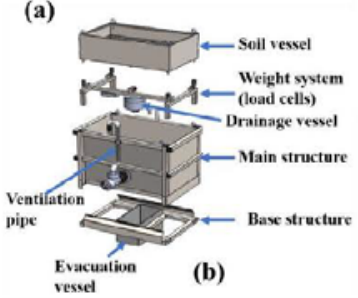

(c)

Figure 2. Compact weighing lysimeter (a) full view, (b) exploded view and (c) water movement through the vessels.

\subsection{Flow Calculation}

The lysimeter contains an isolated soil profile, so that is possible to determine the water inputs and outputs in the soil based on the mass data records, through the mass balance equation, shown in Equation (1):

$$
\frac{d S}{d t}=\frac{d P}{d t}+\frac{d I R}{d t}-\frac{d D}{d t}-\frac{d E T}{d t}-\frac{d S r}{d t}
$$

where $\pm \frac{d S}{d t}$ is the change of soil water storage $(\mathrm{g}), \frac{d P}{d t}$ is the amount of water by precipitation $(\mathrm{g}), \frac{d I R}{d t}$ is the amount of water by irrigation $(\mathrm{g}), \frac{d D}{d t}$ is the amount of water drainage beyond the root zone $(\mathrm{g}), \frac{d E T}{d t}$ is the amount of water by the crop evapotranspiration $(\mathrm{g})$, and $\frac{d S r}{d t}$ is the surface runoff $(\mathrm{g})$ along interval of time [6].

In this work, only the rainfall events were analyzed to know the rate of entry of water into the soil. The rainfall has a longer duration of application and therefore gives a better accuracy of the results. So, variables as irrigation and evapotranspiration were not considered, except in determining $\theta_{F C}$, because they cannot occur when there is rain $[32,43]$, so we reformulate Equation (1) as Equation (2):

$$
\frac{d S}{d t}=\frac{d P}{d t}-\frac{d D}{d t}-\frac{d S r}{d t}
$$

Surface runoff is neglected because it was not observed here. The only known variables are the accumulated masses of the soil vessel $(S)$ and the drainage vessel $(D)$, so the flow is determined by the increase in the mass $(m)$ of the vessel over time [32], as the Equation (3):

$$
\frac{d S}{d t} \text { or } \frac{d D}{d t}=\frac{m_{t_{i+1}}-m_{t_{i}}}{t_{i+1}-t_{i}}
$$

where $\frac{d D}{d t}$ is the flow of drainage vessel over time, $\frac{d S}{d t}$ is the flow of the soil vessel over time, $t_{i}$ is the time in the instant $i$ and $t_{i+1}$ is the time in the instant $i+1$.

Rainfall is calculated based on literature reports $[27,32,43,44]$, so that the lysimeter must satisfy the condition $\frac{d D}{d t}+\frac{d S}{d t}>0$. Therefore, the following Equation (4) was used:

$$
\frac{d P}{d t}=\frac{d D}{d t}+\frac{d S}{d t}
$$




\subsection{Calculation of the Infiltration Rate of Water into the Soil}

The water infiltration has a downward vertical behavior in the lysimeter [26], and conditions of free drainage [7]. When the soil moisture was below field capacity water content, the increments of soil water stored in the soil vessel represent the soil infiltration rate $\frac{d l}{d t}$ without any drainage component $\frac{d D}{d t}$. The infiltration rate was then represented with the following Equation (5) which was called Approach 1 [45]:

$$
\frac{d I}{d t}=\frac{d S}{d t}
$$

However, once the soil reached the water content at field capacity, it is no longer able to store more water and thus, the incoming water will be drained [45]. In that way, the sum of soil water storage and drainage represents the water infiltration rate of the soil profile. The infiltration rate model is represented as the Equation (6) which was called Approach 2:

$$
\frac{d I}{d t}=\frac{d S}{d t}+\frac{d D}{d t}
$$

Both approaches are valid if the water content at soil saturation is not reached due to the rainfall rate not overpassing the soil basic infiltration capacity. In this experiment the soil never overpass the saturation capacity, therefore there was no surface runoff. This complies with the principle of mass conservation and continuity equation.

The resulting flows and infiltration rate are representing the area of the lysimeter $\left(0.54 \mathrm{~m}^{2}\right)$, so with the assumption that $1 \mathrm{~L}$ of water is equal to $1 \mathrm{~kg}$ of water mass and that $1 \mathrm{~mm}$ is equal to $1 \mathrm{~L} / \mathrm{m}^{2}$ [27], $1 \mathrm{~kg} / \mathrm{min}$ of lysimeter is equal to $1.85 \mathrm{~kg} / \mathrm{min}$ or $1.85 \mathrm{~mm} / \mathrm{min}$ of $1 \mathrm{~m}^{2}$ of surface.

The irrigation events were not analyzed because their time and volume of application were small, so they do not allow a visualization of the infiltration capacity curve. The infiltration rate was constant throughout the two or three hours that the irrigations lasted.

\subsection{Estimation of Soil Moisture Content}

The soil profile water content inside the soil vessel was determined by the gravimetric method, since the mass of the wet soil profile was known at any time. Once obtained, the gravimetric water content was converted to volumetric $\left(\theta_{v}\right)$ as in Equation (7):

$$
\theta_{v}=\left(\frac{m_{i}-m_{d r y}}{m_{d r y}}\right) \times \rho_{b}
$$

where $\theta_{v}$ is the volumetric water content is of the soil at instant $i\left(\mathrm{~m}^{3} / \mathrm{m}^{3}\right), \rho_{b}$ is the bulk density of the soil, $m_{i}$ is the wet mass of the soil inside the soil vessel at instant $i$ ( $\mathrm{g}$ or $\mathrm{kg}$ ) and $m_{d r y}$ is the dry mass of the soil inside the soil vessel. This last value was determined from the apparent density of the soil and the total volume of the soil vessel $\left(0.161 \mathrm{~m}^{3}\right)$ [13]. The moisture content at field capacity $\left(\theta_{F C}\right)$ was established two or three days after the rain events, when the soil stopped draining and the soil vessel value was constant, which indicates that the soil is already capable of retaining water [4]. No runoff was observed in any of the rain events. Evapotranspiration $\left(\frac{d E T}{d t}\right)$ was calculated because it affects the redistribution of soil moisture, which was obtained using the mass balance method (Equation (1)), remembering that evapotranspiration and rain cannot occur at the same time. The mass at instant $i$ represents soil-water aggregates, and the mass of the vessel structure is considered zero as it was tared to zero before inserting the soil profile into the soil vessel.

\subsection{Validation}

A total of 12 rain events of 2017 were analyzed from 8 February till 30 May, which were (i) February 13, (ii) 18 February, (iii) 19 February, (iv) 24 February, (v) 13 March, (vi) 14 
March, (vii) 27 April, (viii) 28 April, (ix) 29 April, (x) 30 April, (xi) 10 May and (xii) 30 May. Approach 2 could be used only for events (v) to $(x)$ while drainage occurred.

\subsection{Model Calibrations}

The results of the water infiltration rate $\left(\frac{d I}{d t}\right)$ into the soil were used to calibrate empirical and physics infiltration models from the previously mentioned authors.

One of the most used models to represent the infiltration rate is the Kostiakov's model, which can be generally represented as Equation (8):

$$
\frac{d I}{d t}=a b t^{b-1}
$$

where $t$ is the infiltration time and $a$ and $b$ are empirical constants $[4,6,46-48]$. This equation has a boundary condition: $\lim _{t \rightarrow \infty} \frac{d I}{d t}=0[49,50]$. All empirical parameters of all infiltration equations were determined by an iterative fit of the experimental data obtained with our approaches and selected using the best root mean square error (RMSE) in the fitting [46].

Parameter $b$ of Kostiakov's equation is limited to being greater than zero and less than one. Therefore, the initial value of the infiltration rate tends to be infinite and has a basic rate to zero in an infinite time. In long irrigation events, Lewis [47] observed that the infiltration rate tends to a constant value before the end of the irrigation. So, in these long events, Kostiakov's equation usually overestimates the infiltration [51,52]. Therefore, a constant was added to this equation to represent the basic infiltration rate, which resulted in the modified Kostiakov's equation:

$$
\frac{d I}{d t}=a b t^{b-1}+c
$$

where $c$ is an empirical constant, which represents the basic infiltration rate $[6,53,54]$. This equation also is known as the Kostiakov-Lewis or Mezencev model. Its boundary condition is: $\lim \frac{d I}{d t}=$ constant value $=c[49]$.

Philip's equation for the homogeneous porous medium infiltration rate is a simplification of Richard's model. The first term of the equation establishes the absorption of water into the soil and the second term represents a truncated series of small flooding times $(A)$, as the Equation (10):

$$
\frac{d I}{d t}=\frac{1}{2} S t^{-1 / 2}+A
$$

where $S$ is the sorptivity $[50,53]$ and $\lim _{t \rightarrow \infty} \frac{d I}{d t}=A$, where $A$ is a constant value [49].

Horton establishes an equation of infiltration capacity based on the behavior of nature that follows a law of inverse exponential. The infiltration rate decreases to a constant value, with an exponential decrease of change, and proportional to the infiltrated water volume as follows:

$$
\frac{d I}{d t}=V_{f}+\left(V_{0}-V_{f}\right) e^{-\beta \times t}
$$

where $V_{f}$ is the final infiltration rate or basic rate, $V_{0}$ is the initial infiltration rate, and $\beta$ is an empirical constant of change from the initial rate to the basic rate [17]. The boundary condition is $\lim _{t \rightarrow \infty} \frac{d I}{d t} \geq 0$ [50].

When comparing the four models, it can be seen that the parameters of Kostiakov's model have no physical meaning and for longer times of water infiltration, it loses its representativeness and accuracy of the rate of water infiltration into the soil. For $t=0$, the infiltration rate remains undetermined. In the modified Kostiakov's model, a term was added to represent the basic rate observed at long times of water infiltration. In the case of the Horton model, the initial infiltration rate is defined by a finite value and the model describes the behavior of the soil infiltration capacity curve. Finally, Philip described the rate of infiltration into the soil surface when there is a small amount of ponding, so its 
terms do have physical significance for the behavior of the soil when water is allowed to enter.

The statistics used to evaluate the accuracy of the estimation of the water infiltration rate into the soil using the weighing lysimeter and using the above-mentioned models were: the standard deviation (s), the root mean square error (RMSE), and the coefficient of determination $\left(\mathrm{R}^{2}\right)[55]$.

\section{Results and Discussion}

Figure 3 shows the mass curves of the two vessels of the lysimeter in the analyzed time interval. The initial data reported by the soil vessel indicates the mass of the soil particles plus the water content. Consequently, the mass variations reported after this initial point indicate the variations of the water content within the soil. In the curve of the soil vessel, the inputs were the rain and the irrigation and they can be differentiated by the type of increase they present. Irrigation has a very vertiginous increase in a short time, while the rain has a discontinuous growth in time, on the other hand, the descents can represent the evapotranspiration losses, drainage losses or both.

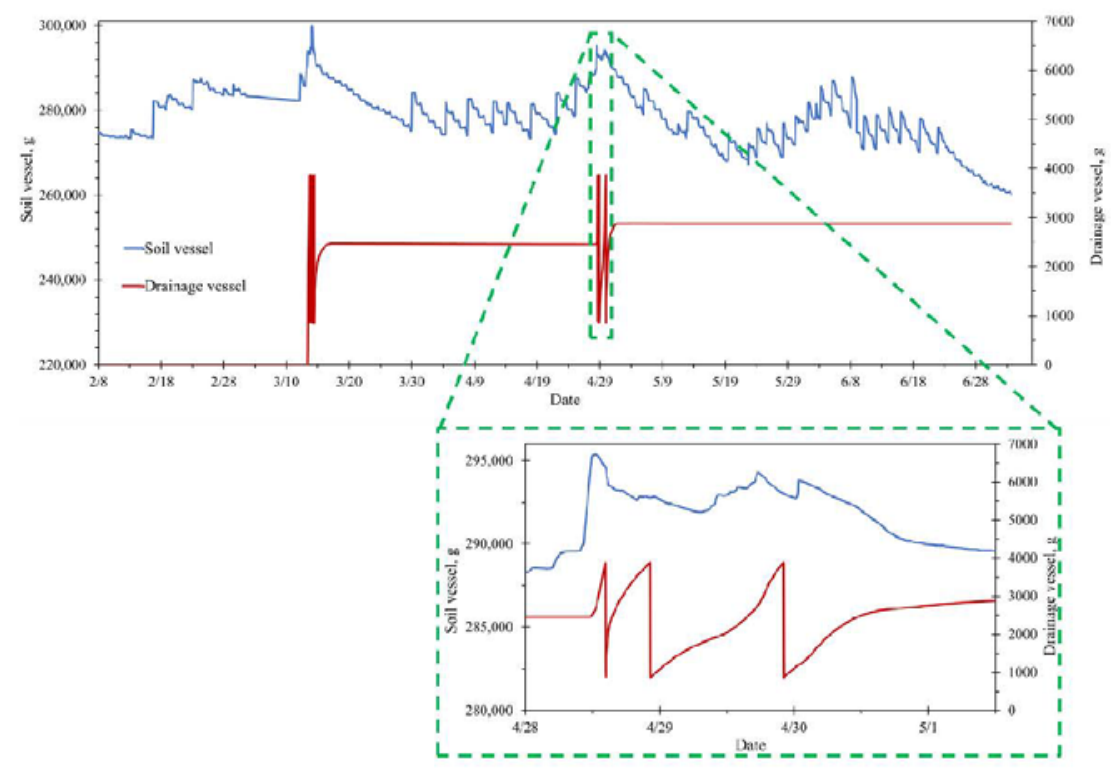

Figure 3. Mass variations of the two lysimeter vessels during 2017.

In the case of the data from the drainage vessel (red curve), the rise of the curve represents that the drainage has started and the sudden descents show us the emptying of the vessel. As shown in the Figure 3, only two drainage events were registered by the vessel on 13-14 March and 28-30 April. The drainage vessel was programmed to open its valve at $3000 \mathrm{~g}$ of storage and discharge (to avoid damaging the load cell). Therefore, the drainage vessel curve in the Figure 3 shows accumulated masses less than $4000 \mathrm{~g}$. The emptying is almost instantaneous, it takes less than $3 \mathrm{~min}$ and, as mentioned, no drop is lost. The March event caused more drainage than the April event, in less time of rain. This indicates that the rain intensity of the March event was higher than in the April event, ass more water fell in less time. The seven rainfall events between February and March contributed to the first drainage event occurred in 14 March. Meanwhile, the five rainfall events occurred between April and May caused the second drainage event in 28-30 April. 


\subsection{Estimation of Rain Inflow}

The rainy days total of 12 were analyzed and were determined with the Equation (4), representing 12 events. The Table 1 shows the rainfall recorded by day in the weighing lysimeter and those observed in a rain gauge installed near the weighing lysimeter at $800 \mathrm{~m}$. The rain calculated with the lysimeter for the first analysis day concerning the Instituto Técnico Agronómico Provincial (ITAP, Spanish acronym) station has a difference of $0.05 \mathrm{~mm}$. The differences found can be explained by the space behavior of the rainfall variability in a region, which has a greater impact in semi-arid zones such as the study zone [56]. There is an absolute average difference of $1.59 \mathrm{~mm}$ between the two devices.

Table 1. Rainfall intensity data of the analyzed days recorded by two different devices.

\begin{tabular}{ccc}
\hline & \multicolumn{2}{c}{ Rain Registered per Day } \\
\cline { 2 - 3 } Date & Weighing Lysimeter & Weather Station ITAP \\
\cline { 2 - 3 } & $(\mathbf{m m})$ & $(\mathbf{m m})$ \\
\hline $02 / 13$ & 3.55 & 3.60 \\
$02 / 18$ & 3.16 & 3.30 \\
$02 / 19$ & 2.76 & 2.90 \\
$02 / 24$ & 1.30 & 1.00 \\
$03 / 13$ & 36.77 & 26.70 \\
$03 / 14$ & 28.53 & 22.30 \\
$04 / 27$ & 7.04 & 6.40 \\
$04 / 28$ & 7.06 & 5.90 \\
$04 / 29$ & 8.16 & 8.30 \\
$04 / 30$ & 2.44 & 1.6 \\
$05 / 10$ & 1.01 & 1.10 \\
$05 / 30$ & 2.11 & 1.60 \\
\hline
\end{tabular}

\subsection{Estimation of Soil Moisture Content}

The soil moisture content was obtained using the gravimetric method (Equation (7)). Two drainage events were caused with the rain events of 13-14 March, 28-30 April so only with these events was possible to determine the soil field capacity $\left(\theta_{F C}\right)$ based on its definition. The Figure 4 shows the rain behavior and its relation to the moisture content of the soil, which shows that when rain is falling, there is a mass increase, and when the soil reaches the field capacity and the rain continues, there is drainage; when there is a fall in the humidity curve, drainage increases.

By plotting the water content curve with that of the drainage vessel (red curve) it was possible to determine the moment when the moisture content at field capacity of the soil $\left(\theta_{F C}\right)$ was reached. In both months, the $\theta_{F C}$ was established when the drainage vessel curve became constant, indicating that the soil had stopped draining and there was no variation in the weighing lysimeter.

A horizontal line was drawn on the water content at the instant the mass drainage vessel was not increased, indicating that the $\theta_{F C}$ was reached and was established two days after the beginning of the rain in $0.33 \mathrm{~cm}^{3} / \mathrm{cm}^{3}$.

Since the $\theta_{F C}$ was established days after the rain events, evapotranspiration $\left(\frac{d E T}{d t}\right)$ was presents, so it was calculated as indicated by $[27,32,43]$ in their work. Table 2 has the water balance of the days involved in the calculation of the $\theta_{F C} \cdot \frac{d E T}{d t}$ is a variable that subtracts moisture from the soil surface, this variable for the first days of each event only represents $0 \%$ of water losses and rainfall; it increases as the rain decreases over the days, so that in the second day $\frac{d E T}{d t}$ represents $15.47 \%$ and $10.32 \%$ of water losses and $18.96 \%$ and $7.83 \%$ of rainfall. The total $\frac{d E T}{d t}$ losses for all days of each event used for the $\theta_{F C}$ calculation represent $11.04 \%$ for the first (Figure $4 \mathrm{a}$ ) and $24.37 \%$ for the second (Figure $4 \mathrm{~b}$ ) $\frac{d E T}{d t}$ represents $8.28 \%$ of the rain of the first event (Figure $4 \mathrm{a}$ ) and $35.38 \%$ of the second event (Figure 4b). 

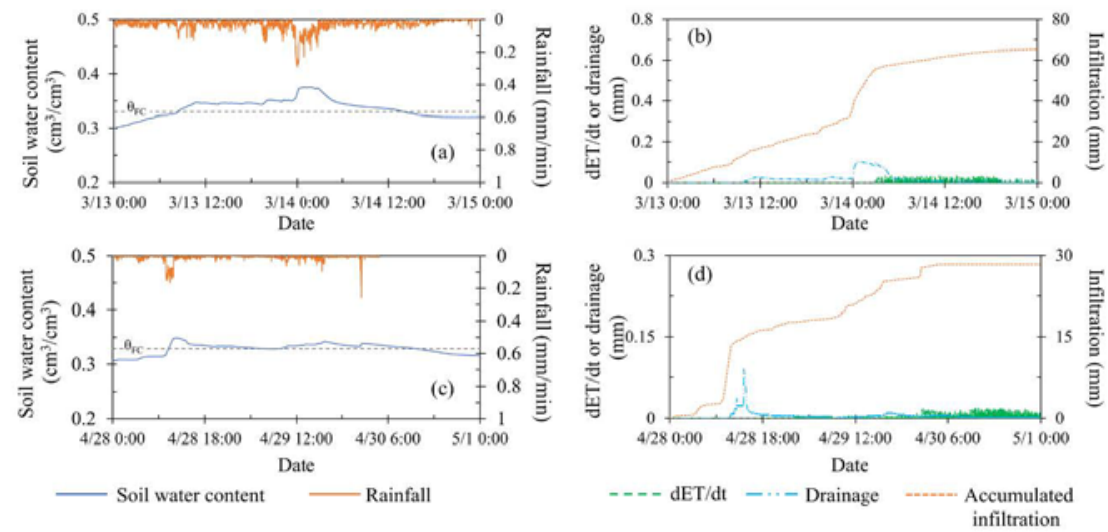

Figure 4. Soil water content and hydrologic variables obtained with the weighing lysimeter, (a) Soil water content and rainfall of March; (b) evapotranspiration ( $\left.\frac{d E T}{d t}\right)$ and infiltration of March; (c) soil water content and rainfall of April; (d) $\frac{d E T}{d t}$ and infiltration of Abril.

Table 2. Water balance of the days involved in determining the $\theta_{F C}$.

\begin{tabular}{ccccc}
\hline Date & $\frac{d I R}{d t}, \mathrm{~mm}$ & $\frac{d P}{d t}, \mathrm{~mm}$ & $\frac{d D}{d t}, \mathrm{~mm}$ & $\frac{d E T}{d t}, \mathrm{~mm}$ \\
\hline $03 / 13$ & 0.00 & 36.77 & 14.04 & 0.00 \\
$03 / 14$ & 0.00 & 28.53 & 29.57 & 5.41 \\
$04 / 28$ & 10.62 & 5.90 & 8.65 & 0.00 \\
$04 / 29$ & 0.00 & 8.30 & 5.65 & 0.65 \\
$04 / 30$ & 0.00 & 1.60 & 3.05 & 4.94 \\
\hline
\end{tabular}

The Table 3 reports the moisture content at field capacity values obtained and some that report the literature for a silty loam soil. The values with less standard deviation with the result of the weighing lysimeter were the value obtained in the laboratory in this investigation and the maximum value reported by Assi et al. [57] with $0.014 \mathrm{~cm}^{3} / \mathrm{cm}^{3}$. These are followed by the values reported by FAO [58] in graphs and tables based on soil texture to obtain the hydrodynamic characteristics of the soil ( $\theta_{\text {saturation, }} \theta_{F C}$ and $\left.\theta_{\text {wilting point }}\right)$ with $0.02 \mathrm{~cm}^{3} / \mathrm{cm}^{3}$, which shows a good agreement with results obtained in laboratory and lysimeter.

Table 3. Comparison of field water content value obtained in the soil with literature values.

\begin{tabular}{ccc}
\hline$\theta_{F C}, \mathrm{~cm}^{3} / \mathrm{cm}^{3}$ & Method of Estimation and Authors \\
\hline 0.33 & Weighing Lysimeter & \\
0.35 & Laboratory & {$[58]$} \\
$0.22-0.36$ & FAO & {$[57]$} \\
$0.30-0.31$ & Pedostructure & {$[59]$} \\
0.244 & Gravimetric & \\
\hline
\end{tabular}

\subsection{Water Infiltration Rate}

Seven rains were analyzed with the Approach 1 . The soil was below field capacity so that the positive variations reported soil vessel indicated the water infiltration rate $(13,18$, 19 and 24 February, 27 April and 10 and 30 May). According to USDA [60] and Evanylo and McGuinn [61] the best way to determine the rate or speed of infiltration is when the soil is close to field capacity; four rain events had drainage due to soil was above field capacity; in these rains, approach 2 was applied (13-14 March and 28, 29 and 30 April). 
Figure 5 shows the 11 scatter soil water infiltration rate diagrams of the rain 12 events analyzed, most of the graphs have a duration of less than $24 \mathrm{~h}$. The soil shows a very similar behavior in all the graphs, it starts with lower rates until it reached a maximum peak and then decreases to a stable or basic rate. The basic infiltration rate was obtained considering the eleven rainfall events in $1.49 \mathrm{~mm} / \mathrm{h}$ with a $0.36 \mathrm{~mm} / \mathrm{h}$ standard deviation. According to USDA [60], this rate occurs when the soil is almost saturated and it does not decrease or increase as more water is added.
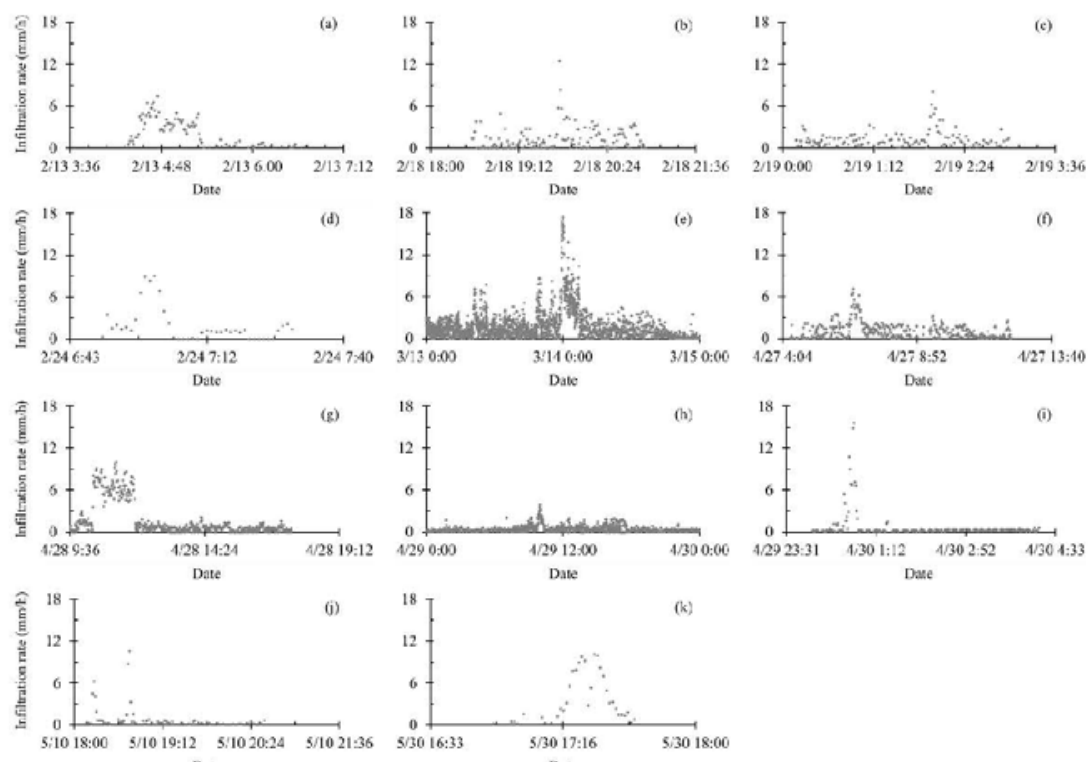

Date

Date

Figure 5. Soil water infiltration rate scatter diagrams, (a) 13 February; (b) 18 February; (c) 19 February; (d) 24 February; (e) 13-14 March; (f) 27 April; (g) 28 April; (h) 29 April; (i) 30 April; (j) 10 May; (k) 30 May.

The variability of the standard deviation could be due the experimental error. The infiltration rate or speed modifications reaching a $23 \%$, could probably be due the flow paths alterations at forcing the water to pass through an orifice. Further research is needed in order to minimize this variability, i.e, a number of orifices placed in the horizontal plane in the soil vessel bottom could represent a variability of the measured infiltration rate/speed minimizing the measurement errors. Nevertheless, our methodology represents an approach to estimate the infiltration rate and could be compared with other methodologies.

The variability of the initial infiltration rate may be due to rainfall intensity and initial moisture content $[62,63]$. The initial moisture contents of the seven infiltration rate scatter diagrams below field capacity ranged from 0.23 to $0.30 \mathrm{~cm}^{3} / \mathrm{cm}^{3}$ and the other four scatter diagrams were 0.30 to $0.33 \mathrm{~cm}^{3} / \mathrm{cm}^{3}$.

The basic infiltration found in both approaches (summarized in the Table 4) were low compared to the values reported in the literature. However, the values found in this investigation coincide with those reported by Li et al. [64] for homogeneous wetting and their value was obtained with an initial soil water content of $0.034 \mathrm{~m}^{3} / \mathrm{m}^{3}$, which was air-dried and placed in a $50 \mathrm{~cm}$ column. The lower value obtained in Li et al. [64] was with a slightly water-repellent silty loam soil (this is achieved by adding octadecylamine to the soil). 
Table 4. Values of the infiltration rate of a silty loam soil.

\begin{tabular}{ccc}
\hline Basic Infiltration Rate, $\mathrm{mm} / \mathrm{h}$ & \multicolumn{2}{c}{ Method and Authors } \\
\hline $1.13-1.85$ & Weighing lysimeter & \\
$10.00-14.00$ & Concentric cylinders & {$[65]$} \\
$2.00-3.00$ & Homogeneous wetting & {$[64]$} \\
$0.15-0.30$ & Slightly water-repellent soil & {$[64]$} \\
$5.00-8.00$ & Concentric cylinders & {$[6]$} \\
\hline
\end{tabular}

The basic infiltration rate obtained with the weighing lysimeter, was lower compared with the research works of Maldonado [65] and Ali [6] considering the same soil type. Some factors that could have caused the low value of the basic infiltration rate are organic matter content, vegetative cover, and the crop root $[6,63]$. Nevertheless, further investigation is needed to corroborate it.

The results obtained with the two proposed approaches are simple and representative of the current capacity of infiltration of the soil, where roots have been growing within the soil profile, fertilizers have been added, and the profile has been compacted by the same force exerted by rainwater and irrigation. All of this is not reflected in the values seen in Table 4

\subsection{Model Calibrations}

The 11 infiltration curves were calibrated with the four models described above, from its highest point. In Figure 6, only the four models with longer rainfall duration are presented. The model that showed the best fit was Horton's, with a RMSE of $0.89 \pm 0.40$ and $R^{2}$ of $0.65 \pm 0.30$, followed by Kostiakov with a RMSE of $1.06 \pm 0.40$ and $R^{2}$ of $0.63 \pm 0.23$, modified Kostiakov with a RMSE of $1.03 \pm 0.43$ and $R^{2}$ of $0.62 \pm 0.22$, and Philip with a RMSE of $1.30 \pm 0.42$ and $R^{2}$ of $0.48 \pm 0.17$. The only model that established a lower initial infiltration rate was Horton.

The models establish the value for the basic infiltration rate in some of its variables in the equation. In the case of Kostiakov, it does not have an established value. Modified Kostiakov established the basic infiltration at $0.14 \pm 0.14 \mathrm{~mm} / \mathrm{h}$ (in its constant c) [53,54], Philip at $0.16 \pm 0.11 \mathrm{~mm} / \mathrm{h}$ (in A), and Horton at $0.38 \pm 0.34 \mathrm{~mm} / \mathrm{h}$ (in $V_{f}$ ) $[17,50]$ The models calibration showed a behavior as indicated by their boundary limits, as the infiltration rate tends to constant values close to zero when time tends to infinity $[49,50]$.
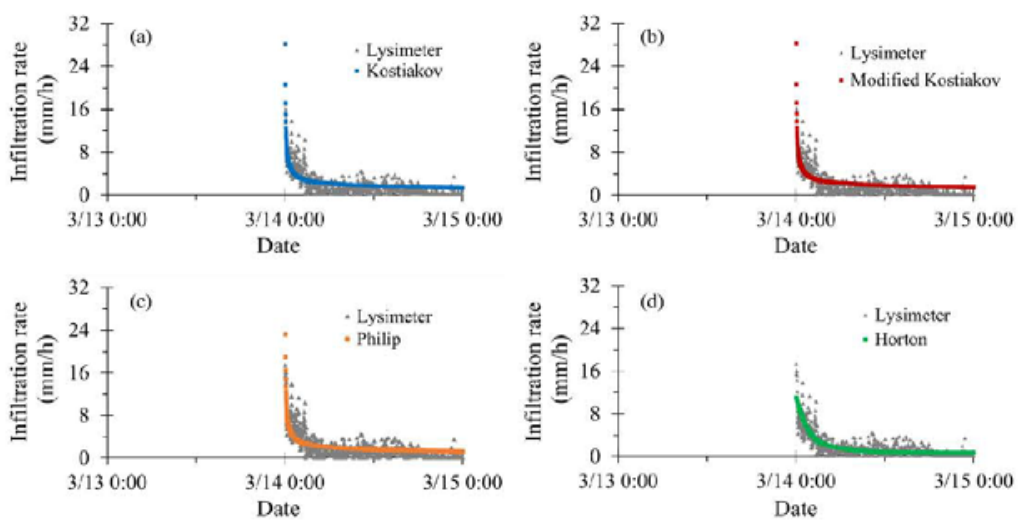

Figure 6. Adjustment of the infiltration models to the values of March 13-14, (a) Kostiakov model; (b) Modified Kostiakov model; (c) Philip model; (d) Horton model. 
In the research works of Rodríguez-Vásquez et al., [66] and Mirzaee et al., [67] measured infiltration rate with double ring infiltrometer apparatus for silt loam and clayey soil, their parameter values obtained for the different infiltration models are shown in the Table 5. In the first work, the water contents were between $24.3 \%$ and $31.1 \%$, while in the second article this was not mentioned, and our range was of $0.23-0.33 \mathrm{~cm}^{3} / \mathrm{cm}^{3}$.

Table 5. Parameters values of infiltration models for silt loam soil.

\begin{tabular}{ccccc}
\hline \multicolumn{1}{c}{ Model } & & Lysimeter & Rodríguez-Vásquez et al. [66] & Mirzaee et al. [67] \\
\hline \multirow{2}{*}{ Kostiakov } & a & 47.70 & 66.46 & 1.00 \\
& b & 0.62 & 1.12 & 3.11 \\
\hline \multirow{2}{*}{ Modified } & a & 48.42 & & 3.23 \\
Kostiakov & $\mathrm{b}$ & 0.50 & - & 0.84 \\
& $\mathrm{c}$ & 0.29 & 13.6 & 1.00 \\
\hline \multirow{2}{*}{ Philip } & $\mathrm{S}$ & 20.83 & 7.93 & 31.3 \\
& $\mathrm{~A}$ & 0.30 & - & 1.00 \\
\hline \multirow{3}{*}{ Horton } & $V_{O}$ & 14.44 & & 58.8 \\
& $V_{f}$ & 0.75 & & 34.8 \\
& $\beta$ & 0.28 & & 1.00 \\
\hline
\end{tabular}

The fit parameters of Kostiakov model in this investigation compared with the Rodríguez-Vásquez et al., [66], have a difference of $39 \%$ for the parameter a, while for the parameter $b$ is of $20 \%$. In the Philip model, there is a difference of $35 \%$ for $\mathrm{S}$ parameter and the value of A parameter of Rodríguez-Vásquez et al., [66] is 25 times higher than ours. This difference probably is due to that error of estimation is greater in the concentric cylinders.

There is a significant difference with respect to Mirzaee et al., [67] work in the four models varying from 68 to $245 \%$. because their reported values are of a soil profile with siltyloam and clayey horizons, which the infiltration capacity is very low due to characteristics of the clays.

\section{Conclusions}

In this research, the mass values of a compact weighing lysimeter were used to measure soil moisture behavior and the water infiltration rate of a soil silt loam under unsteady rainfall conditions, using two approaches, the first part when the soil is below and the second when the soil is above field capacity. The results showed that with the two approaches it is possible to establish the soil infiltration curve and for short and long application times in real conditions. The soil is subject to a variable and faster infiltration rate at the beginning, but it reaches a basic or stable infiltration as the soil fills its pores.

These approaches are simple but satisfy the conservation of mass and continuity equation for incomprehensible flows. Although they are used for non-saturation conditions, they can be used for saturation conditions by adding the surface runoff variable that would subtract incoming flow to the soil.

The use of the weighing lysimeter with these approaches allowed to characterize the infiltration rate of a homogeneous and non-homogeneous soil at depths where agriculture regularly takes place. However, the limitation is that it is not possible to monitor the progress of the wet front, so further research is necessary in order to have greater measurement instruments (i.e., soil moisture sensors) to characterize the water movement in the soil and the forces acting within it (i.e., soil water potential sensor).

Author Contributions: Conceptualization and methodology, L.A.-D.; experiment development J.M.M.-M. and M.S.-M.; data analysis, L.A.-D., J.M.M.-M., and M.S.-M.; calibration and fitting of the mathematical models, C.F.B.-C., J.G.-T., and H.E.J.-F.; investigation, L.A..-D., C.F.B.-C., J.G.-T., C.O.R.R., and J.M.M.-M.; writing —original draft preparation, L.A.-D.; writing-review and editing, C.F.B.-C., J.G.-T., H.E.J.-F., and C.O.R.R.; funding acquisition M.S.-M. and J.M.M.-M. All authors have read and agreed to the published version of the manuscript. 
Funding: Project of Research and Development with reference IDI-20190146 titled "Development and implementation of a ferticontrol equipment by weighing lysimetry for use in intensive agriculture", in collaboration with the AGROSOLMEN, S.L. company, co-financed by the European Regional Development Fund (FEDER) through the Spanish Pluri-regional Operational Programme 2014-2020. Institutional Review Board Statement: Not applicable.

Informed Consent Statement: Not applicable.

Data Availability Statement: No new data were created or analyzed in this study. Data sharing is not applicable to this article.

Acknowledgments: The authors express their gratitude to the Mexican National Council for Science and Technology (CONACYT) for financing the scholarship of the doctoral student.

Conflicts of Interest: The authors declare no conflict of interest. The funders had no role in the design of the study; in the collection, analyses, or interpretation of data; in the writing of the manuscript, or in the decision to publish the results.

\section{References}

1. Struthers, I.; Hinz, C.; Sivapalan, M.; Deutschmann, G.; Beese, F.; Meissner, R. Modelling the water balance of a free-draining lysimeter using the downward approach. Hydrol. Process. 2003, 17, 2151-2169. [CrossRef]

2. Wegehenkel, M.; Zhang, Y; Zenker, T.; Diestel, H.; Zhang, Y. The use of lysimeter data for the test of two soil-water balance models: A case study. J. Plant Nutr. Soil Sci. 2008, 171, 762-776. [CrossRef]

3. Feltrin, R.M.; de Paiva, J.B.D.; de Paiva, E.M.C.D.; Beling, F.A. Lysimeter soil water balance evaluation for an experiment developed in the Southern Brazilian Atlantic Forest region. Hydrol. Process. 2011, 25, 2321-2328. [CrossRef]

4. Kirkham, M. Water Movement in Saturated Soil. Princ. Soil Plant. Water Relat. 2014, 87-101. [CrossRef]

5. Mendes, W.R.; de Araújo, F.M.U.; Dutta, R.; Heeren, D.M. Fuzzy control system for variable rate irrigation using remote sensing. Expert Syst. Appl. 2019, 124, 13-24. [CrossRef]

6. Ali, M.H. Fundamentals of Irrigation and On-farm Water Management: Volume 1; Springer Science and Business Media LLC: Berlin/Heidelberg, Germany, 2010.

7. Herrada, M.A.; Gutiérrez-Martin, A.; Montanero, J.M. Modeling infiltration rates in a saturated/unsaturated soil under the free draining condition. J. Hydrol. 2014, 515, 10-15. [CrossRef]

8. Mattar, M.A.; Alazba, A.A.; El-Abedin, T.K. Forecasting furrow irrigation infiltration using artificial neural networks. Agric. Water Manag. 2015, 148, 63-71. [CrossRef]

9. Yuan, J.; Feng, W.; Jang, X.; Wang, J. Saline-alkali migration in soda saline soil based on sub-soiling technology. Desalin. Water Treat. 2019, 149, 352-362. [CrossRef]

10. Duchaufour, P. Manual de Edafología; Masson S.A.: Barcelona, Spain, 1987; ISBN 9788431104191

11. Plaster, E.J. Soil Science \& Management; Editorial Paraninfo: Madrid, Spain, 2000; ISBN 84-283-2643-6.

12. Wang, K.; Yang, X.; Liu, X.; Liu, C. A simple analytical infiltration model for short-duration rainfall. J. Hydrol. 2017, 555, 141-154. [CrossRef]

13. Villalobos, FJ.; Mateos, L.; Orgaz, F.; Fereres, E. Fitotecria Bases y Tecnologías de La Producción Agrícola; Mundi-Pren: Madrid, Spain, 2002; ISBN 9788484760498

14. Martín-Benito, J.M. El Riego Por Aspersión; Universidad de Castilla La Mancha: Ciudad Real, Spain, 1991.

15. Harper, R.; McKissock, I.; Gilkes, R.; Carter, D.; Blackwell, P. A multivariate framework for interpreting the effects of soil properties, soil management and landuse on water repellency. J. Hydrol. 2000, 232, 371-383. [CrossRef]

16. Richards, L.A. Capillary Conduction of Liquids Through Porous Mediumus. Physics 1931, 1, 318-333. [CrossRef]

17. Horton, R.E. An Approach Toward a Physical Interpretation of Infiltration-Capacity 1. Soil Sci. Soc. Am. J. 1941, 5, 399-417. [CrossRef]

18. Mahmood, S.; Latif, M. A Simple Procedure for Simulating Surge Infiltration Using First-Surge Infiltrometer Data. Irrig. Drain. 2005, 54, 407-416. [CrossRef]

19. Teófilo-Salvador, E.; Morales-Reyes, G.P. Propuesta del modelo físico del infiltrómetro de cilindros concéntricos rediseñado multifuncional (ICCRM). Tecnol. Cienc. Agua 2018, 9, 103-131. [CrossRef]

20. Arriaga, F.J.; Kornecki, T.S.; Balkcom, K.S.; Raper, R.L. A method for automating data collection from a double-ring infiltrometer under falling head conditions. Soil Use Manag. 2009, 26, 61-67. [CrossRef]

21. Fatehnia, M.; Paran, S.; Kish, S.; Tawfiq, K. Automating double ring infiltrometer with an Arduino microcontroller. Geoderme 2016, 262, 133-139. [CrossRef]

22. Groh, J.; Vanderborght, J.; Pütz, T.; Vereecken, H. How to Control the Lysimeter Bottom Boundary to Investigate the Effect of Climate Change on Soil Processes? Vadose Zone J. 2016, 15, 1-15. [CrossRef]

23. Lepore, B.J.; Norman, J.M.; Lowery, B; Brye, K.R. Soil Compaction above Long-Term Lysimeter Installations. Soil Sci. Soc. Am. J 2011, 75, 30-34. [CrossRef] 
24. Masarik, K.C.; Norman, J.M.; Mason, R.E.; Baker, J.M. Improvements to Measuring Water Flux in the Vadose Zone. J. Environ. Qual. 2004, 33, 1152-1158. [CrossRef]

25. Jiménez-Buendía, M.; Ruiz-Peñalver, L.; Vera-Repullo, J.; Intrigliolo-Molina, D.; Molina-Martínez, J. Development and assessment of a network of water meters and rain gauges for determining the water balance. New SCADA monitoring software. Agric. Water Manag. 2015, 151, 93-102. [CrossRef]

26. Ruiz-Peñalver, L.; Vera-Repullo, J.; Jiménez-Buendía, M.; Guzman, I.; Molina-Martínez, J. Development of an innovative low cost weighing lysimeter for potted plants: Application in lysimetric stations. Agric. Water Manag. 2015, 151, 103-113. [CrossRef]

27. Haselow, L.; Meissner, R.; Rupp, H.; Miegel, K. Evaluation of precipitation measurements methods under field conditions during a summer season: A comparison of the standard rain gauge with a weighable lysimeter and a piezoelectric precipitation sensor. J. Hydrol. 2019, 575, 537-543. [CrossRef]

28. Meissner, R.; Seeger, J.; Rupp, H.; Seyfarth, M.; Borg, H. Measurement of dew, fog, and rime with a high-precision gravitation lysimeter. J. Plant. Nutr. Soil Sci. 2007, 170, 335-344. [CrossRef]

29. Schrader, F.; Durner, W.; Fank, J.; Gebler, S.; Pütz, T.; Hannes, M.; Wollschläger, U. Estimating Precipitation and Actual Evapotranspiration from Precision Lysimeter Measurements. Procedia Env. Sci. 2013, 19, 543-552. [CrossRef]

30. Valtanen, M.; Sillanpăă, N.; Setălă, H. A large-scale lysimeter study of stormwater biofiltration under cold climatic conditions. Ecol. Eng. 2017, 100, 89-98. [CrossRef]

31. Marek, G.; Gowda, P.; Marek, T.; Auvermann, B.; Evett, S.; Colaizzi, P.; Brauer, D. Estimating preseason irrigation losses by characterizing evaporation of effective precipitation under bare soil conditions using large weighing lysimeters. Agric. Water Manag. 2016, 169, 115-128. [CrossRef]

32. Hannes, M.; Wollschläger, U.; Schrader, F.; Durner, W.; Gebler, S.; Pütz, T.; Fank, J.; Von Unold, G.; Vogel, H.-J. High-resolution estimation of the water balance components from high-precision lysimeters. Hydrol. Earth Syst. Sci. Discuss. 2015, 12, 569-608. [CrossRef]

33. López-Urrea, R.; Montoro, A.; Mañas, F.; López-Fuster, P.; Fereres, E. Evapotranspiration and crop coefficients from lysimeter measurements of mature 'Tempranillo' wine grapes. Agric. Water Manag. 2012, 112, 13-20. [CrossRef]

34. Luo, Y.; Sophocleous, M. Seasonal groundwater contribution to crop-water use assessed with lysimeter observations and model simulations. J. Hydrol. 2010, 389, 325-335. [CrossRef]

35. Kelleners, T.J.; Soppe, R.; Ayars, J.E.; Simunek, J.; Skaggs, T.H. Inverse Analysis of Upward Water Flow in a Groundwater Table Lysimeter. Vadose Zone J. 2005, 4, 558-572. [CrossRef]

36. Dijkema, J.; Koonce, J.; Shillito, R.; Ghezzehei, T.; Berli, M.; van der Ploeg, M.; Van Genuchten, M. Water Distribution in an Arid Zone Soil: Numerical Analysis of Data from a Large Weighing Lysimeter. Vadose Zone J. 2017, 17, 170035. [CrossRef]

37. Germann, P.; Prasuhn, V. Viscous Flow Approach to Rapid Infiltration and Drainage in a Weighing Lysimeter. Vadose Zone I. 2017, 17, 170020. [CrossRef]

38. Schwaerzel, K.; Bohl, H.P. An easily installable groundwater lysimeter to determine water balance components and hydraulic properties of peat soils. Hydrol. Earth Syst. Sci. 2003, 7, 23-32. [CrossRef]

39. Google Earth. Available online: https://earth.google.com/web/ (accessed on 14 March 2020).

40. Conklin, H.E. Soil Survey Manual. J. Farm. Eøon. 1952, 34, 145. [CrossRef]

41. Automatic Weather Station Network. Criteria for the Localization of Sites and Installation of Sensor. Adquisition Characteristics and Sampling. In UNE 500520-2002; Spanish Standardization (UNE, Spanish Acronyms). Elaborated by the Technical Committee AEN/CTN GET5 Meteorological Records Whose Secretariat Is Provided by AENOR-PUERTOS DEL ESTADO; Spanish Association for Standardization and Certification (AENOR, Spanish Acronyms): Madrid, Spain, 2002.

42. Nicolás-Cuevas, J.A.; Parras-Burgos, D.; Soler-Méndez, M.; Ruiz-Canales, A.; Molina-Martínez, J.M. Removable Weighing Lysimeter for Use in Horticultural Crops. Appl. Sci. 2020, 10, 4865. [CrossRef]

43. Peters, A.; Nehls, T; Schonsky, H.; Wessolek, G. Separating precipitation and evapotranspiration from noise-A new filter routine for high-resolution lysimeter data. Hydrol. Earth Syst. Sci. 2014, 18, 1189-1198. [CrossRef]

44. Gebler, S.; Hendricks-Franssen, H.-J.; Putz, T.; Post, H.; Schmidt, M.; Vereecken, H. Actual evapotranspiration and precipitation measured by lysimeters: A comparison with eddy covariance and tipping bucket. Hydrol. Earth Syst. Sci. 2015, 19, 2145-2161 [CrossRef]

45. Wang, N.; Chu, X. Revised Horton model for event and continuous simulations of infiltration. J. Hydrol. 2020, 589 , 125215. [CrossRef]

46. Hartley, D.M. Interpretation of Kostiakov Infiltration Parameters for Borders. J. Irrig. Drain. Eng. 1992, 118, 156-165. [CrossRef]

47. Lewis, M.R. The rate of infiltration of water in irrigation-practice. Trans. Am. Geophys. Union 1937, 18, 361-368. [CrossRef]

48. Fok, Y. Derivation of Lewis-Kostiakov Intake Equation. J. Irrig. Drain. Eng. 1986, 112, 164-171. [CrossRef]

49. Haverkamp, R.; Kutilek, M.; Parlange, J.Y.; Rendon, L.; Krejca, M. Infiltration under Ponded Conditions: 2. Infiltration Equations Tested for Parameters Time-Dependece and Predictive Use1. Soil Sci. 1988, 145, 317-329. [CrossRef]

50. Philip, J.R. The Theory of Infiltration: 4. Sorptivity and Algebraic Infiltration Equation. Soil Sci. 1957, 84, 257-264. [CrossRef]

51. Smerdon, E.T;; Blair, A.W.; Reddell, D.L. Infiltration from Irrigation Advance Data. I: Theory. J. Irrig. Drain. Eng. $1988,114,4-17$. [CrossRef]

52. Haghiabi, A.H.; Abedi-Koupai, J.; Heidarpour, M.; Mohammadzadeh-Habili, J. A New Method for Estimating the Parameters of Kostikov and Modified Kostiakov Infiltration Equations. World Appl. Sci. J. 2011, 15, 129-135. 
53. Strelkoff, T.S.; Clemmens, A.J.; Bautista, E. Field Properties in Surface Irrigation Management and Design. J. Irrig. Drain. Eng. 2009, 135, 525-536. [CrossRef]

54. Furman, A.; Warrick, A.W.; Zerihun, D.; Sanchez, C.A. Modified Kostiakov Infiltration Function: Accounting for Initial and Boundary Conditions. J. Irrig. Drain. Eng. 2006, 132, 587-596. [CrossRef]

55. Wackerly, D.D.; Mendenhall, W.; Schaeaffer, R.L. Mathematical Statics with Applications, 7th ed.; Ceneage Learning: Boston, MA, USA, 2010.

56. Belmonte, A.M.C.; García, J.S.; García, M.J.L. The effect of observation timescales on the characterisation of extreme Mediterranean precipitation. Adv. Geosci. 2010, 26, 61-64. [CrossRef]

57. Assi, A.; Blake, J.; Mohtar, R.H.; Braudeau, E. Soil aggregates structure-based approach for quantifying the field capacity, permanent wilting point and available water capacity. Irrig. Sci. 2019, 37, 511-522. [CrossRef]

58. Allen, R.G.; Pereira, L.S.; Raes, D.; Smith, M. Crop Evapotranspiration-Guidelines for Computing Crop Water Requirements; FAO Irrigation and Drainage Paper 56; FAO Rome: Roma, Italy, 1998; Volume 300, p. d05109.

59. Porta-Casanellas, J.; Lopez-Acevedo, R.M. Agenda de Campo de Suelos. Información de Suelos Para La Agricultura y El Medio Ambiente; Ediciones Mundi-Prensa: Madrid, Spain, 2005.

60. USDA (United State Department of Agricultura); NRCS (Natural Resources Conservation Service); ARS (Agricultural Research Service); SQI (Soil Quality Institute). Soil Quality Test Kit Guide; U.S. Department of Agriculture: Washington, DC, USA, 2001.

61. Evanylo, G.; McGuinn, R. Agricultural Management Practices and Soil Quality; Virginia Polytechnic Institute and State University, College of Agriculture and Life Sciences: Blacksburg, VA, USA, 2000; Volume 5.

62. Cui, Z.; Wu, G.-L.; Huang, Z.; Liu, Y. Fine roots determine soil infiltration potential than soil water content in semi-arid grassland soils. J. Hydrol. 2019, 578, 124023. [CrossRef]

63. Liu, Y.; Cui, Z.; Huang, Z; López-Vicente, M.; Wu, G.-L. Influence of soil moisture and plant roots on the soil infiltration capacity at different stages in arid grasslands of China. Catena 2019, 182, 104147. [CrossRef]

64. Li, Y.; Ren, X.; Hill, R.; Malone, R.; Zhao, Y.; Ying, Z.; Malonew, R. Characteristics of Water Infiltration in Layered Water-Repellent Soils. Pedosphere 2018, 28, 775-792. [CrossRef]

65. Maldonado, T. Manual de Riego Parcelario. Available online: http://www.fao.org/tempref/GI/Reserved/FTP_FaoRlc/old/ prior/recnat/pdf/MR_cap1.PDF (accessed on 30 January 2020).

66. Rodríguez-Vásquez, A.F.; Aristizábal-Castillo, A.M.; Camacho-Tamayo, J.H. Variabilidad Espacial de los Modelos de Infiltración de Philip y Kostiakov en un Suelo Ândico. Eng. Agric. 2008, 12, 64-75. [CrossRef]

67. Mirzaee, S.; Zolfaghari, A.A.; Gorji, M.; Dyck, M.; Dashtaki, S.G. Evaluation of infiltration models with different numbers of fitting parameters in different soil texture classes. Arch. Agron. Soil Sci. 2013, 60, 681-693. [CrossRef] 


\section{5. Índices de impacto de las revistas}

\subsubsection{Sustainability Journal}
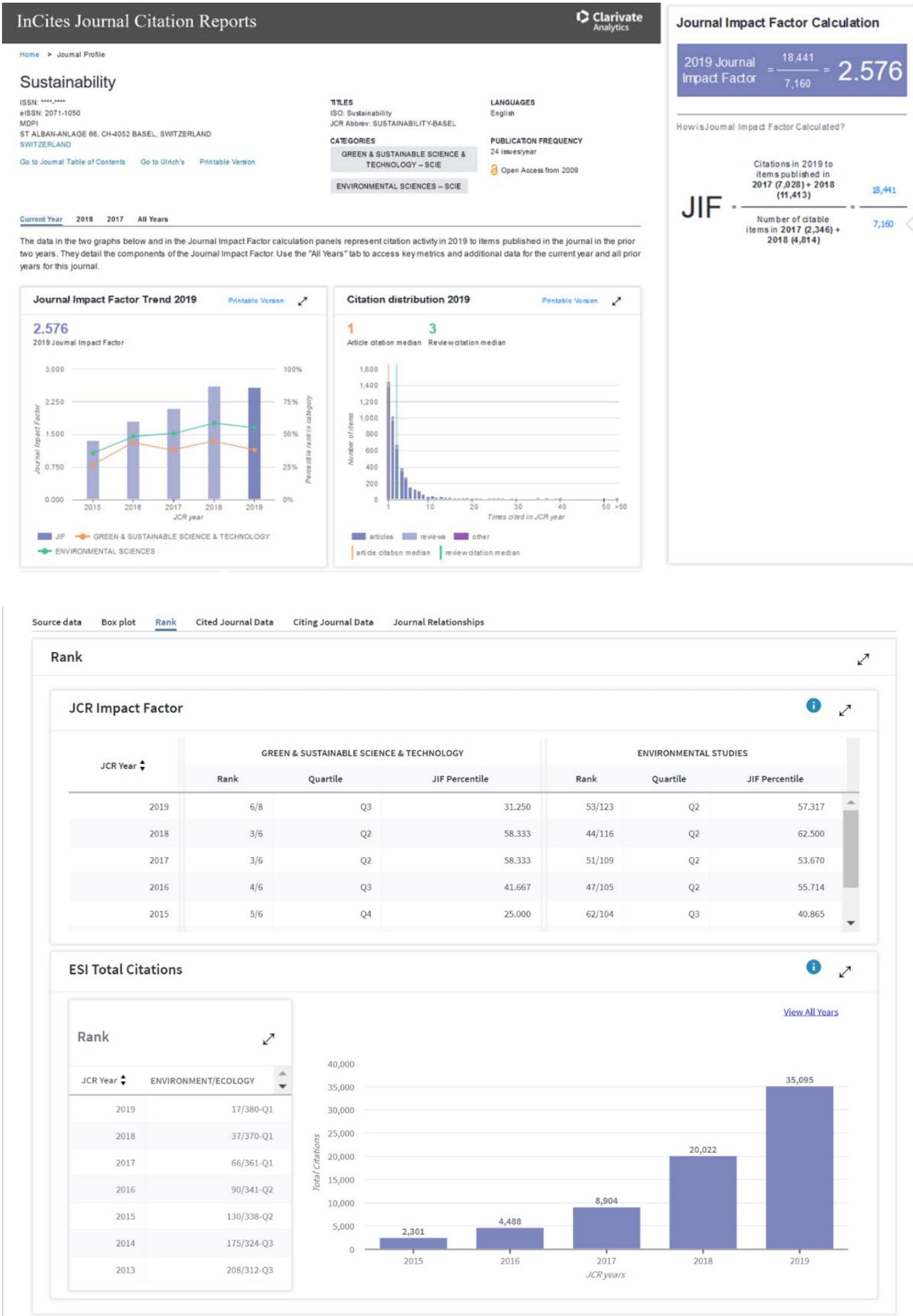


\subsubsection{Agronomy Journal}

InCites Journal Citation Reports

Home > Journal Profile

Agronomy-Basel

ISSN: 2073-4395

elSSN: 2073-439

MDPI

ST ALBAN-ANLAGE 66, CH-4052 BASEL, SWITZERLAND

SWITZERLAND

Go to Journal Table of Contents Go to Ulrich's Printable Version

\begin{tabular}{ll}
$\begin{array}{l}\text { TITLES } \\
\text { ISO: Agronomy-Basel }\end{array}$ & LANGUAGES \\
JCR Abbrev: AGRONOMY-BASEL & English \\
CATEGORIES & \\
\hline AGRONOMY-SCIE & PUBLICATION FREQUENCY \\
\hline PLANT SCIENCES-SCIE & 12 issues/year \\
\hline
\end{tabular}

Current Year $2018 \quad 2017 \quad$ All Years

The data in the two graphs below and in the Journal Impact Factor calculation panels represent citation activity in 2019 to items published in the journal in the prior two years. They detail the components of the Journal Impact Factor. Use the "All Years" tab to access key metrics and additional data for the current year and all prior years for this journal.

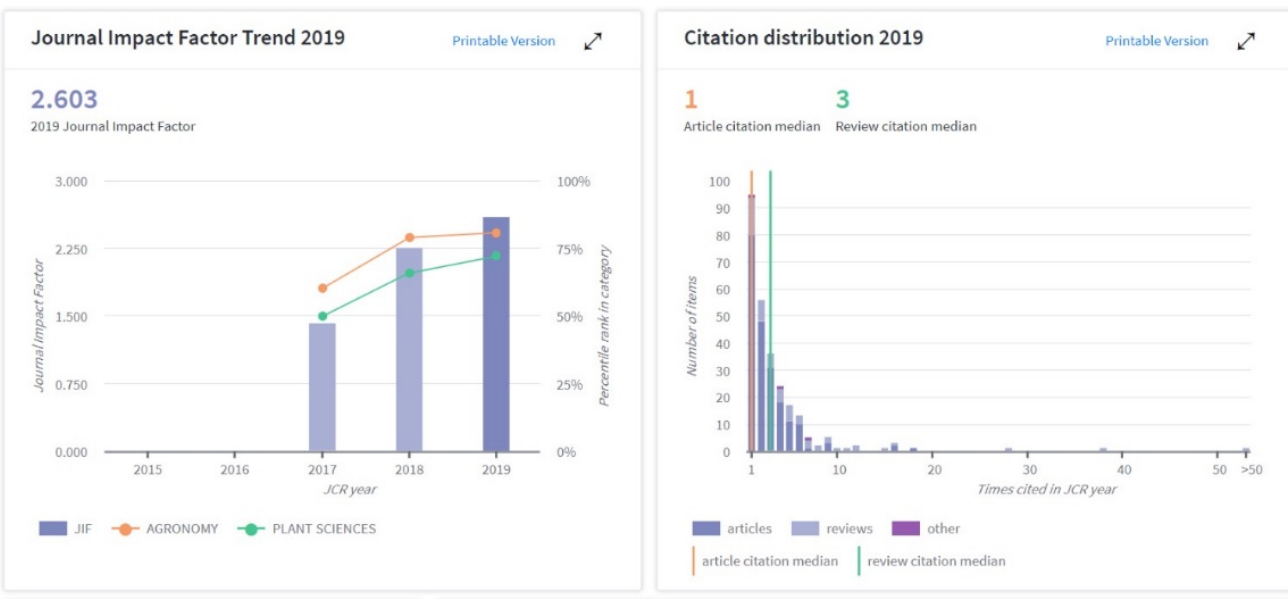

Source data Boxplot Rank Cited Journal Data Citing Journal Data Journal Relationships
Rank

\begin{tabular}{lc}
\hline JCR Impact Factor & $\nearrow$
\end{tabular}

\begin{tabular}{|c|c|c|c|c|c|c|}
\hline \multirow{2}{*}{ JCR Year $\mathbf{-}$} & \multicolumn{3}{|c|}{ AGRONOMY } & \multicolumn{3}{|c|}{ PLANT SCIENCES } \\
\hline & Rank & Quartile & JIF Percentile & Rank & Quartile & JIF Percentile \\
\hline 2019 & $18 / 91$ & Q1 & 80.769 & $65 / 234$ & Q2 & 72.436 \\
\hline 2018 & $19 / 89$ & Q1 & 79.213 & $78 / 228$ & Q2 & 66.009 \\
\hline 2017 & $35 / 87$ & Q2 & 60.345 & $112 / 223$ & Q3 & 50.000 \\
\hline
\end{tabular}

